\title{
Introducing SPHINX-MHD: The Impact of Primordial Magnetic Fields on the First Galaxies, Reionization, and the Global $21 \mathrm{~cm}$ Signal
}

\author{
Harley Katz ${ }^{1 \star}$, Sergio Martin-Alvarez ${ }^{2} \dagger$, Joakim Rosdahl $^{3}$, Taysun Kimm ${ }^{4}$, Jérémy Blaizot ${ }^{3}$, \\ Martin G. Haehnelt ${ }^{2}$, Léo Michel-Dansac ${ }^{3}$, Thibault Garel ${ }^{5}$, Jose Oñorbe ${ }^{6}$, \\ Julien Devriendt ${ }^{1}$, Adrianne Slyz ${ }^{1}$, Omar Attia ${ }^{7,8}$, and Romain Teyssier ${ }^{8}$ \\ ${ }^{1}$ Sub-department of Astrophysics, University of Oxford, Keble Road, Oxford OX1 3RH, UK \\ ${ }^{2}$ Kavli Institute for Cosmology and Institute of Astronomy, Madingley Road, Cambridge CB3 OHA, UK \\ ${ }^{2}$ Univ Lyon, Univ Lyon1, Ens de Lyon, CNRS, Centre de Recherche Astrophysique de Lyon UMR5574, F-69230, Saint-Genis-Laval, France \\ ${ }^{3}$ Department of Astronomy, Yonsei University, 50 Yonsei-ro, Seodaemun-gu, Seoul 03722, Republic of Korea \\ ${ }^{5}$ Observatoire de Geneve, Universite de Geneve, 51 Ch. des Maillettes, 1290 Versoix, Switzerland \\ ${ }^{6}$ Facultad de Fisicas, Universidad de Sevilla, Avda. Reina Mercedes s/n, Campus de Reina Mercedes, 41012 Sevilla, Spain \\ ${ }^{7}$ Department of Physics, ETH Zurich, Wolfgang-Pauli-Strasse 27, CH-8093 Zurich, Switzerland \\ ${ }^{8}$ Institute for Computational Science, University of Zurich, Winterthurerstrasse 190, CH-8057 Zurich, Switzerland
}

Accepted XXX. Received YYY; in original form ZZZ

\begin{abstract}
We present the first results from SPHINX-MHD, a suite of cosmological radiation-magnetohydrodynamics simulations designed to study the impact of primordial magnetic fields (PMFs) on galaxy formation and the evolution of the intergalactic medium (IGM) during the epoch of reionization. The simulations are among the first to employ multi-frequency, on-the-fly radiation transfer and constrained transport ideal MHD in a cosmological context to simultaneously model the inhomogeneous process of reionization as well as the growth of primordial magnetic fields. We run a series of $(5 \mathrm{cMpc})^{3}$ cosmological volumes, varying both the strength of the seed magnetic field and its spectral index. We find that PMFs with a spectral index $\left(n_{B}\right)$ and a comoving amplitude $\left(B_{0}\right)$ that have $n_{B}>-0.562 \log _{10}\left(\frac{B_{0}}{\ln G}\right)-3.35$ produce electron optical depths $\left(\tau_{e}\right)$ that are inconsistent with CMB constraints due to the unrealistically early collapse of low-mass dwarf galaxies. For $n_{B} \geq-2.9$, our constraints are considerably tighter than the $\sim \mathrm{n} G$ constraints from Planck. PMFs that do not satisfy our constraints have little impact on the reionization history or the shape of the UV luminosity function. Likewise, detecting changes in the Ly $\alpha$ forest due to PMFs will be challenging because photoionisation and photoheating efficiently smooth the density field. However, we find that the first absorption feature in the global $21 \mathrm{~cm}$ signal is a particularly sensitive indicator of the properties of the PMFs, even for those that satisfy our $\tau_{e}$ constraint. Furthermore, strong PMFs can marginally increase the escape of LyC photons by up to $25 \%$ and shrink the effective radii of galaxies by $\sim 44 \%$ which could increase the completeness fraction of galaxy surveys. Finally, our simulations show that surveys with a magnitude limit of $\mathrm{M}_{\mathrm{UV}, 1500 \AA}=-13$ can probe the sources that provide the majority of photons for reionization out to $z=12$.
\end{abstract}

Key words: galaxies: high-redshift, (cosmology:) dark ages, reionization, first stars, galaxies: ISM, (ISM:) HII regions, galaxies: star formation

\section{INTRODUCTION}

Understanding the formation of the first generation of galaxies and how the Universe emerged from the Dark Ages remains one of the most interesting frontiers in modern cosmology. During the first billion years, the Universe evolved from a nearly neutral state after recombination to being almost completely ionised. This process of reionization likely began at $z \gtrsim 30$ with the formation of the first metal-free Population III stars (Wise et al. 2012) and ended some- where in the redshift range of $z \sim 5-6$ (Fan et al. 2006; Kulkarni et al. 2019).

Currently, the most commonly discussed scenario is that reionization was primarily driven by photons emitted by dwarf galaxies (e.g., Finkelstein et al. 2019). The number density of these objects combined with their predicted high $\mathrm{LyC}^{1}$ escape fractions $\left(f_{\text {esc }}\right.$, e.g., Kimm et al. 2017) makes them a prime candidate to be the dominant sources of reionization. The majority of these galaxies are too dim to be directly observed, even with our most powerful space telescopes;

\footnotetext{
1 Throughout the paper, we refer to hydrogen-ionising photons with energy
} above $13.6 \mathrm{eV}$ as Lyman continuum photons, or LyC for short.

$\star$ E-mail: harley.katz@ physics.ox.ac.uk
$\dagger$ Co-first author, E-mail: smartin@ast.cam.ac.uk 
however, deep observations of lensed objects behind massive galaxy clusters have hinted at a steep faint-end slope to the UV luminosity function (Livermore et al. 2017). The upcoming launch of the James Webb Space Telescope (JWST) is expected to shed a significant amount of light on the sources of reionization (Gardner et al. 2006).

Much of our detailed knowledge of the physics during the epoch of reionization stems from high-resolution cosmological radiation hydrodynamics simulations. These simulations generally fall into one of two categories: those that model reionization on large (i.e. $\sim 100$ $\mathrm{cMpc}$ ) scales, which are required for an accurate determination of the $21 \mathrm{~cm}$ signal (e.g., Iliev et al. 2014) and to capture the inhomogeneities of reionization relevant for $\mathrm{Ly} \alpha$ forest measurements (Kulkarni et al. 2019), and those that simulate much smaller volumes, focused on galaxy formation and the escape of LyC radiation from a multiphase interstellar medium (ISM) as well as the back-reaction of the formation of the first galaxies and reionization on subsequent galaxy formation (e.g., O'Shea et al. 2015; Rosdahl et al. 2018; Katz et al. 2020b). Due to computational limitations, simulations that model the large-scale inhomogeneous process of reionization on $\sim 100 \mathrm{cMpc}$ scales while simultaneously resolving the escape of ionizing radiation from dwarf galaxies are beyond current capabilities. However, despite the differences between the simulations, they tend to agree that reionization is a highly inhomogeneous process that evolved in an inside-out manner (e.g., Iliev et al. 2006; Lee et al. 2008; Gnedin \& Kaurov 2014; Katz et al. 2017).

Nearly all modern cosmological simulations model their volumes assuming a $\Lambda \mathrm{CDM}$ Universe and a concordance cosmology consistent with that measured from either the Planck (Planck Collaboration et al. 2018) or WMAP (Hinshaw et al. 2013) satellites. The simulations are evolved using the Friedmann equations (Friedmann 1922), and generally explosive feedback is input into the simulation, for example from different types of supernova (SN) or accreting black holes (AGN), in order to explain the Schechter function shape of the $z=0$ stellar mass function (e.g., Vogelsberger et al. 2013; Crain et al. 2015). On-the-fly radiation hydrodynamics has become more common in cosmological simulations (Gnedin \& Abel 2001; Pawlik \& Schaye 2008; Wise \& Abel 2011; Rosdahl et al. 2013; Kannan et al. 2019; Hopkins et al. 2020), but very few include magnetic fields. Magnetic fields are dynamically important in numerous astrophysical contexts such as the ISM (Beck 2007) and the intracluster medium (ICM, Feretti et al. 2012), yet only recently have their effects been self-consistently modelled in cosmological simulations (e.g., Dubois \& Teyssier 2008; Dolag \& Stasyszyn 2009; Doumler \& Knebe 2010; Rieder \& Teyssier 2017; Marinacci et al. 2018; Vazza et al. 2018; Garaldi et al. 2020).

The origin of cosmological magnetic fields is currently unknown and various theories have been proposed to explain their existence. Depending on e.g. the inflationary scenario, primordial magnetic fields (PMFs) could be generated before recombination and magnetic fields of this type will be the primary focus of our work. Current constraints from Planck have placed an upper limit of $\sim 10^{-9} \mathrm{G}$, dependent on the exact structure of the PMF, based on a number of effects PMFs have on CMB anisotropies (Planck Collaboration et al. 2016). Additional constraints on PMFs can be derived from their impact on Big-Bang nucleosynthesis (BBN; Grasso \& Rubinstein 1995; Caprini \& Durrer 2002) and structure formation (Wasserman 1978; Blasi et al. 1999).

During reionization, magnetic fields can also be generated at the edges of ionisation fronts, when electron density and pressure gradients are misaligned (Biermann 1950) or because of charge segregation (Durrive \& Langer 2015; Durrive et al. 2017). They can also be generated during galaxy formation, in or around compact objects such as stars (Beck et al. 2013; Butsky et al. 2017; Martin-Alvarez et al. 2020a) and black holes (Vazza et al. 2017) and expelled into the low density regions of the Universe via magnetised winds. We reserve studying these scenarios for future work. Regardless of their origin, any acceptable magnetogenesis theory must be able to simultaneously explain the order $10^{-6} G$ level magnetic fields observed in galaxies (Davis \& Greenstein 1951; Basu \& Roy 2013) as well as the weak $\left(\gtrsim 10^{-16} G\right)$ magnetic fields in the intergalactic medium (IGM, e.g. Neronov \& Vovk 2010; Dolag et al. 2011; Tavecchio et al. 2011).

Magnetic fields are particularly interesting in the context of reionization for a number of reasons:

(i) Magnetic fields can have a significant impact on the structure and pressure support of the multi-phase ISM (Körtgen et al. 2019). The magnetic energy in the ISM is observed to be in equipartition with the turbulent, thermal and cosmic ray energy densities (Tabatabaei et al. 2008; Beck 2015). Marinacci \& Vogelsberger (2016) demonstrated that star formation histories are not significantly affected, unless the strength of the PMF is $\gtrsim 10^{-9} G$ at which point the additional pressure can suppress gas accretion onto low mass galaxies. However, Martin-Alvarez et al. (2020b) showed that, in the context of ideal MHD, the structure of the ISM and the size of galaxies is noticeably different in the case where seed fields have a strength $<10^{-9} G$, even if the star formation rates remain unchanged as physics associated with the magnetic field can drain angular momentum from the gas and deposit it further away from the centre of the galaxy. As magnetic fields modify the structure of the ISM, they may change $f_{\text {esc }}$, affecting both the history of reionization as well as the sources responsible. If the effective radii of galaxies decrease significantly due to strong PMFs, our interpretations of the highredshift UV luminosity function may also change due to a systematic difference in the size-luminosity relation (Kawamata et al. 2018).

(ii) After recombination, magnetic fields can deposit their energy into the IGM via ambipolar diffusion and decaying MHD turbulence (e.g., Jedamzik et al. 1998; Subramanian \& Barrow 1998; Sethi \& Subramanian 2005). These processes can impact the thermal and ionisation history of the IGM in the early Universe which subsequently translates to a change in the optical depth to the CMB. For sufficiently strong PMFs, these two effects alone can generate electron temperatures of $T_{e}>10^{4} \mathrm{~K}$ and ionise the universe to levels of $\sim 10 \%$ (Sethi \& Subramanian 2005; Chluba et al. 2015). These effects become particularly important for seed fields with $B_{0} \gtrsim 10^{-9} G$ (Sethi \& Subramanian 2005) although this value is approximately equal to the current upper limits on the strength of the PMF (e.g Planck Collaboration et al. 2016).

(iii) Magnetic fields can generate density perturbations, which depending on the strength and spectral slope of the PMF will impact structure formation on small scales with $k$-modes $\gtrsim 1 \mathrm{cMpc}^{-1}$ (Wasserman 1978; Gopal \& Sethi 2003; Shaw \& Lewis 2012). This modification to the matter power spectrum is coincidentally in the region of $k$-space that predominantly affects the formation of dwarf galaxies which, as discussed earlier, are the primary candidates to be the dominant source of reionization. Pandey et al. (2015); Sanati et al. (2020) have shown that the reionization history can significantly change depending on the assumptions regarding PMFs. Hence, the observed reionization history itself provides a constraint on the properties of the PMFs (Sanati et al. 2020). Similarly, the Ly $\alpha$ forest is currently our best constraint on the tail-end of reionization (Fan et al. 2006; Kulkarni et al. 2019) and the effective optical depth is sen- 
sitive to the presence of sub-nG PMFs (e.g., Pandey \& Sethi 2013; Chongchitnan \& Meiksin 2014).

(iv) Although we will not consider them in this work, magnetic fields themselves can be generated at the interfaces of ionisation fronts and density irregularities in the neutral IGM via the Biermann battery (Subramanian et al. 1994) and other mechanisms (e.g. Durrive \& Langer 2015). The Biermann battery will generate magnetic fields as long as there is a gradient in the electron density that is perpendicular to a temperature gradient, for example, in an ionisation front sweeping over a gas filament. Gnedin et al. (2000) post-processed cosmological simulations and found that this mechanism can generate seed magnetic fields on the order of $B \lesssim 10^{-21} G$ in the IGM (see also Attia et al. 2021) and the simulations of Garaldi et al. (2020) show that the Durrive battery generates magnetic fields slightly weaker than those produced by the Biermann battery.

While it is clear that PMFs can significantly impact galaxy formation during the first billion years as well as the reionization history, there are currently no cosmological simulations that systematically study these effects using coupled radiation-magnetohydrodynamics. In this work, we introduce SPHINX-MHD, a suite of simulations that self-consistently model

(i) the formation and evolution of galaxies during the reionization epoch,

(ii) the growth and amplification of primordial magnetic seed fields,

(iii) the escape of ionising radiation from a multi-phase ISM, and

(iv) an inhomogeneous reionization process, using multifrequency radiation transfer and ideal magnetohydrodynamics.

These simulations are an extension of the SPHINX project (Rosdahl et al. 2018; Katz et al. 2020b) which aims to address numerous goals including: understanding the primary sources of reionization, the statistical behaviour of $f_{\text {esc }}$, the back-reaction of radiation feedback on the formation of dwarf galaxies, and the observational signatures of EoR galaxies. SPHINX-MHD goes beyond the goals of the original SPHINX, with the additional aspiration of constraining the impact of magnetic fields on reionization and the formation of the first galaxies. We vary the strength and spectral slope of the PMFs, taking into account their effects on the matter power spectrum (Shaw \& Lewis 2012). When exploring different spectral slopes, our PMF models emulate inflationary magnetogenesis scenarios capable of generating strong magnetic fields (e.g., the models of Turner \& Widrow 1988; Ratra 1992). We depart from an approximately scale-invariant red spectrum case, frequently associated with inflationary magnetogenesis and commonly addressed in the literature (Bonvin et al. 2013; Tasinato 2015; Planck Collaboration et al. 2016). Many of these inflation models allow for a range of spectral indices (Subramanian 2010 , 2016) such that there is no back reaction on the expansion during inflation. Furthermore the amplitude of the magnetic field is extremely sensitive to parameters chosen for the inflation model and depends as well on coupling function between the inflaton and the electromagnetic field.

This paper is organised as follows. In Section 2, we describe the numerical methods including initial condition generation, simulation physics, and halo finding. In Section 3, we present our results on the impact of magnetic fields on reionization and galaxy formation during the first billion years. Finally, in Section 4, we present our discussion and conclusions.

\section{NUMERICAL METHODS}

In total, we run six simulations as listed in Table 1, varying the strength of the primordial seed magnetic field and its spectral slope, accounting for its effect on the matter power spectrum (Shaw \& Lewis 2012). These simulations are designed to explore a representative subset of the parameter space for which magnetic fields may be interesting in the context of reionization. The lowest magnetic field strength is approximately that of a Biermann battery-generated magnetic field. The B14 simulation is used for comparison to other simulations in the literature that explored primordial seed fields of this value (e.g., Pillepich et al. 2018). Finally the B11 simulations represent scenarios where the magnetic field is likely to have a dynamic impact on galaxy structure (e.g., Martin-Alvarez et al. 2020b), and where the impact of the primordial seed field on the matter power spectrum can modify structure formation and potentially the reionization history (Shaw \& Lewis 2012; Pandey et al. 2015; Sanati et al. 2020). We describe below in detail the numerical methods for each of these simulations.

\subsection{Initial Conditions}

Initial conditions for the simulations are generated with MUSIC (Hahn $\&$ Abel 2011) at $z=150$ assuming a $\Lambda$ CDM Universe with the following cosmological parameters: $\Omega_{\Lambda}=0.6825, \Omega_{\mathrm{m}}=0.3175$, $\Omega_{\mathrm{b}}=0.049, h=0.6711, \sigma_{8}=0.83$, and $n_{s}=0.962$, consistent with the Planck 2013 results (Planck Collaboration et al. 2014). We set the initial gas composition of the simulation to be $76 \% \mathrm{H}$ and $24 \% \mathrm{He}$ by mass and assume a metallicity floor of $3.2 \times 10^{-4} Z_{\odot}$ to account for the lack of cooling due to molecular hydrogen and associated Pop. III star formation in our simulation (Wise et al. 2012).

The initial conditions represent a comoving cubic volume with a side length of $5 \mathrm{cMpc}^{2}$, initially populated with $512^{3}$ dark matter particles of mass $3.1 \times 10^{4} \mathrm{M}_{\odot}$ and the same number of gas cells. Assuming that we require 300 dark matter particles to resolve a halo, the minimum halo mass resolved by our simulation is $9.3 \times$ $10^{6} \mathrm{M}_{\odot}$, which is well below the atomic cooling threshold. Because the simulated volume is considerably smaller than the cosmological homogeneity scale of $\sim 100 \mathrm{cMpc}$, the volume was selected among 60 different dark matter-only simulations for having the most average halo mass function at $z=6, z=8$, and $z=10$ (see Rosdahl et al. 2018).

The magnetic fields in the simulations are initialised in two different ways. The B21, B14, and B11 simulations exhibit uniform seed fields that are aligned with the $z$-axis of the simulation. The comoving strengths of the seed fields for these simulations are listed in Table 1. The transfer function used to compute the initial conditions for these simulations is calculated from the fitting function presented in Eisenstein \& $\mathrm{Hu}$ (1998) and the initial density field is identical between these different models. The seed fields in the B11_29, B11_27, and B11_24, are initialised to have a power spectrum that is described as:

$P(k)=A k^{n_{B}}$,

where $n_{B}$ is the spectral slope and

$A=\frac{(2 \pi)^{n_{B}+5} B_{1 \mathrm{cMpc}}^{2}}{2 \Gamma\left(\frac{n_{B}+3}{2}\right) k_{1 \mathrm{cMpc}}^{n_{B}+3}}$.

2 Note that all length units that are prefaced with a "c" represent comoving units while all others are physical. 
Table 1. List of the simulations in the SPHINX-MHD suite along with details of each run. From left to right, columns indicate the name of the simulation, the comoving strength of the initial magnetic field $\left(B_{0}\right.$; see text for details), the spectral index of the magnetic field, the dark matter $\left(M_{\mathrm{DM}}\right)$ and stellar $\left(M_{*}\right)$ particle mass resolution, the cell sizes at $z=6\left(\Delta x_{z=6}\right)$ and $z=20\left(\Delta x_{z=20}\right)$, the final redshift of the simulation, and the initial structure of the magnetic field. In each simulation, we only change the initial conditions for magnetic fields varying both their strength and spectral index $n_{B}$.

\begin{tabular}{lcccccccc}
\hline Simulation Name & $B_{0}[G]$ & $n_{B}$ & $M_{\mathrm{DM}}\left[\mathrm{M}_{\odot}\right]$ & $M_{*}\left[\mathrm{M}_{\odot}\right]$ & $\Delta x_{z=6}[\mathrm{pc}]$ & $\Delta x_{z=20}[\mathrm{pc}]$ & $z_{\text {final }}$ & Seed Field Structure \\
\hline B21 & $10^{-21}$ & - & $3.1 \times 10^{4}$ & $10^{3}$ & $7.31 h^{-1}$ & $2.44 h^{-1}$ & 6.0 & Uniform, $z$-direction \\
B14 & $10^{-14}$ & - & $3.1 \times 10^{4}$ & $10^{3}$ & $7.31 h^{-1}$ & $2.44 h^{-1}$ & 6.0 & Uniform, $z$-direction \\
B11 & $5 \times 10^{-11}$ & - & $3.1 \times 10^{4}$ & $10^{3}$ & $7.31 h^{-1}$ & $2.44 h^{-1}$ & 6.0 & Uniform, $z$-direction \\
B11_29 & $5 \times 10^{-11}$ & -2.9 & $3.1 \times 10^{4}$ & $10^{3}$ & $7.31 h^{-1}$ & $2.44 h^{-1}$ & 6.0 & Random \\
B11_27 & $5 \times 10^{-11}$ & -2.7 & $3.1 \times 10^{4}$ & $10^{3}$ & $7.31 h^{-1}$ & $2.44 h^{-1}$ & 6.0 & Random \\
B11_24 & $5 \times 10^{-11}$ & -2.4 & $3.1 \times 10^{4}$ & $10^{3}$ & $7.31 h^{-1}$ & $2.44 h^{-1}$ & 54.6 & Random \\
\hline
\end{tabular}

In this initial configuration, we follow the convention to normalise the strength of the magnetic field at a scale of $1 \mathrm{cMpc}\left(B_{0}=B_{\lambda=1 \mathrm{cMpc}}\right.$, e.g. Shaw \& Lewis 2012; Planck Collaboration et al. 2016). Our method for generating the magnetic component of the initial conditions for the B11_29, B11_27, and B11_24 simulations will be further described in Martin-Alvarez et al. in prep). In short, to generate these ICs, we initialise a random Gaussian vector potential field in Fourier space over a uniform grid. We modulate this spectrum at each wavelength $k$ so that the magnetic field resulting from the curl of the vector potential has a spectral slope $n_{B}$. The computed magnetic field is divergenceless by construction and spatially-displaced in Fourier space so that it is defined at cell interfaces. Finally, the norm of the magnetic power spectrum is set using Equation 2. The resulting magnetic field perturbations deviate from the average field with a magnetic field rms $\sigma_{\mathrm{rms}, B} /\langle B\rangle$ of $\sim 1.25$ for each set of ICs.

For the B11_29, B11_27, and B11_24 simulations, we also account for the impact that the PMFs have on the matter power spectrum. In order to account for the impact of the PMF on the matter power spectrum, we use a modified version of CAMB (Lewis et al. 2000; Shaw \& Lewis 2012) to compute the transfer function used by MUSIC. The general idea is that magnetic fields generate density perturbations in the baryons via the Lorentz force (Wasserman 1978; Kim et al. 1996; Subramanian \& Barrow 1998; Gopal \& Sethi 2003; Sethi \& Subramanian 2005; Tashiro \& Sugiyama 2006). These magneticallyinduced perturbations grow at the same rate as the primordial density fluctuations and couple to the dark matter via gravity. For strong enough PMFs, the additional density perturbations can dominate over primordial fluctuations, especially at high $k$. Hence, the initial density field is different in the simulations with non-uniform magnetic fields compared to the B21, B14, and B11 simulations.

For our chosen cosmology, the transfer function computed with CAMB deviates from that of Eisenstein \& Hu (1998) at both high and low $k$-modes by up to $\sim 20 \%$ in the case without PMFs (see Figure 1). We have confirmed that despite these differences, the impact on the simulation is negligible as there are no noticeable differences in the dark matter halo mass function at $z=6$ (see the dashed and purple lines in the bottom panel of Figure 2). Note that our limited simulation volume inhibits us from testing changes at $k \lesssim 0$. 2 . In contrast, when we include the scalar and tensor perturbations from PMFs, the more the spectral slope of the PMF deviates from -3 (i.e. scale-free), the larger the differences in the matter power spectrum compared to standard $\Lambda \mathrm{CDM}$.

In Figure 2, we plot the matter power spectrum for different values of $n_{B}$ for $B_{0}=0.05 \mathrm{nG}$. The enhancements in the matter power spectra at high $k$ due to the PMFs have a significant impact on the dwarf halo population at $z=6$ (see the bottom panel of Figure 2). Depending on the spectral slope, an excess of more than an order of magnitude in the number of haloes is seen at certain masses

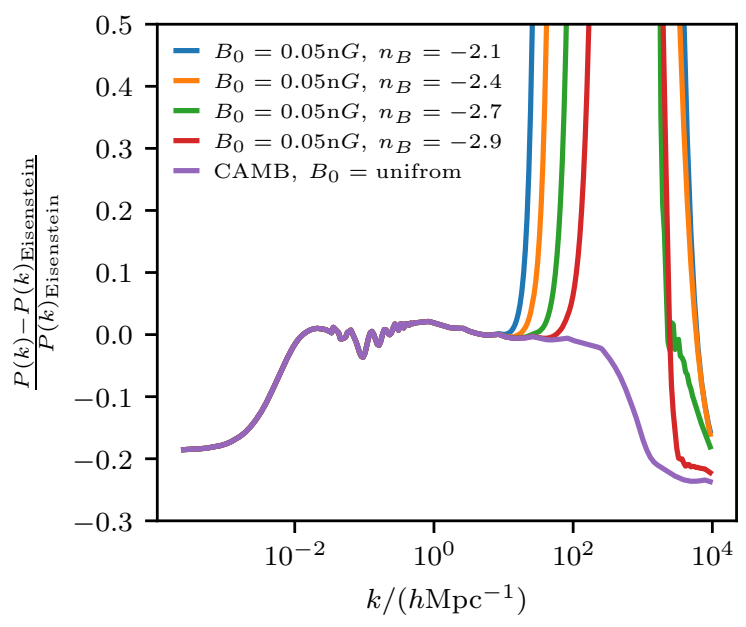

Figure 1. Fractional difference between the input matter power spectra for simulations that use CAMB (with no effects due to $B_{0}$ ) or the modified version of CAMB that accounts for PMFs when compared with those of the fiducial model that employ the fitting function from Eisenstein \& Hu (1998). In the standard $\Lambda \mathrm{CDM}$ case, the results differ by up to $\sim 20 \%$ at high and low $k$ while the differences due to the PMFs completely dominate any systematic error due to differences in transfer function.

compared with $\Lambda \mathrm{CDM}$. These are clearly visible in Figure 3 where we show the dark matter column density viewed down the $z$-axis of the simulation box at $z=6$ for the set of dark matter-only simulations. For $n_{B}=-2.1$, much of the filamentary structure is affected. Because the amount of mass in the box is conserved, the increase in the number of dwarf galaxies results in a small reduction at the high-mass end. For PMFs with significantly lower amplitudes, reasonable choices for $n_{B}$ do not lead to any significant changes in the matter power spectrum; hence, we only adopt these modified initial conditions for different realisations of the B11 simulation.

\subsection{Gravity, Magnetohydrodynamics, \& Radiation}

In order to evolve the simulation with gravity, magnetohydrodynamics, and radiation transfer (RT), we use RAMSES-RT (Rosdahl et al. 2013; Rosdahl \& Teyssier 2015), which is a radiation hydrodynamics extension of the RAMSES code (Teyssier 2002). The public version of the RAMSES code includes a constrained transport (CT, Evans \& Hawley 1988) implementation of ideal MHD (Teyssier et al. 2006; Fromang et al. 2006). For this work, we have updated the version of the code used for the original SPHINX simulations (Rosdahl et al. 

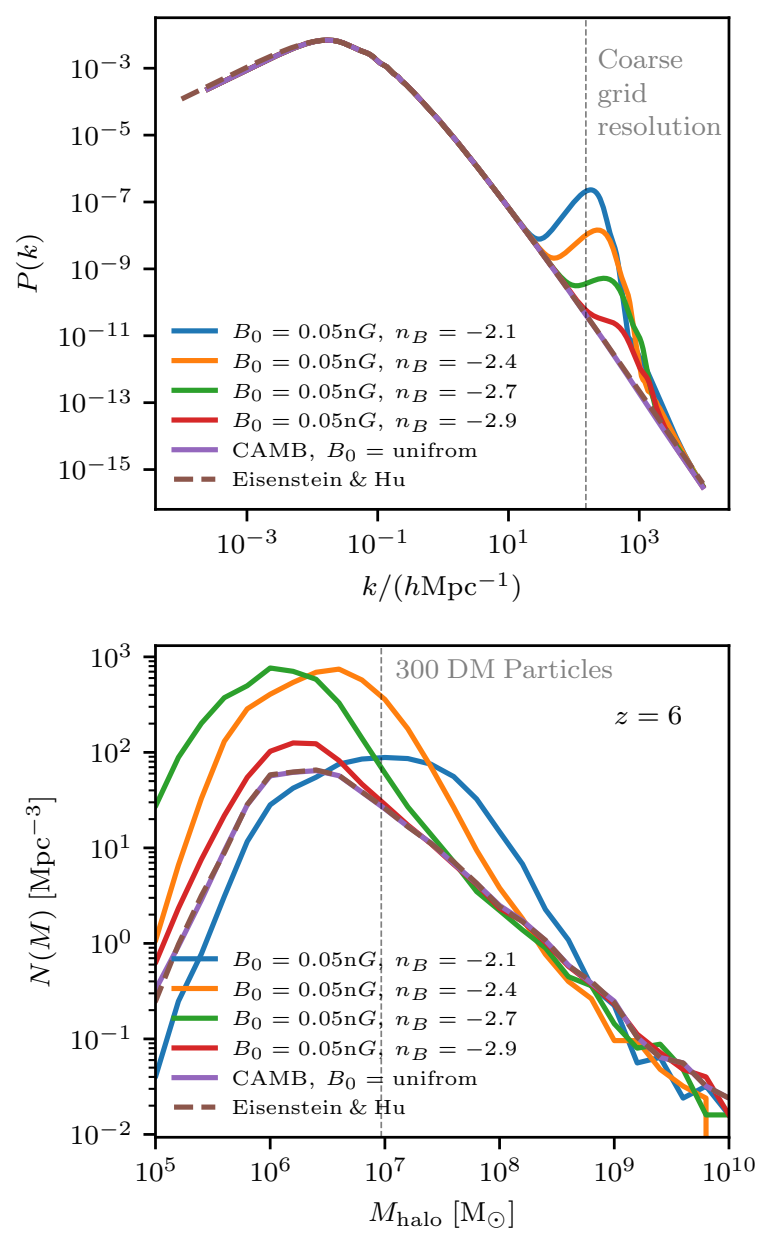

Figure 2. Input matter power spectra (top) and dark matter halo mass function at $z=6$ for the various sets of initial conditions computed as the number of halos in mass bins of width 0.2 dex (bottom) for dark matter-only simulations. The CAMB and Eisenstein \& Hu (1998) models do not include any magnetic field effects. The enhancements in the matter power spectra due to PMFs can significantly alter the dark matter halo mass function at $z=6$ depending on the spectral slope of the PMF. These differences occur in the range of masses represented by dwarf galaxies. Despite the $\sim 20 \%$ differences between the CAMB and Eisenstein \& Hu (1998) at high and low $k$, no significant differences arise in the halo mass function at $z=6$. The turnover in the mass function at low halo masses is due to limited resolution. We show all haloes that are resolved by more than 20 particles.

2018) so that it can simultaneously solve the equations for ideal radiation-magnetohydrodynamics (RMHD).

\subsubsection{Gravity and Hydrodynamics}

The ideal MHD equations are solved using a second-order Godunov scheme based on a MUSCL-Hancock method. In contrast to the original SPHINX simulation, we employ the HLLD Riemann solver (Miyoshi \& Kusano 2005) and the MinMod slope limiter (Roe 1986) to construct gas variables at cell interfaces from their cell-centred values. We assume an adiabatic index of $\gamma=5 / 3$ (i.e. that of an ideal monatomic gas) to close the relation between gas pressure and internal energy. The motions of collisionless dark matter, star particles and gas are computed by solving the Poisson equation. Dark matter and star particles are projected onto the adaptive grid using

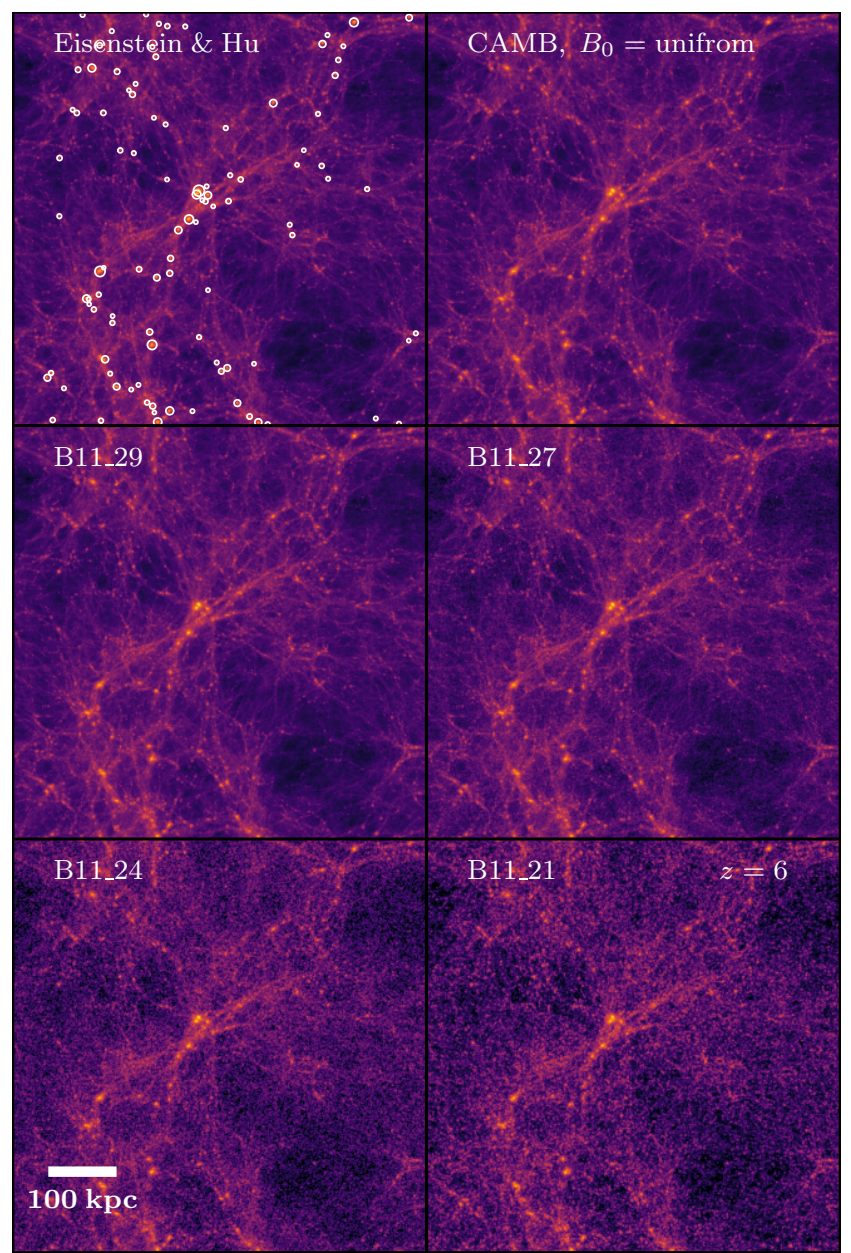

Figure 3. Dark matter column density maps of all of the different initial conditions at $z=6$ in the dark matter-only simulations. The CAMB and Eisenstein \& Hu (1998) models do not include any magnetic field effects. As the slope of the PMF power spectrum flattens, more low mass dark matter haloes appear, impacting the filamentary structure of the cosmic web. The locations of the 100 most massive haloes are shown in the top left with each circle representing the virial radius of the halo.

a cloud-in-cell interpolation. A multigrid solver (Guillet \& Teyssier 2011) is used to solve the Poisson equation up to a refinement level of 12 . At more refined levels we adopt a conjugate gradient solver to improve the speed of the simulation.

\subsubsection{Radiative Transfer}

Radiation is advected between cells using a first-order moment method that uses the M1 closure for the Eddington tensor (Levermore 1984) and a Global-Lax-Friedrich intercell flux function (e.g., Toro 2009). In comparison to many other RT solvers, the M1 closure relies only on local quantities and does not scale with the number of radiation sources in the computational volume. Because of our choice of RT solver, the time steps in our simulations are limited by the RT Courant condition such that $\Delta t<\Delta x / 3 c_{\text {sim }}$, where $\Delta x$ is the size of a cell, and $c_{\text {sim }}$ is the speed of light chosen for the simulation. Setting $c_{\text {sim }}=c$ would result in a prohibitively small time step that in some cases is $\sim 1000 \times$ smaller than that of a simulation without RT. For this reason, we adopt the variable-speed-of-light approximation (VSLA) described in Katz et al. (2017) where we adaptively change 
$c_{\text {sim }}$ depending on the local grid refinement level so that the speed of ionisation fronts is properly captured in both low- and high-density regions. We adopt a value of $c_{\text {sim }}=0.2 c$ on the base (coarse) grid of the simulation and divide this quantity by a factor of two on each subsequently refined level. We set a minimum $c_{\text {sim }}=0.0125 c$ to ensure that the radiation velocity is always greater than that of the gas. Compared to the original implementation of VSLA in Katz et al. (2017), we employ an updated and much more computationally efficient version of the algorithm (Katz et al. 2018; Rosdahl et al. 2018) that is integrated with the adaptive time stepping on the AMR grid as well as the RT subcycling scheme present in RAMSES-RT (Rosdahl et al. 2018). To further reduce the computational demands of the RT, we allow for up to 500 RT subcycles for each hydrodynamic time step by adopting Dirichlet boundary conditions at coarse-fine interfaces (Commerçon et al. 2014). In practice, the actual number of RT subcycles is of $O(<10)$ after the first $\mathrm{SNe}$ explode in the simulation.

The radiation in the simulation is tracked in three energy bins: $13.6 \mathrm{eV}-24.59 \mathrm{eV}, 24.59 \mathrm{eV}-52.54 \mathrm{eV}$, \& $52.54 \mathrm{eV}-\infty$. This allows us to track the ionisation states of hydrogen and helium. The mean energy of the radiation within each frequency bin is computed every ten coarse time steps as the luminosity-weighted mean energy across all star particles in the simulation. The number of photons in each frequency bin for each cell is updated on every fine time step and we apply the "smoothing" technique to reduce the total number of cooling subcycles (Rosdahl et al. 2013).

\subsubsection{Ideal MHD}

As stated earlier, the simulation includes an implementation of ideal MHD using a CT method. Unlike the other hydrodynamic quantities in the simulation whose properties are stored as cell-centred quantities, the induction equation is solved in an integral form that requires the magnetic field properties to be stored on the faces of each cell in the AMR grid (Teyssier et al. 2006; Fromang et al. 2006). This consists of storing six $B$-field quantities for each gas cell. The CT method allows us to maintain the solenoidal constraint and conserve $\nabla \cdot B=0$ to machine precision, in contrast to divergence cleaning methods (e.g., Powell et al. 1999; Dedner et al. 2002). These divergence cleaning methods often struggle in certain astrophysical situations which may cause artificial amplification of the magnetic field (e.g., Hopkins \& Raives 2016) and may no longer conserve physical quantities (Tóth 2000). Our code uses a divergence-preserving scheme to interpolate the magnetic field at coarse-fine boundaries of the grid (Balsara 2001; Tóth \& Roe 2002). To demonstrate the divergence-less behaviour, in Figure 4 we plot the maximum (solid) and average (dashed) divergence with respect to the maximum value of the local $B$-field $\left(|\vec{\nabla} \cdot \vec{B}| \Delta x /\left|B_{\text {loc }}\right|\right)$ as a function of density at $z=6$ for different simulations. For the simulations that are initialised with a uniform magnetic field along the $z$-axis, the maximum divergence never becomes greater than $1 \%$ of the maximum $B$-field on the face of any cell, indicating that it has little impact on the dynamics in our simulation. For the simulations initialised with a random magnetic field with a given spectral slope and normalisation, the divergence is significantly greater due to the simulations being initialised using single precision. However, even for these simulations, the divergence errors are not dynamically important. Inside of galaxies, the divergence of the magnetic field is often six to ten orders of magnitude below the strength of the local $B$-field, demonstrating the near-machine precision conservation of the solenoidal constraint provided by the CT algorithm.

In contrast to simulations without magnetic fields, the time step in our simulations has an additional constraint where it can be limited

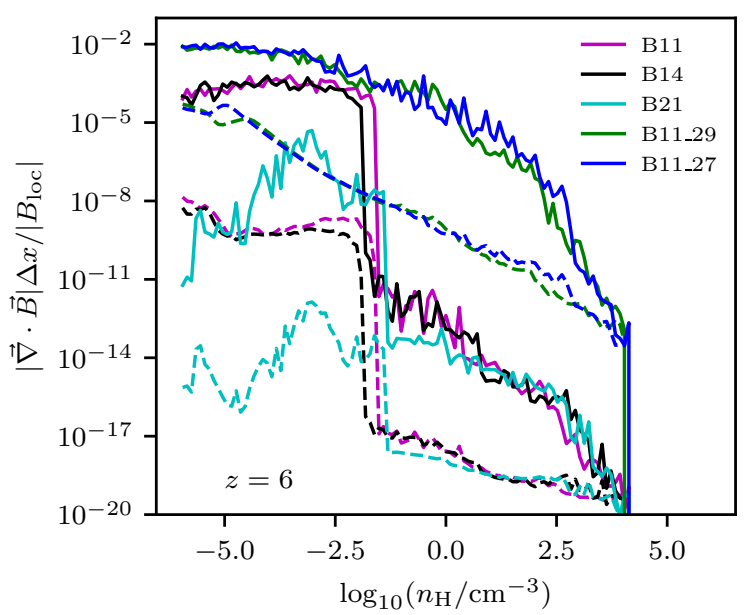

Figure 4. Maximum (solid) and average (dashed) divergence with respect to the maximum value of the local $B$-field $\left(|\vec{\nabla} \cdot \vec{B}| \Delta x /\left|B_{\text {loc }}\right|\right)$ as a function of density at $z=6$ for different simulations. The maximum divergence never becomes greater than $1 \%$ of the maximum of the local $B$-field, indicating that it has little impact on the dynamics in our simulation. The sharp feature that occurs in the B11, B14, and B21 simulations at $n_{\mathrm{H}} \sim 10^{-1.5} \mathrm{~cm}^{-3}$ is due to $\mathrm{SN}$ feedback as it only occurs for $T>10^{5} \mathrm{~K}$ gas. This feature is erased in the B11_27 and B11_29 simulations where the divergence is already higher than the values in the other simulation after the jump.

by the Alfvén velocity $\left(v_{A}\right)$, where

$v_{A}=\frac{B}{\sqrt{\mu_{0} \rho}}$,

where $\rho$ is the gas density and $\mu_{0}$ is the magnetic permeability of vacuum. In general, the Alfvén velocity does not set the strongest limit on the simulation time step, except when the magnetic field strength is very high and the density is low. This situation can occur after $\mathrm{SN}$ events when the density drops considerably in the case of strong PMFs (e.g. the B11 simulation). In these regions, the additional magnetic pressure is extremely efficient in reducing the density of the SN bubbles causing the time step to drop to values of order years. To avoid this issue, we force a density floor of $3 \times 10^{-9} \mathrm{H} \mathrm{cm}^{-3}$ in regions with $B>5 \times 10^{-8} G$. The value we choose for the density floor is considerably lower than the density reached in any of the simulations that never satisfy this condition; hence the floor does not impact the reionization of the IGM. Furthermore, the density floor does not impact the escape of ionising photons from galaxies as the conditions for the floor are only met in regions of strong SN feedback where the gas is very far into the optically thin regime.

Although ideal MHD is the current state-of-the-art for large cosmological simulations, it is well established that non-ideal effects can impact both the dynamics and thermodynamical state of the ISM (e.g., Machida et al. 2008; Duffin \& Pudritz 2009; Marchand et al. 2018) as well as the state of the IGM post recombination (e.g. Sethi $\&$ Subramanian 2005). For the simulations presented here, the scales that we resolve are significantly larger than those required for individual star formation where non-ideal effects are important. However, for strong enough magnetic fields, on large scales, non-ideal effects may be important since ambipolar diffusion and decaying magnetic turbulence can both ionise and significantly increase the electron temperature $\left(T_{e}\right)$ of the IGM (e.g., Jedamzik et al. 1998; Subramanian \& Barrow 1998; Sethi \& Subramanian 2005). Using recfast++ (Chluba et al. 2015), in Figure 5, we plot the ionised fraction $Q_{\mathrm{HII}}$ and $T_{e}$ of the IGM as a function of redshift for various initial values 

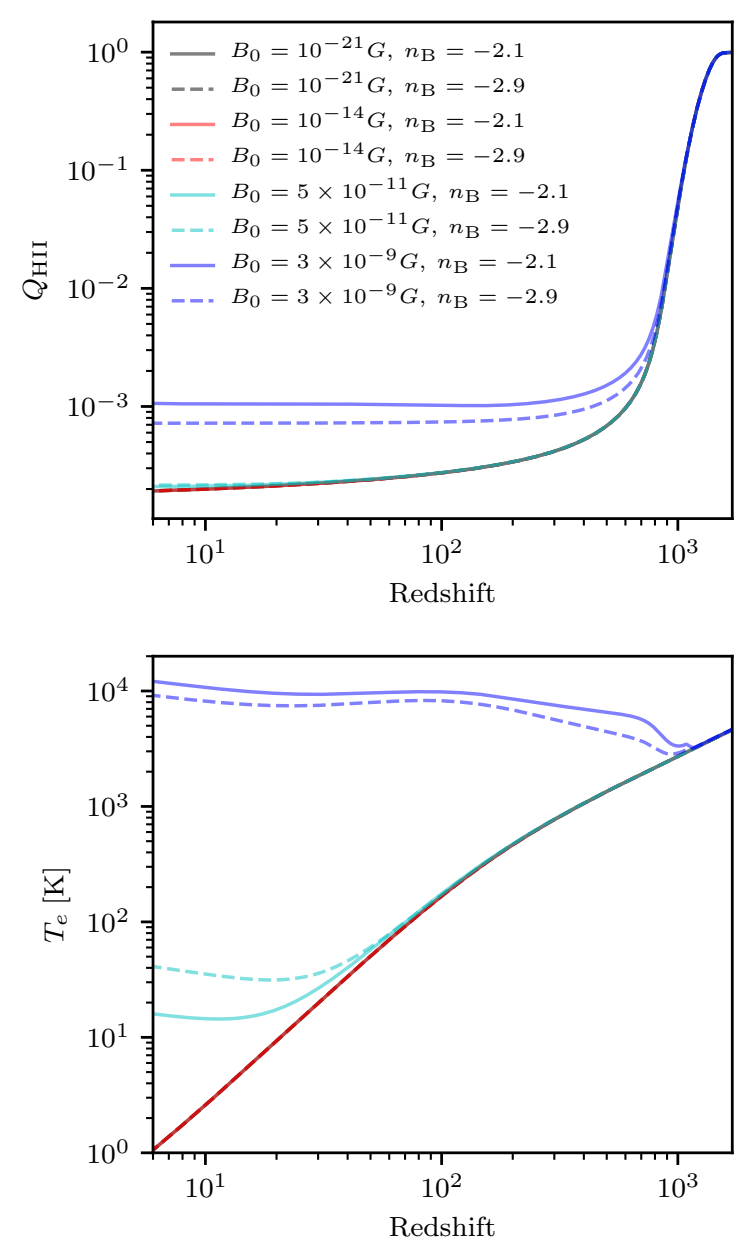

Figure 5. Hydrogen ionisation fraction (top) and electron temperature (bottom) as a function of redshift for a variety of seed field strengths $\left(B_{0}\right)$ and spectral indices $\left(n_{\mathrm{B}}\right)$ as a result of ambipolar diffusion and decaying magnetic turbulence. These calculations were made with recfast++ (Chluba et al. 2015). The ionisation fraction is only impacted for seed fields that are much stronger than those used in this work. Similarly the impact of both ambipolar diffusion and decaying magnetic turbulence is very unlikely to have a serious impact on our results as the temperature change is $\ll 100 \mathrm{~K}$.

of $B_{0}$ and $n_{B}$, in the absence of other ionising sources. For seed fields with $B_{0}>1 \mathrm{n} G$, a significant amount of heating and residual ionisation occurs. In contrast, for the values of the seed fields considered in the this work (see Table 1), both effects will have limited impact on the simulation; hence we can exclude them. There is a small temperature enhancement in the IGM for the scenario with $B_{0}=0.05 \mathrm{n} G$ at $z<40$; however, this is much below the ionisation temperature of hydrogen and is unlikely to impact our results.

\subsubsection{Cooling \& Non-Equilibrium Chemistry}

Gas cooling and non-equilibrium chemistry in the SPHINX-MHD simulations follow very closely to the prescriptions used in the original SPHINX simulations. We employ a six species non-equilibrium chemistry model that tracks $e$, HI, HII, HeII, \& HeII, which are fully coupled to the radiation transfer through photoionization, photoheating, and UV radiation pressure. For these primordial species, we compute cooling and heating due to photoionization, collisional ionisation, collisional excitation, recombination, bremsstrahlung, Comp- ton cooling/heating off the cosmic-microwave background, and dielectronic recombination (Rosdahl et al. 2013). In addition, we compute cooling due to metal lines. At $T>10^{4} \mathrm{~K}$, cooling from metals is computed by interpolating cooling tables generated with CLOUDY (Ferland et al. 1998) that were calculated assuming photoionization equilibrium with a UV background (Haardt \& Madau 1996). At $T<10^{4} \mathrm{~K}$, we use the fine structure cooling rates from Rosen \& Bregman (1995). A density- and redshift-independent temperature floor of $15 \mathrm{~K}$ is used throughout the simulation volume.

\subsection{Refinement}

Taking advantage of the adaptive grid in RAMSES, we allow the gas cells to refine when certain criteria are fulfilled. A cell is refined into eight equal-volume children cells using a quasi-Lagrangian scheme if its contained dark matter mass summed with its stellar and gas mass multiplied by $\Omega_{\mathrm{m}} / \Omega_{\mathrm{b}}$ is greater than eight times the dark matter particle mass. Furthermore, we also allow a cell to refine if the width of the cell is larger than $25 \%$ of the local Jeans length. We allow for up to 16 total levels of refinement which results in a spatial resolution of $7.31 h^{-1} \mathrm{pc}$ at $z=6$. As in the original SPHINX simulation, rather than maintain an approximately constant physical resolution by releasing new AMR levels at predefined redshifts, we allow the simulation to refine to level 16 at any redshift. This implies that the physical cell width is significantly smaller at higher redshifts (e.g,. $\Delta x=$ $2.44 h^{-1}$ pc at $z=20$ ). In order to prevent particle scatterings due to strong two-body interactions, we smooth the dark matter particle density on one refinement level coarser than the maximum.

\subsection{Star Formation}

Collisionless star particles are allowed to form only in gas cells at the maximum level of refinement depending on the local properties of the gas. We employ a magneto-thermo-turbulent (MTT) star formation criteria (Padoan \& Nordlund 2011; Hennebelle \& Chabrier 2011; Federrath \& Klessen 2012). We define the MTT Jeans length as:

$\lambda_{\mathrm{J}, \mathrm{MTT}}=\frac{\pi \sigma_{V}^{2}+\sqrt{36 \pi c_{\mathrm{s}, \mathrm{eff}}^{2} G \Delta x^{2} \rho+\pi \sigma_{V}^{4}}}{6 G \rho \Delta x}$,

where $G$ is the gravitational constant and $\sigma_{V}$ is the gas turbulent velocity. The effective sound speed, $c_{\mathrm{s} \text {,eff }}$, accounts for small-scale pressure support due to the presence of magnetic fields such that

$c_{\mathrm{s}, \mathrm{eff}}=c_{\mathrm{s}} \sqrt{1+\beta^{-1}}$,

where $\beta=P_{\text {thermal }} / P_{\text {mag }}{ }^{3}$. Star particles are only allowed to form when the local gas density is $>10 \mathrm{~cm}^{-3}$, the cell is a local density maximum compared to its immediate neighbours, the gas velocities are locally convergent, and the MTT Jeans length is unresolved such that $\Delta x>\lambda_{\mathrm{J}, \mathrm{MTT}}$. When a gas cell satisfies these criteria, stars are formed following a Schmidt law (Schmidt 1959) with a star formation rate of

$\dot{\rho}_{\mathrm{star}}=\epsilon_{\mathrm{ff}} \frac{\rho}{t_{\mathrm{ff}}}$.

3 Typical value for $\beta$ in star-forming gas in the B14 and B21 simulations are $10^{5}$ and $10^{19}$, respectively and hence the magnetic field does not impact the effective sound speed in these simulations. For the B11 simulation, $\beta$ can drop to values $\ll 1$ for very cold gas, substantially increasing the effective sound speed. 


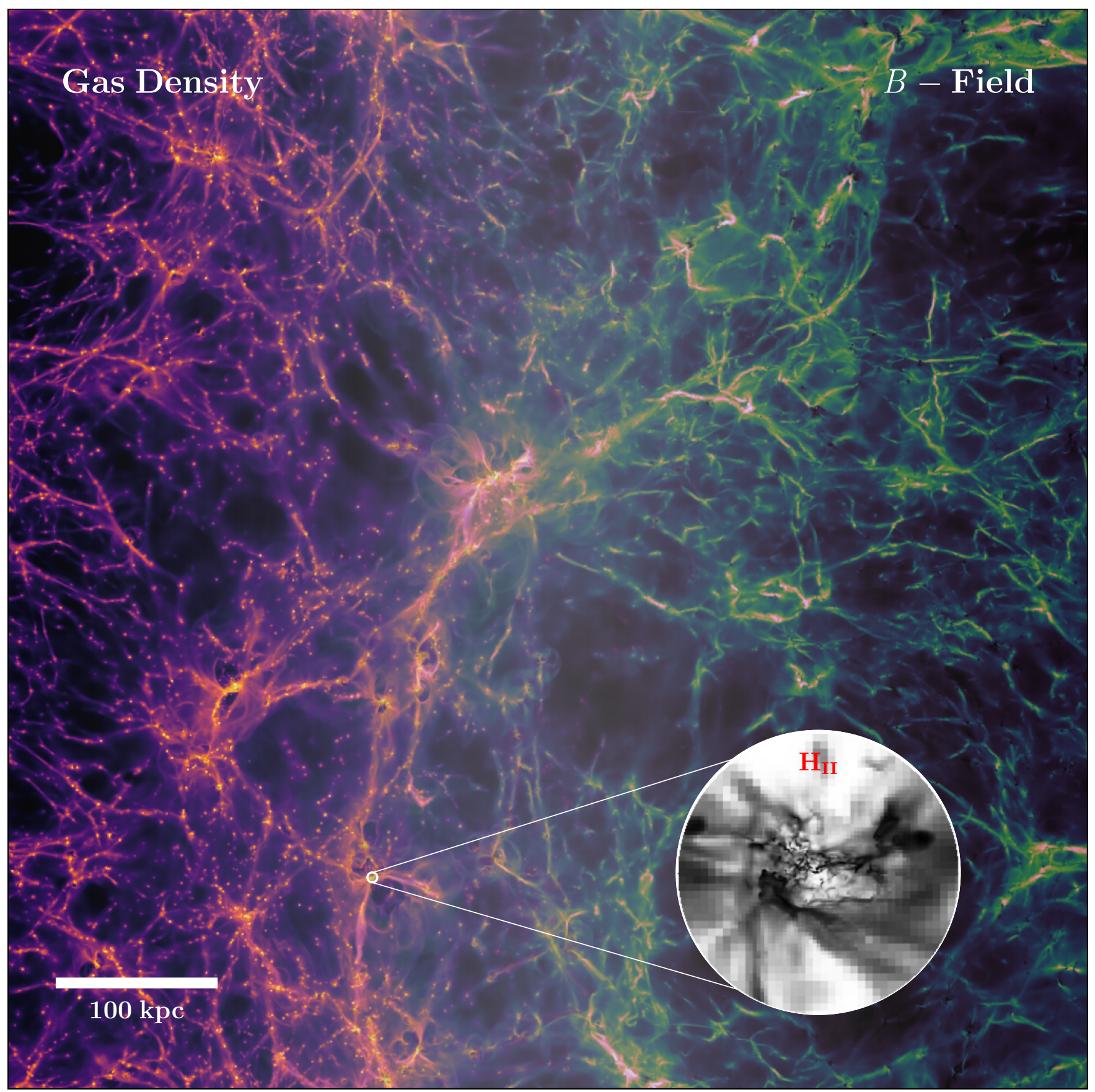

Figure 6. Average gas density (left) and magnetic energy density (right) along the line-of-sight for the B14 simulation at $z=6$. The structure of the magnetic field follows that of the cosmic web. To illustrate the dynamic range of the simulations, the inset shows a map of the HII fraction for an individual galaxy where black represents neutral regions and white represents ionised regions.

The free-fall time of the gas is defined as

$t_{\mathrm{ff}}=\sqrt{\frac{3 \pi}{32 G \rho}}$.

The local efficiency of star formation, $\epsilon_{\mathrm{ff}}$, is computed from the magneto-thermodynamical properties of the host gas cell following the multi-scale PN model (Padoan \& Nordlund 2011) from Federrath
\& Klessen (2012) such that:

$\epsilon_{\mathrm{ff}}=\frac{\epsilon_{\mathrm{cts}}}{2 \phi_{t}} \exp \left(\frac{3}{8} \sigma_{s}^{2}\right)\left[1+\operatorname{erf}\left(\frac{\sigma_{s}^{2}-s_{\mathrm{crit}}}{\sqrt{2 \sigma_{s}^{2}}}\right)\right]$.

Here, $\sigma_{s}=\ln \left(\rho_{\text {gas }} /\left\langle\rho_{\text {gas }}\right\rangle\right), 1 / \phi_{t}=0.57$ takes into account the uncertainty in free-fall timescales at the mean density of the cloud and that of higher density gas, and $\epsilon_{\mathrm{cts}}=0.5$ represents the maximum fraction of the gas that can be converted into stars when accounting for proto-stellar feedback. Finally, $s_{\text {crit }}$ is the critical density beyond 


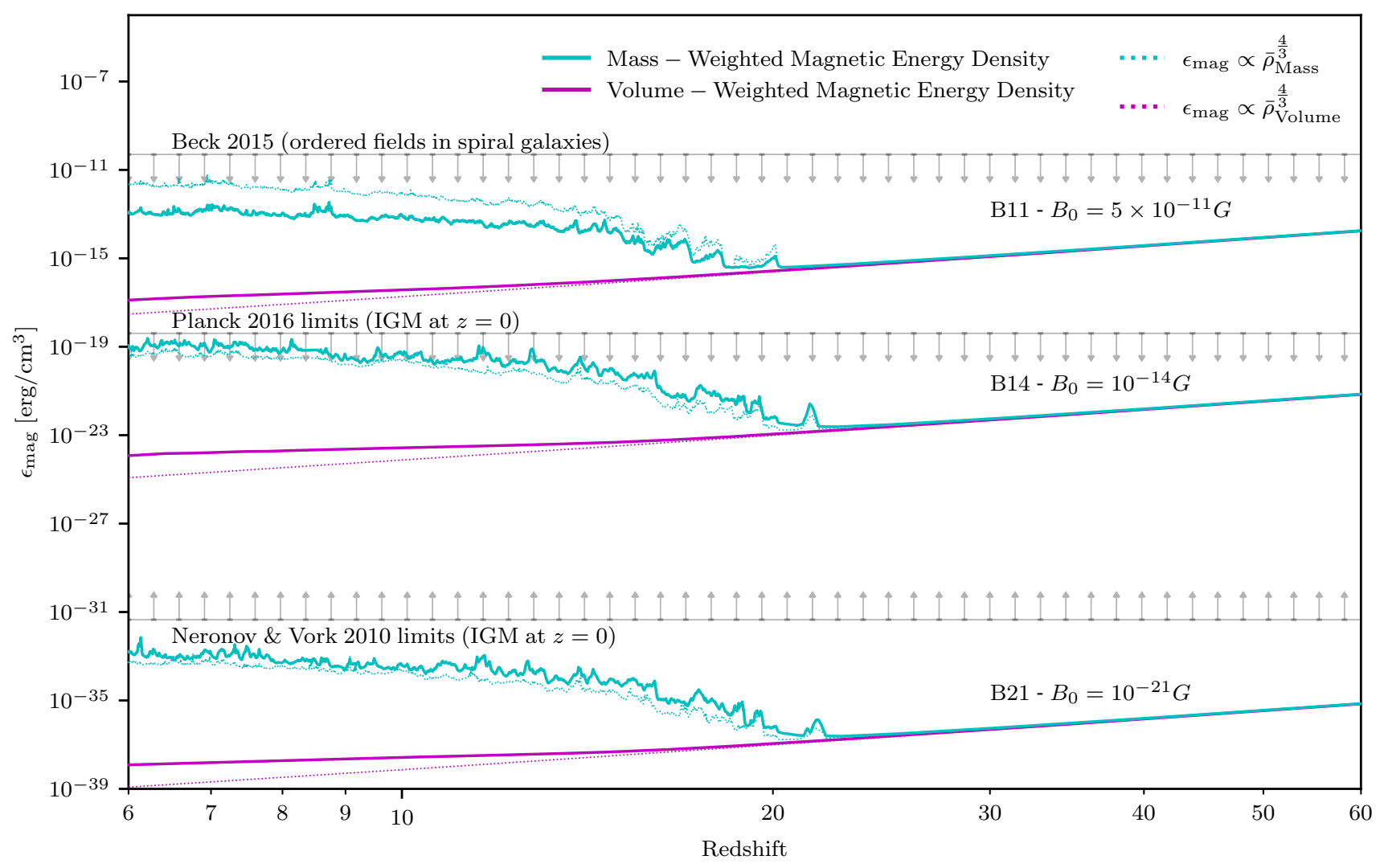

Figure 7. Evolution of the mass-weighted (cyan) and volume-weighted (magenta) energy-density in the magnetic field as a function of redshift for each of the simulations that employ a uniform PMF. The solid lines represent the values calculated directly from the simulation while the dotted lines represents the expectation from flux conservation (i.e. $B \propto \rho^{2 / 3}$ ). The energy density dissipates with time due to cosmological expansion but as soon as the first structures collapse at $z \sim 22$, the mass-weighted magnetic energy density deviates from cosmological expansion. The fact that the solid line is consistently above the dotted line indicates that additional amplification beyond adiabatic compression is occurring in the simulation except for the mass-weighted measurement in B11, indicating that the magnetic energy is saturated in the densest regions of this simulation (i.e. galaxies).

which post-shock gas in a magnetised cloud can collapse against magnetic pressure support (Hennebelle \& Chabrier 2011; Padoan \& Nordlund 2011) so that

$s_{\text {crit }}=\ln \left(0.067 \theta^{-2} \alpha_{\text {vir }} \mathcal{M}^{2} f(\beta)\right)$.

In this equation, $\alpha_{\text {vir }}=2 E_{\text {kin }} /\left|E_{\text {grav }}\right|$ is the virial parameter, $\theta=0.35$ (Hennebelle \& Chabrier 2011), $\mathcal{M}$ is the mach number, and $f(\beta)$ is a dimensionless quantity defined in Padoan \& Nordlund (2011) as

$f(\beta)=\frac{\left(1+0.925 \beta^{-3 / 2}\right)^{2 / 3}}{\left(1+\beta^{-1}\right)^{2}}$.

Thermo-turbulent star formation prescriptions of this ilk have already been described in Kimm et al. (2017); Trebitsch et al. (2017); Rosdahl et al. (2018) and the MHD extension has been used by Katz et al. (2019); Martin-Alvarez et al. (2020b,a). Note that, in contrast to the original SPHINX simulations, we do not include the stellar and dark matter density when calculating the local density to measure the star formation efficiency. Dark matter particles are sparsely sampled in the ISM and the mass of the star particle impacts the local efficiency. Thus for these simulations, we choose to only consider gas density. This leads to reionization occurring slightly later.

\subsection{Stellar Feedback}

Star particles in the simulation can explode via SN throughout the first 50 Myr of their life. We randomly sample a realistic delay-time distribution over this time period to determine when the $\mathrm{SNe}$ occur. We use the mechanical feedback scheme of Kimm et al. (2015) as was also used in SPHINX to inject momentum into the surrounding cells of the star particle depending on resolution. The aim is to inject the final snowplow momentum of a SN remnant if the adiabatic phase is unresolved, or to allow the remnant to evolve naturally if the adiabatic phase is resolved. For each individual SN event, the equivalent of $10^{51} \mathrm{ergs}$ is injected. We adopt a Kroupa (2001) stellar initial mass function (IMF) and recycle $20 \%$ of the total mass of every star particle back into the simulation as gas. Some of this material will be metal enriched due to nucleosynthesis in the stars and we assume that $7.5 \%$ of the ejecta is in the form of elements heavier than hydrogen and helium. For a standard Kroupa (2001) IMF, with a maximum stellar mass of $100 \mathrm{M}_{\odot}$, we would expect $\sim 1 \mathrm{SN}$ event per $100 \mathrm{M}_{\odot}$ in stars (i.e. $10 \mathrm{SN}$ per star particle in our simulation). However, in order to reproduce a realistic UV luminosity function and stellar mass-halo mass relation in the early Universe (Garel et al. 2021), we follow the approach of Rosdahl et al. (2018) and boost the number of SNe per star particle by a factor of four (i.e. $40 \mathrm{SN}$ explosions per star particle) as was done for SPHINX.

In addition to momentum, we inject radiation that emanates from 

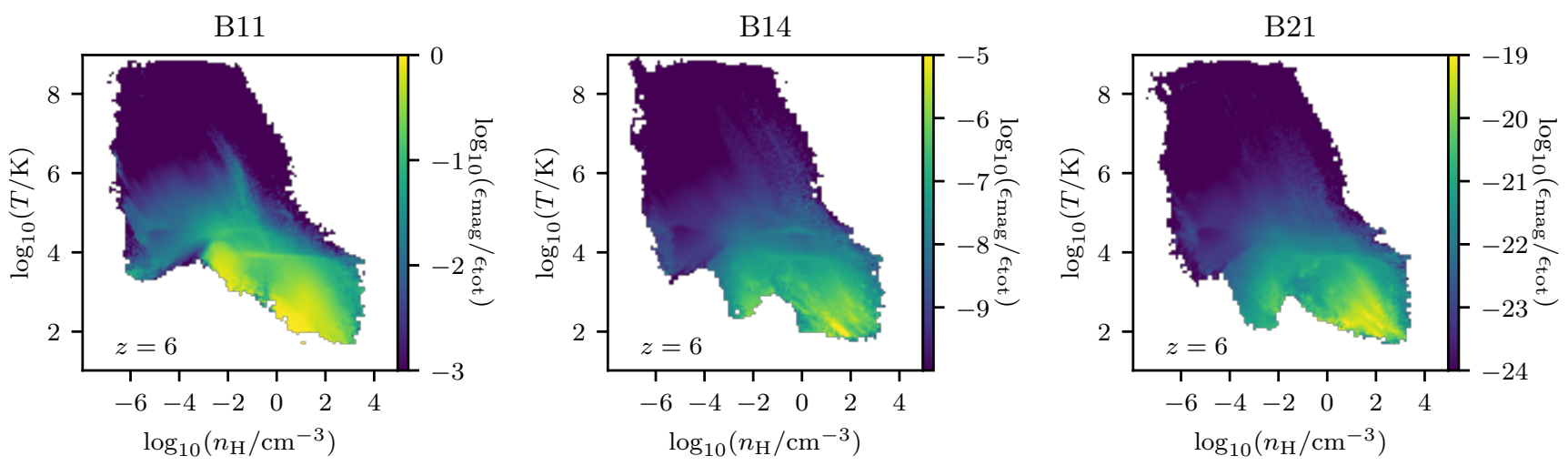

Figure 8. Temperature-density phase space diagrams of the entire simulation volume coloured by the fraction of the total energy in the bin (magnetic, turbulent kinetic, and thermal) that is represented by the magnetic component. For the B14 and B21 simulations, the magnetic energy never reaches equipartition with either the thermal or turbulent kinetic energy across the entire phase-space. In contrast, for the B11 simulation, in certain regions of phase-space, particularly at intermediate densities and temperatures, the magnetic component can become dynamically important and represent $>1 \%$ of the total energy. For individual galaxies, this diagram will appear different.

every star particle in the simulation. The amount of radiation injected on each fine time step in the simulation is calculated using spectral energy distributions computed for binary stellar populations (BPASSv2, Eldridge et al. 2008; Stanway et al. 2016) for a stellar IMF with a maximum mass of $100 \mathrm{M}_{\odot}$. The effects of binary stellar populations are now well studied in the context of reionization (Stanway et al. 2016; Ma et al. 2016; Rosdahl et al. 2018; Ma et al. 2020) and our fiducial simulations would not reionize without assuming binary stellar populations (Rosdahl et al. 2018) unless we modified the subgrid escape fraction (i.e. a parameter representing the fraction of photons that escape the unresolved molecular cloud ${ }^{4}$ ). For this work, we have set the subgrid escape fraction to 1; hence, we do not modify the total number of photons injected into each cell as computed from the age and metallicity dependent SED. For the simulations with PMFs that reionize early due to the enhancement in the number of dwarf galaxies, adopting an SED with fewer ionising photons or an SED with LyC production more biased towards young stellar ages for the stellar population (as is the case without binary stars) may help bring the simulations in better agreement with observations. However the primary goal of this work is to study the impact of magnetic fields on reionization rather than to develop a model that is in perfect agreement with all observational constraints.

\subsection{Halo Finding}

To identify haloes in the simulation, we use the ADAPTAHOP algorithm using the most massive submaxima (MSM) mode (Aubert et al. 2004; Tweed et al. 2009). We identify all haloes and subhaloes that are represented by at least 20 dark matter particles; however, for the analysis in the work, we only consider haloes that contain at least 300 dark matter particles (i.e. $M_{\text {halo }}>6.24 \times 10^{6} h^{-1} \mathrm{M}_{\odot}$ ). The virial mass of each halo is determined by fitting a triaxial ellipsoid to each halo and subhalo with a centre located at the densest region of the halo and iteratively decreasing the volume of the halo until the virial theorem is satisfied. This method produces haloes that have similar virial masses and radii to adopting a spherical overdensity criteria of $200 \rho_{\text {crit }}$ (Rosdahl et al. 2018). We assign star particles

\footnotetext{
4 This value can be $>1$ if the ionised channels through which photons are
} expected to escape are unresolved by our simulations. to haloes based on their location, i.e. whether they reside within the virial radius of a dark matter halo or subhalo. In case of overlap (i.e. a star particle overlapping with more than one halo) it is assigned only to the closest halo, the distance being measured as $d=r / R_{\mathrm{vir}}$, where $r$ is the distance from the halo centre and $R_{\mathrm{vir}}$ is the virial radius of the halo. We also ignore all sub-halos that are fully contained within their parent halo, and instead assign their stellar particles to the parent halo.

\subsection{Escape Fractions}

We measure LyC escape fractions from every halo that contains stars in every simulation snapshot using the publicly available RASCAS code (Michel-Dansac et al. 2020). We post-process the simulation snapshots by casting 500 rays from each star particle in random directions and measuring the optical depth to neutral hydrogen and helium up to the virial radius of the halo. The escape fraction for each star particle is measured as the average of the escape fraction along each of the 500 rays and the global escape fraction for each snapshot is taken as the luminosity weighted average among all star particles. The choice of 500 rays was found to be a robust estimator by Rosdahl et al. (2018); Katz et al. (2020a) as minimal differences were found when sampling up to 100,000 rays per star particle. Furthermore, we note that Trebitsch et al. (2017) found that measuring $f_{\text {esc }}$ using raycasting in post-processing resulted in very similar results to directly measuring the LyC flux present in the simulation that crosses the virial boundary of the halo. Because we use the variable-speed-oflight approximation, measuring the delay time between the moment when photons were emitted and when they cross the virial boundary becomes very difficult due to the adaptive nature of the grid. Hence ray-tracing is a more robust approach in our simulations.

\section{RESULTS}

We now present our results on the impact of magnetic fields on reionization. For each simulation we save snapshots of the volume 


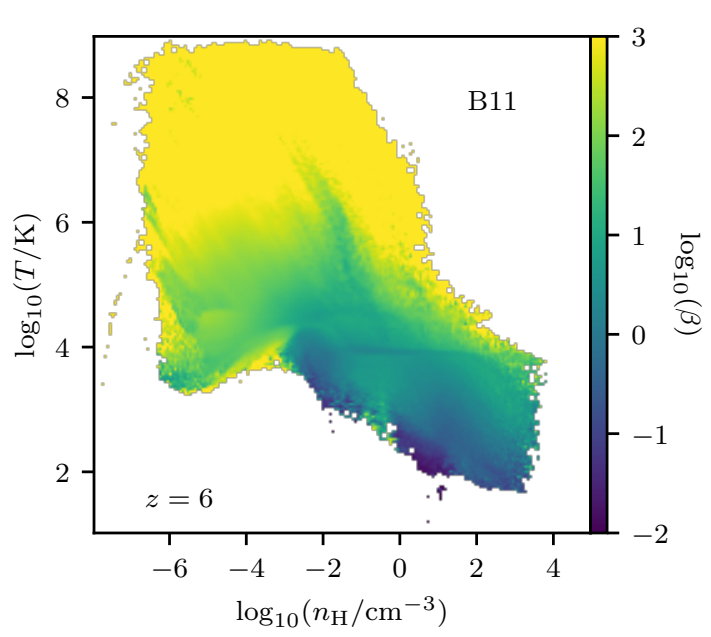

Figure 9. Temperature-density phase space diagram of the B11 simulation at $z=6$ coloured by $\beta$, the ratio of thermal to magnetic pressure. At high densities and low temperatures, $\beta$ drops below one and impacts star formation.

at $5 \mathrm{Myr}$ intervals from the point at which the first stars form ${ }^{5}$ and many of the statistics are computed on-the-fly at every coarse time step in the simulations, providing a time resolution of $\sim 1,000-$ 10,000 years. All simulations have been evolved to $z=6$ except the B11_24 simulation which was evolved until it reached a volumeweighted ionisation fraction of $50 \%$ at $z \sim 55$.

\subsection{Evolution of the magnetic field}

In Figure 6 we show a map of the gas column density and densityweighted magnetic field strength at $z=6$ for the B14 simulation. The gas density exhibits a rich filamentary structure that mimics the dark matter density field seen in Figure 3, except for the least persistent filaments that are the least self-shielded to reionization (Katz et al. 2020b). The large scale magnetic field clearly follows the density field as expected due to flux conservation as the gas collapses onto filaments and is fed into galaxies. Evidence for strong SN feedback is visible around the locations of many galaxies as bipolar outflows and density cavities. For the most massive halo in the simulation that resides in the centre of the image, we can see that the magnetic field is dragged out of the galaxy along with the gas by the SN feedback and into the low-density IGM.

In Figure 7 we show the evolution of the mass-weighted and volume-weighted magnetic energy density in cyan and magenta, respectively, as a function of redshift for the B21, B11, and B14 simulations. In all simulations, the mean strength of the magnetic field decreases with redshift due to cosmic expansion such that $B \propto(1+z)^{2}$. This holds until the collapse of the first galaxy where in Figure 7 , we observe that the mass-weighted magnetic energy density begins to deviate from the volume-weighted magnetic energy density at $z \sim 23$ for the B21 and B14 simulations. First collapse is slightly delayed in the $\mathrm{B} 11$ simulation where the additional pressure support from the magnetic field temporarily prevents runaway collapse of the gas (Martin-Alvarez et al. 2020b). As the Universe evolves and more galaxies collapse, the mass-weighted magnetic energy density

\footnotetext{
5 This results in $\sim 150$ total snapshots for each simulation, corresponding to
} $\sim 40-100 \mathrm{~Tb}$ of data per simulation.

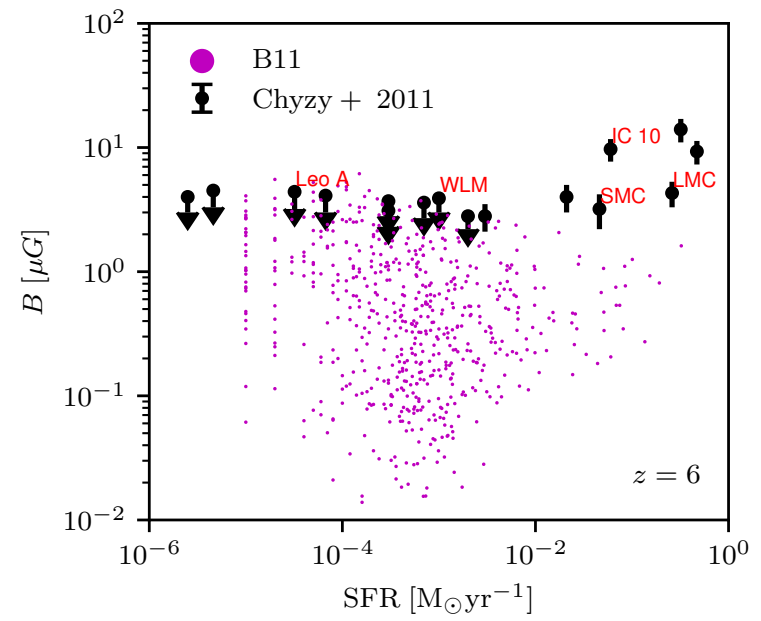

Figure 10. Mass-weighted average magnetic field inside haloes versus 100 Myr-averaged SFR in the B11 simulation at $z=6$ compared to those in local group dwarf galaxies.

continues to increase in all simulations. The dotted lines in Figure 7 represent the expectations from flux conservation (i.e. $\epsilon_{\operatorname{mag}} \propto \rho^{-4 / 3}$, where $\rho$ is either the volume-weighted or mass-weighted average gas density in the simulation). For the B21 and B14 simulations, the mass-weighted magnetic energy density remains consistently higher than the expectation from flux conservation indicating that other mechanisms (e.g., turbulent or rotational motions) act to amplify the magnetic field. We emphasise that numerical viscosity and diffusion as well as limited resolution and an adaptive grid prevent us from reaching the Reynolds numbers needed to sustain significant turbulent amplification. We can estimate the effective Reynolds number as $\operatorname{Re}=(L / \epsilon \Delta x)^{4 / 3}$, with $L$ being the typical length scale or turbulent injection scale of the system and $\epsilon \sim 7$ (Donnert et al. 2018). Setting $L=0.1 r_{\text {vir }}$, we find $\operatorname{Re} \sim 4^{6}$ for galaxies in our simulation assuming that the entire central region of the galaxy is resolved that the maximum spatial resolution. This mean value is below the critical Reynolds numbers of $30-35$ needed to trigger a turbulent dynamo for a magnetic Prandtl ${ }^{7}$ number of $\sim 1$ (e.g. Haugen et al. 2004a,b; Brandenburg \& Subramanian 2005). Hence we do not expect extreme deviations between the measured magnetic energy and that predicted from flux conservation. The most resolved systems have Reynolds numbers above the estimated critical value which perhaps explains the enhancement above flux conservation that we observe in the B14 and B21 simulations.

Significant deviations between the measured and flux conservation predictions for the mass-weighted magnetic energy density are also observed for the B11 simulation where the PMF is strong enough that the magnetic energy becomes saturated inside haloes. In all three simulations, after first collapse, the volume-weighted magnetic energy density increases above the predicted value as the galaxies decouple from the expanding background and magnetic energy is ejected from the galaxies and into the IGM. Observational lower

\footnotetext{
${ }^{6}$ Using an alternative estimate for the Reynolds number, $2 L / \Delta x$ (Rieder \& Teyssier 2017), we find mean values of Re in our simulation of $\sim 40$ (assuming the entire central region of the galaxy is resolved at the highest resolution) which are slightly above the critical value.

7 The ratio between viscosity and magnetic diffusivity.
} 


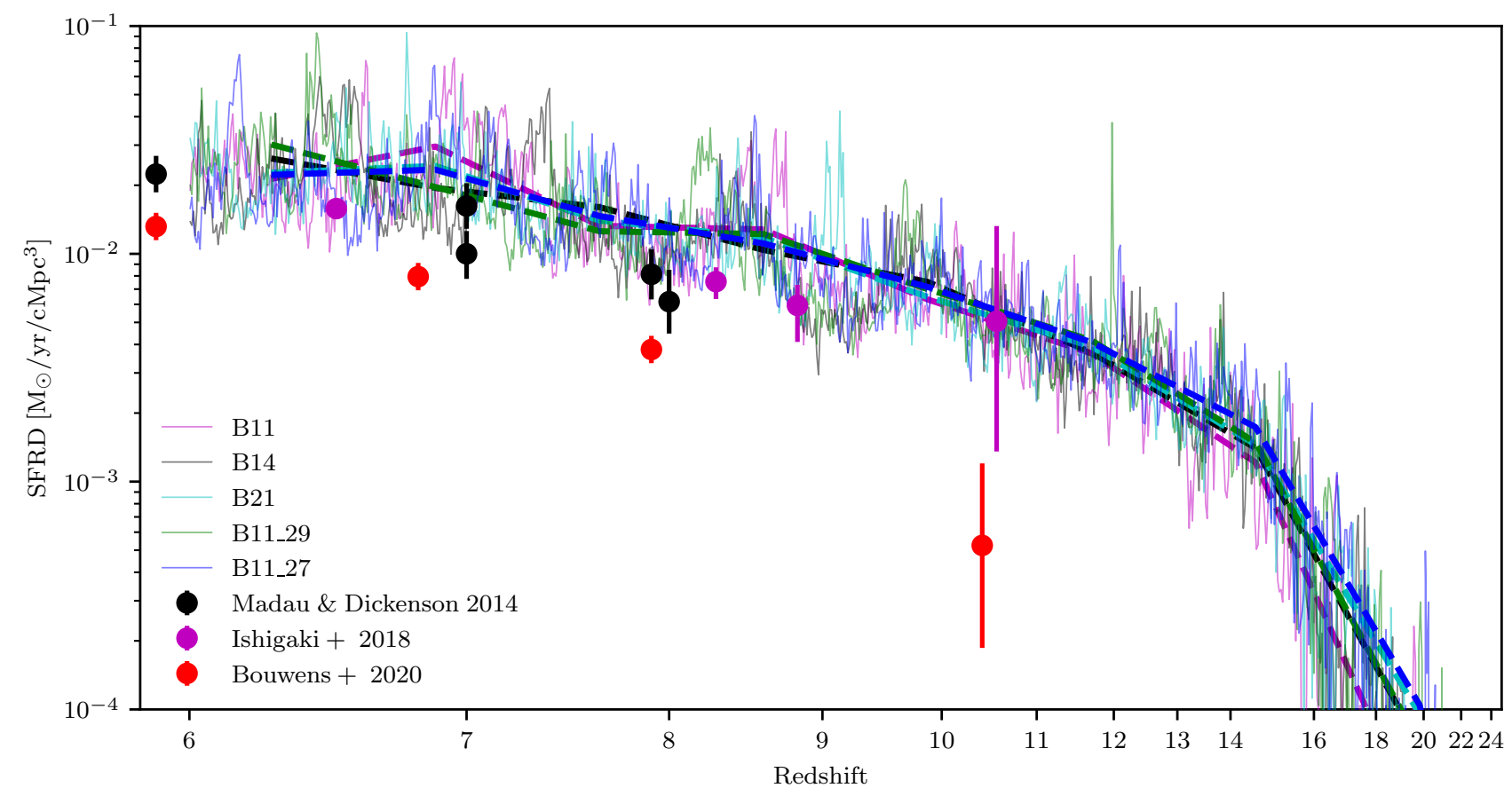

Figure 11. Star formation rate density (SFRD) as a function of redshift for our simulations compared to observational estimates from Madau \& Dickinson (2014); Ishigaki et al. (2018); Bouwens et al. (2020). Solid lines represent the 1Myr-averaged SFRD while dashed lines represent the 100Myr-averaged SFRD.

limits have been estimated using the TeV emission from blazars and their GeV secondary emission (Neronov \& Vovk 2010; Dolag et al. 2011), but their interpretation requires further investigation (Broderick et al. 2012, 2018). For scenarios where the PMF results in an IGM magnetic field weaker than such proposed lower limits (e.g., our B21 simulation), invoking astrophysical phenomena, such as magnetised winds would be required for consistency with such constraints. In contrast, if the filling factor of the IGM magnetic field was shown to be robustly $\ll 1$, this may rule out strong magnetic fields where the volume-weighted magnetic field strength is higher than any upper limit set by observations. In all of our simulations, the volume filling factor of magnetic fields is equal to 1 because of how they were initialised. Scenarios accounting for cosmological perturbations of the magnetic field by e.g. modelling an initial magnetic spectrum, such as our B11_27 and B11_29 runs, might still be found to be in agreement due to the spatial fluctuations depending on the exact volume filling factors and magnetic field strengths observed in the IGM.

Within the ISM of galaxies, weak magnetic fields are expected to be amplified by a turbulent dynamo until their saturation at a fraction of the turbulent kinetic energy. In our simulations, saturation will not occur at equipartition due to the drastic difference between the Prandtl and viscous and magnetic Reynolds numbers achievable in our simulation compared to the real ISM (Martin-Alvarez et al. 2018). Numerical simulations show that saturation occurs when the magnetic pressure reaches $\sim 2 \%-5 \%$ of the turbulent energy for Prandtl numbers of $O(1)$ (e.g., Federrath et al. 2014; Tricco et al. 2016; Rieder \& Teyssier 2017), although saturation fractions may exhibit a much wider range depending on Prandtl and Mach numbers (e.g. Schober et al. 2015; Chirakkara et al. 2021). In Figure 8 we show temperature-density phase-space diagrams of all the gas in the box at $z=6$ for the B21, B14, and B11 simulations, coloured by the ratio of magnetic energy to total energy (thermal, turbulent kinetic, and magnetic). We use a simple approximation for the turbulent kinetic energy and define it as $\frac{1}{2} m_{\text {gas }} \sigma^{2}$, where $m_{\text {gas }}$ is the gas mass of the cell and $\sigma$ is the velocity dispersion. For the B14 and B21 simulations, the magnetic energy remains far below both the thermal and kinetic energy indicating that it is not dynamically important in any region of the phase space. Interestingly, we can see some striations in the high-temperature and low-density regions of the panels for these simulations. These features represent individual SN events dragging the magnetic field out of galaxies. In contrast, in the B11 simulation, we can identify localised regions of the temperature-density phase space where the magnetic field is dynamically important and represents more than $50 \%$ the total energy. In particular, this occurs often at $T \lesssim 10^{4} \mathrm{~K}$, inside of galaxies.

Perhaps more interesting in the context of star formation is the value of $\beta$ (i.e. the ratio of thermal to magnetic pressure) in lowtemperature, high-density star-forming gas. In Figure 9, we plot a $2 \mathrm{D}$ histogram of density and temperature coloured by the values of $\beta$. In star forming regions, $\beta$ drops to values $\ll 1$, consistent with observations of local molecular clouds where typical values can range from $\beta \sim 10^{-3}-10^{-1}$ (Crutcher 2012; Krumholz \& Federrath 2019). Clearly, the magnetic field in the B11 can have a drastic impact on our $\mathrm{SF}$ recipe. In contrast, the B14 and B21 simulations exhibit values of $\beta \gg 1$ and hence for these simulations, the additional magnetic pressure does not impact star formation.

In Figure 10, we compare the mass-weighted magnetic field strengths inside of haloes in the B11 simulation at $z=6$ with estimates for the magnetic field strengths of local group dwarf galaxies as a function of SFR (Chyży et al. 2011). Interestingly, for the galaxies with low SFR (i.e. SFR $<10^{-2} \mathrm{M}_{\odot} \mathrm{yr}^{-1}$ ), our measurements appear 

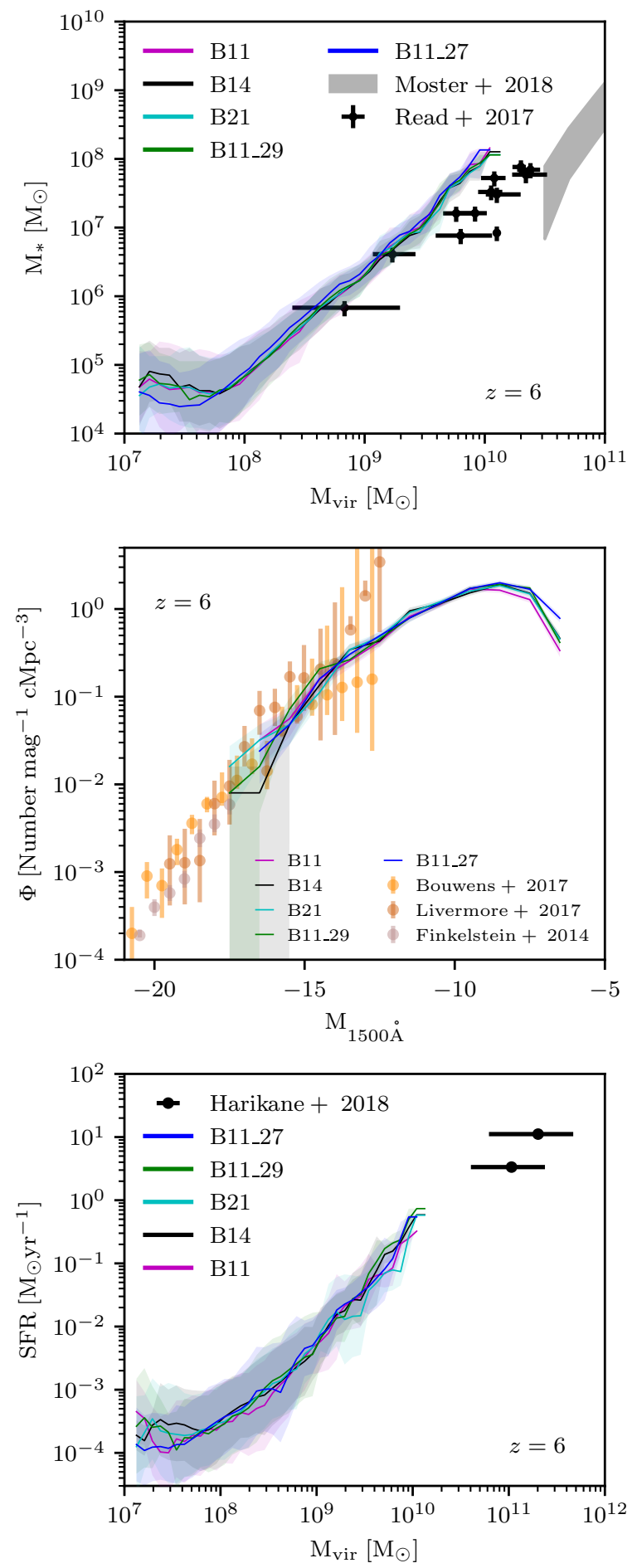

Figure 12. Stellar mass-halo mass relation compared to local group dwarf galaxies (Read et al. 2017) and constraints from abundance matching (Moster et al. 2018) (top), 1500£ UV luminosity function compared to observational constraints from Finkelstein et al. (2015); Livermore et al. (2017); Bouwens et al. (2017b) (centre), and SFR-halo mass relation compared to observational constraints from Harikane et al. (2018) (bottom), for each of the simulations at $z=6$. In the top and bottom panels, lines and shaded regions represent the running means and $1 \sigma$ scatter around the relation. consistent with the constraints from local dwarfs. In contrast, the galaxies in our simulation with higher SFRs tend to exhibit magnetic field strengths over the whole halo that are lower than local galaxies such as the LMC and SMC. A detailed comparison between observed and simulated magnetic fields would require studying in better detail the properties of the gas inside galaxies and its synchrotron emission, which is beyond the scope of this work. However, the limited resolution of our simulations might be inhibiting both the growth rate and maximal saturation strength of magnetic fields in the simulated galaxies (Rieder \& Teyssier 2017; Martin-Alvarez et al. 2018). Furthermore, there is no reason a priori for dwarf galaxies in the epoch of reionization to exhibit magnetic field strengths similar to those in Local Group dwarf galaxies. ISM conditions are expected to be significantly different as high-redshift galaxies are likely more compact, gas rich, and turbulent, while $z=0$ galaxies have had significantly more time to evolve, amplify, and organise on galactic scales their seed magnetic fields. Even for weak seed fields (i.e. $B \sim 10^{-20} G$ ), saturation at levels of $\gtrsim 10^{-6} G$ are predicted within the free-fall time of a primordial halo (Schober et al. 2012) which is consistent with the saturation levels achieved in our simulations with the strongest PMFs.

Observational constraints on the magnetic fields within galaxies at high redshift remain scant; however, for massive galaxies at $z \sim 3$ where such observations can be made, it seems that these galaxies exhibit magnetic field strengths consistent with those observed at $z=0$ (Bernet et al. 2008), supporting the possibility of magnetic saturation in galaxies at extremely early times in the evolution of the Universe.

Thus far our analysis has focused only on the simulations that are initialised with uniform magnetic fields. The magnetic field evolution in the simulations with randomly seeded fields and specific spectral slopes are not fundamentally different from those already discussed.

\subsection{Impact of magnetic fields on star formation}

One of the primary goals of this work is to better understand how the presence of PMFs impacts galaxy formation in the epoch of reionization. In this Section, we focus on how PMFs impact star formation.

In Figure 11, we show the star formation rate density (SFRD) for each of the simulations compared with the observational estimates from Madau \& Dickinson (2014); Ishigaki et al. (2018); Bouwens et al. (2020). In all simulations, the onset of star formation occurs at $z \sim 20$ and increases rapidly until $z \sim 12-14$. As more haloes form stars, stellar feedback regulates the formation of new stars and the SFRD settles to a value slightly higher than $\sim 10^{-2} \mathrm{M}_{\odot} / \mathrm{yr} / \mathrm{cMpc}^{3}$ by $z=6$, which is consistent with observations. Due to the small volume of the simulation, the SFRD continues to fluctuate by factors up to an order of magnitude, even at $z<7$ as intense star formation events in the massive galaxies can dominate the star formation rate of the simulation volume. It should be noted that the observational estimates of the SFRD from Madau \& Dickinson (2014); Bouwens et al. (2020) are calculated up to a limiting magnitude which is barely probed by our simulation as a consequence of the small volume. Hence the observational data point at $z=10.5$ falls far below our simulated data, where lower luminosity galaxies are expected to contribute significantly to the star formation rate (e.g. Behroozi et al. 2020). In contrast, the data from Ishigaki et al. (2018) are integrated to a limiting magnitude of -11 and is much more consistent with our simulations. It is perhaps a coincidence that the brighter galaxies at redshifts closer to 6 probed by observations have a similar SFRD compared to the low mass galaxies in our computational volume. 


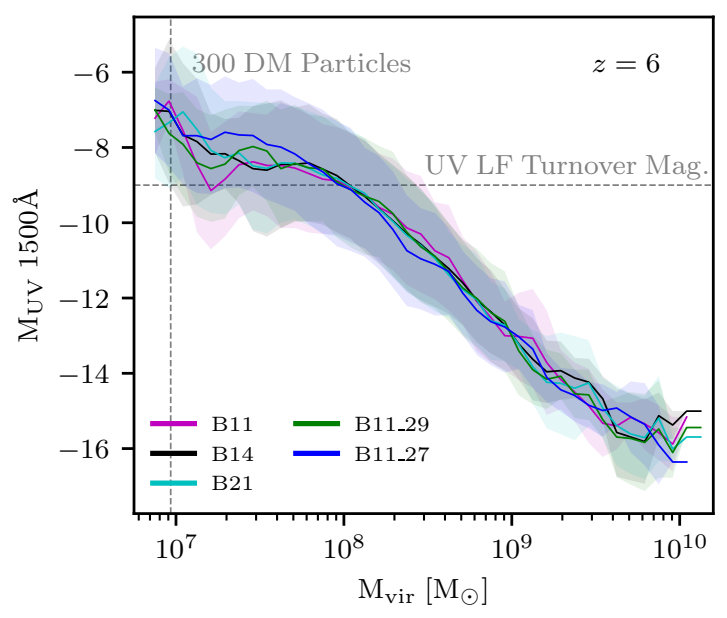

Figure 13. Virial mass versus $1500 \AA$ UV luminosity for each of the simulations at $z=6$. The lines and shaded regions represent the running means and $1 \sigma$ scatter around the relation.

For the strengths of the PMF sampled in this work, we do not see a significant impact on the total amount of star formation in the simulations. The evolution of the SFRD is consistent across simulations, regardless of the strength of the PMF. Similarly, the total stellar masses formed in the simulations are all within a factor of two by $z=6$. This behaviour is consistent with our earlier work Katz et al. (2019); Martin-Alvarez et al. (2020b) as well as others that have demonstrated that the stellar content of galaxies is not impacted for PMFs with $\lesssim 10^{-9} G$ (e.g. Marinacci \& Vogelsberger 2016) nor does seeding mechanism play a substantial role (Garaldi et al. 2020).

In the top panel of Figure 12 we show the stellar mass-halo mass relation at $z=6$ for each simulation compared to $z=6$ estimates from abundance matching (Moster et al. 2018) as well as individual local group dwarf galaxies (Read et al. 2017). The lines and shaded regions represent the running mean of stellar mass and the $1 \sigma$ standard deviation for haloes that host at least one star particle. Note that the fraction of haloes that host a stellar population at a halo mass of $10^{8} \mathrm{M}_{\odot}$ is only $50 \%$. In general, the simulations are in very good agreement with each other and exhibit a slightly steeper relation compared with local dwarf galaxies. Below halo masses of $10^{8} \mathrm{M}_{\odot}$ there is a small deviation between the B11_27 simulation and the other runs such that, on average, the stellar mass for a given halo mass is lower in this run.

This is more easily observed in the second panel of Figure 12 where we show the $1500 \AA$ luminosity function for each of the simulations at $z=6$ compared to observations from Finkelstein et al. (2015); Livermore et al. (2017); Bouwens et al. (2017b). At UV magnitudes brighter than -13 , where the simulations overlap with observations, we find very good agreement between our predictions and observations. There is some disagreement in the observational data at magnitudes fainter than -15 where observational estimates are derived from lensed galaxies where magnification uncertainties can be substantial and observational volumes are small. Nevertheless, our predictions seem to fall in between the different observational constraints. At the faintest magnitudes (i.e. $>-9$ ), the luminosity function turns over. We caution that this is partially due to limited resolution in the simulation, both because the haloes are resolved by fewer than 10,000 DM particles, and the minimum stellar mass in the simulations is $1000 \mathrm{M}_{\odot}$. We demonstrate this further in Figure 13 where we plot the relation between virial mass an $1500 \AA \mathrm{UV}$ magnitude. The turnover in the LF occurs at a UV magnitude of -9 which corresponds to a virial mass of $\sim 10^{8} \mathrm{M}_{\odot}$. Such haloes are resolved by $\sim$ 3, $000 \mathrm{DM}$ particles which is sufficient to resolve some of their DM and gas properties but only marginally resolve the stellar content (e.g. Brooks et al. 2007). The feedback from the process of reionization has the capacity to induce this turn-over; however, the simulations may not have not reached the point where we expect this to occur since the galaxies can self-shield long after reionization (Katz et al. 2020a).

At faint magnitudes, the B11_27 simulation exhibits a slightly higher number density of galaxies, consistent with the enhancement in the number of low mass dark matter haloes. The differences at the faint-end are larger than the $1 \sigma$ uncertainty. Furthermore, comparing the B11_27 and B14 simulations UV magnitude distributions with a two-sided KS tests results in a $p$-value of 0.0038 indicating that they were likely drawn from a different underlying distribution. The enhancement in faint galaxies combined with the tendency of strong magnetic fields to delay star formation in haloes translates to a small decrease in the average stellar mass at halo masses $<10^{8} \mathrm{M}_{\odot}$ in the top panel of Figure 12. Similar behaviour was observed in the zoomin simulations of Sanati et al. (2020) at $z=0$ where data from the Local Group was used to constrain both the strength and slope of the PMF.

Note that the UV luminosity functions presented here represent the intrinsic UV magnitudes of the galaxies and do not include the impact from dust. While this is likely a good approximation at such faint magnitudes (Ma et al. 2016), we cannot rule out the possibility that the intrinsically brighter galaxies, which do not exist in our small volume, would be reddened to the brightest magnitude bins represented in our simulations, and hence increase the simulated luminosity function upwards and out of the range of observational constraints. This effect is expected to be mild (Garel et al. 2021).

In the bottom panel of Figure 12 we show the $z=6$ relation between halo mass and the $100 \mathrm{Myr}$-averaged SFR compared with observations from Harikane et al. (2018). Once again, we observe that all simulations exhibit similar behaviour, regardless of the strength of slope of the PMF. This holds true even for the lowest mass haloes with $\mathrm{M}_{\mathrm{vir}}<10^{8} \mathrm{M}_{\odot}$. Unfortunately, the halo masses where observational constraints exist for the $\mathrm{M}_{\mathrm{vir}}-\mathrm{SFR}$ relation are an order of magnitude larger than those probed by our simulation. Nevertheless, consistent with our earlier work (Rosdahl et al. 2018), extrapolating the trends seen in the simulations presented here would slightly overpredict the few constraints that we have from observations. However, including dust obscuration may bring the simulations into agreement with observations (Garel et al. Submitted).

\subsection{Magnetic Fields and Galaxy Structure}

The galaxy size-luminosity relation is one of the primary uncertainties in determining the completeness of galaxy surveys at high redshift. This in turn impacts our estimates of the UV luminosity function, especially at the faint-end where current surveys are expected to be incomplete (e.g. Bouwens et al. 2017a). Hence, constraining this relation is important, both for current and upcoming surveys.

Martin-Alvarez et al. (2020b) demonstrated that strong PMFs can shrink galaxies by nearly a factor of two as well as reduce the spin parameter of the galaxy. Numerous physical mechanisms can drain angular momentum from the gas and deposit it further away from the centre of the galaxy such as Maxwell stresses (Sparke 1982) or deceleration of inflows (Birnboim 2009). This drives gas closer to the centre of the galaxy; hence reducing its size. In the top panel 

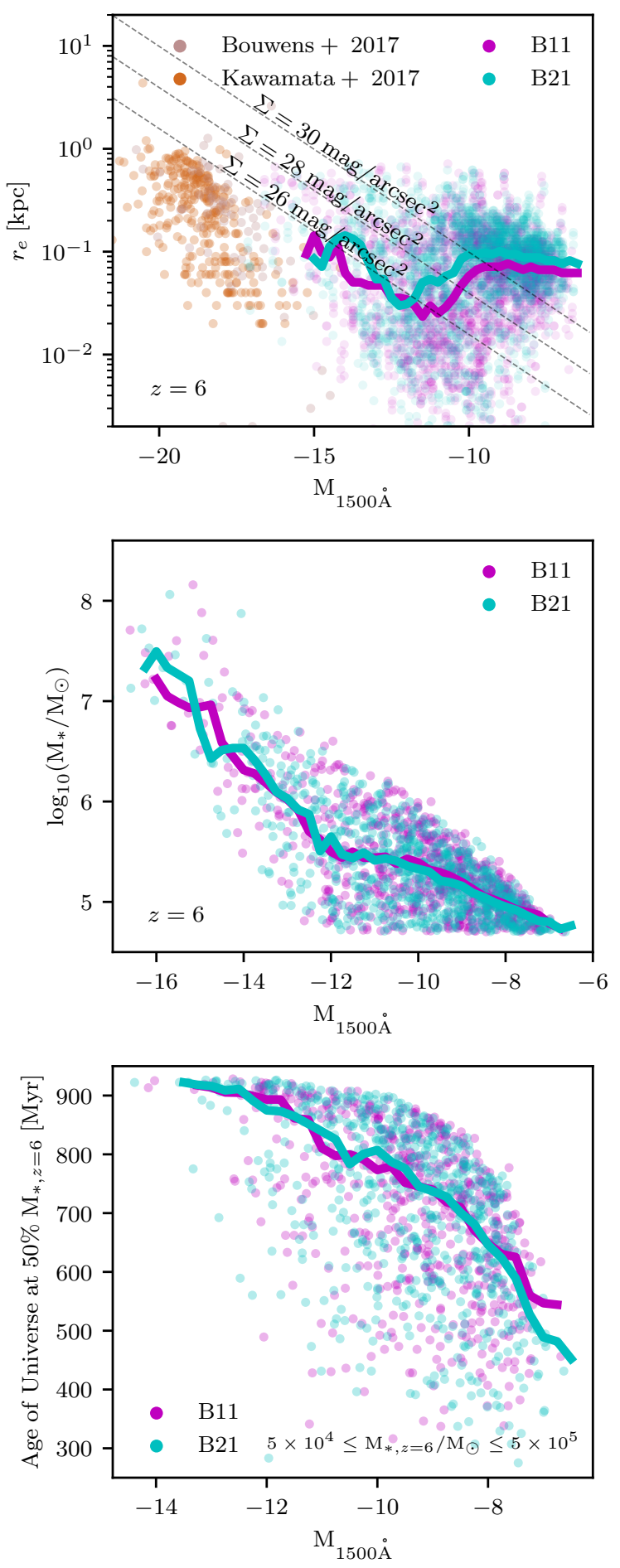

Figure 14. (top) Size-luminosity relation (1500Å UV magnitude versus effective radius) at $z=6$ for the $\mathrm{B} 11$ (strongest magnetic field) and $\mathrm{B} 21$ (weakest magnetic field) simulations compared with the observations from Kawamata et al. (2018); Bouwens et al. (2017a). We calculate the effective radius $r_{e}$ as the $2 \mathrm{D}$ radius that encapsulates half of the light for three different viewing angles of each galaxy that contains at least 50 star particles. The solid lines represent the running medians for each simulation and a bin is only included if it contains at least 30 galaxies. The effective radii in the B11 simulation are generally smaller than those in the B21 simulation indicating that strong galactic magnetic fields can shrink galaxies. Dashed black lines represent constant surface brightness as indicated. (centre) $1500 \AA$ UV magnitude versus $z=6$ stellar mass. (bottom) $1500 \AA$ UV magnitude versus the age of the Universe at which the galaxy formed $50 \%$ of its stars for galaxies with $5 \times 10^{4} \leq \mathrm{M}_{*, z=6} / \mathrm{M}_{\odot} \leq 5 \times 10^{5}$.
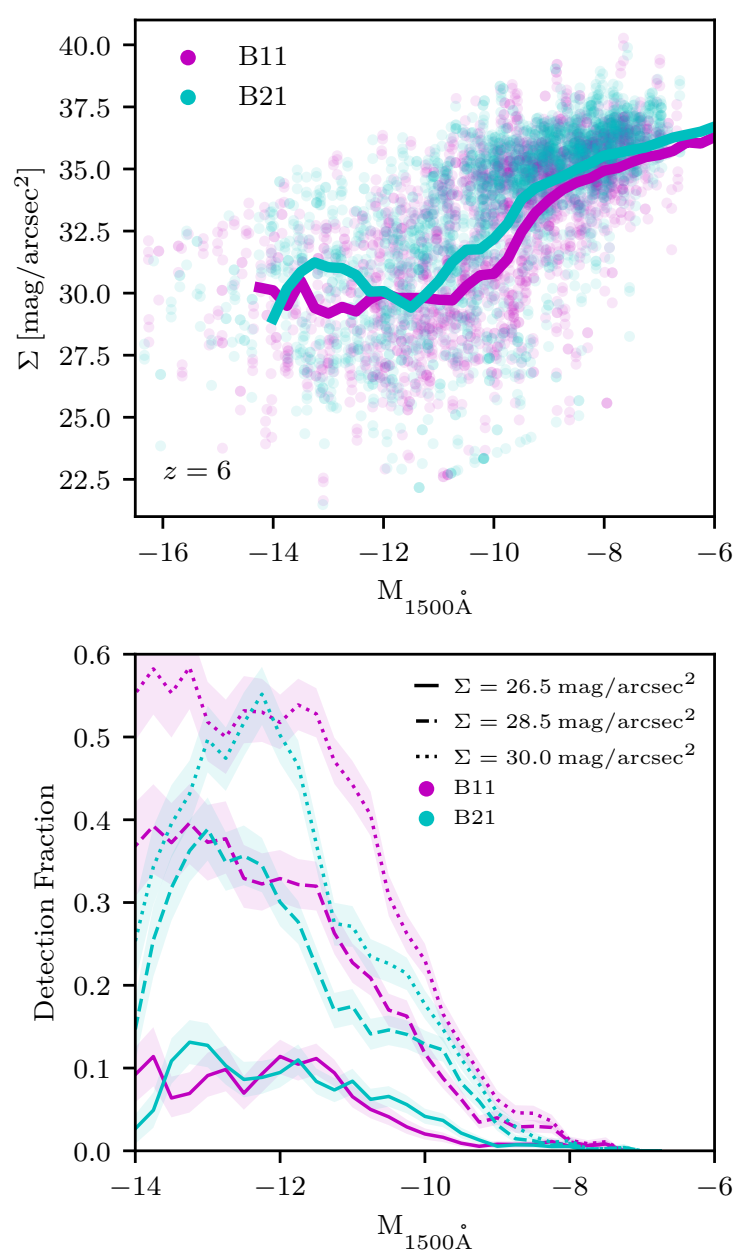

Figure 15. (top) $1500 \AA$ UV magnitude versus apparent surface brightness at $z=6$ for galaxies in the B11 and B21 simulations. (bottom) $1500 \AA \mathrm{UV}$ magnitude versus the fraction of galaxies that could be detected in a surfacebrightness limited survey with limits of 26.5 (solid), 28.5 (dashed), and 30.0 (dotted) mag/arcsec ${ }^{2}$. This does not account for the aperture and magnitude limit of a telescope and only considers surface brightness. The shaded regions represent the $1 \sigma$ standard deviation around the mean for 1,000 bootstrap resamples from the data-set. Because stronger magnetic fields tend to shrink galaxies, they have higher average surface-brightness and are more easily detectable.

of Figure 14 we plot the $1500 \AA$ UV magnitude versus the effective radius $^{8}$ for galaxies in the $\mathrm{B} 11$ (strongest magnetic field) and B21 (weakest magnetic field) simulations at $z=6$ compared with observations from Bouwens et al. (2017a) and Kawamata et al. (2018). The observations are surface brightness-limited, as shown by the dashed black lines, and thus the observed relation appears to be steep. There is good agreement between our simulations and observations in the regime where the data overlap; however, most of the simulated galaxies exhibit UV magnitudes that are far dimmer than what is currently observed.

8 Effective radius, $r_{e}$, is measured by choosing a projection angle for the galaxy centred on the centre of light and finding the radius that encloses half the total light of the galaxy. For each galaxy, we choose three different projection angles along the principle axes of the simulation. Note that we do not include the impact of dust on $r_{e}$ which could impact our results, especially if dust obscuration varies with radius. 
In general, the median galaxy in the simulation with weak PMFs (B21) has an effective radius that is $44 \%$ larger than galaxies of similar absolute magnitude in the simulation with a strong PMF (B11). The scatter is indeed significant due to differences in star formation history, feedback, tidal effects, and halo accretion history and the mean difference between the effective radii, although systematic, are well within the scatter. The large scatter is consistent with expectations from similar reionization-era simulations (Ma et al. 2018). Furthermore, the trend between magnetic field strength and galaxy size expected from the zoom simulations of Martin-Alvarez et al. (2020b) is consistent with what we find in this work. Since surface brightness is proportional to the square of the galaxy radius, the effective decrease in surface brightness for galaxies in the B21 simulation is about a factor of two compared to those in the B11 simulation. This implies that the true completeness fraction of galaxy surveys at faint magnitudes is smaller for a Universe with weak PMFs compared to strong PMFs.

Such an effect is more easily visualised in Figure 15 where in the top panel we show the mean surface brightness of the galaxies within the effective radius $r_{e}$ in the B11 and B21 simulations at $z=6$ for three different viewing angles. Once again we can see the trend that the median galaxy in the B11 simulation has a higher average surface brightness by $\sim 0.7$ magnitudes/arcsec ${ }^{2}$ since they tend to be more compact. As indicated earlier, these differences are still well within the scatter of the relation. In the bottom panel of Figure 15 we show the fraction of galaxies that would be detected for a given surface-brightness threshold. For surface brightness limits of 26.5 mag/arcsec ${ }^{2}$ (approximately equivalent to that of the Hubble Frontier Fields assuming a $5 \sigma$ detection threshold and a 0.4 " diameter aperture Lotz et al. 2017), fewer than $10 \%$ of galaxies could be detected. Such few galaxies are visible at this surface brightness limit that no difference is seen between the B11 and B21 simulations. However, for a surface-brightness limit of $30 \mathrm{mag} / \mathrm{arcsec}^{2}$, considerably more galaxies could be detected at magnitudes fainter than -12 in a Universe with strong PMFs. The deviations seen at higher magnitudes should be interpreted with caution due to the limited number of galaxies in these magnitude bins. Hence stochastic effects from star formation play a role in the detection fraction. Note that real surveys are also magnitude limited so realising this effect will be difficult, even with JWST.

Although galaxies tend to be larger in simulations with weaker PMFs, the general trends between effective radius and UV magnitude hold between the simulations. Effective radius tends to decrease towards fainter magnitudes until $\mathrm{M}_{\mathrm{UV}} \sim-12$, where the effective radius slightly increases and then remains flat. The negative slope observed at the brighter magnitudes is due to the fact that the galaxies are more massive at brighter magnitudes and thus angular momentum conservation makes them larger (e.g. Mo et al. 1998). In the central panel of Figure 14, we plot $\mathrm{M}_{\mathrm{UV}}$ versus galaxy stellar mass at $z=6$ and it is clear that for magnitudes brighter than -12 , stellar mass increases towards brighter UV luminosities. In contrast, at magnitudes fainter than -12 , the slope becomes much more shallow.

We argue that the increase in effective radius towards fainter magnitudes above $\mathrm{M}_{\mathrm{UV}} \sim-12$ is due to galaxies of approximately similar stellar masses ageing towards fainter magnitudes, and expanding due to feedback reducing the gravitational potential. In the bottom panel of Figure 14 we plot the UV magnitude against the age of the Universe at which the galaxy formed $50 \%$ of its $z=6$ stellar population. We only show galaxies that have a stellar mass within the range $5 \times 10^{4} \mathrm{M}_{\odot}-5 \times 10^{5} \mathrm{M}_{\odot}$, which approximately selects for the galaxies with magnitudes fainter than -12 . The upper envelope on this plot results from the magnitude of a fixed stellar population ageing over
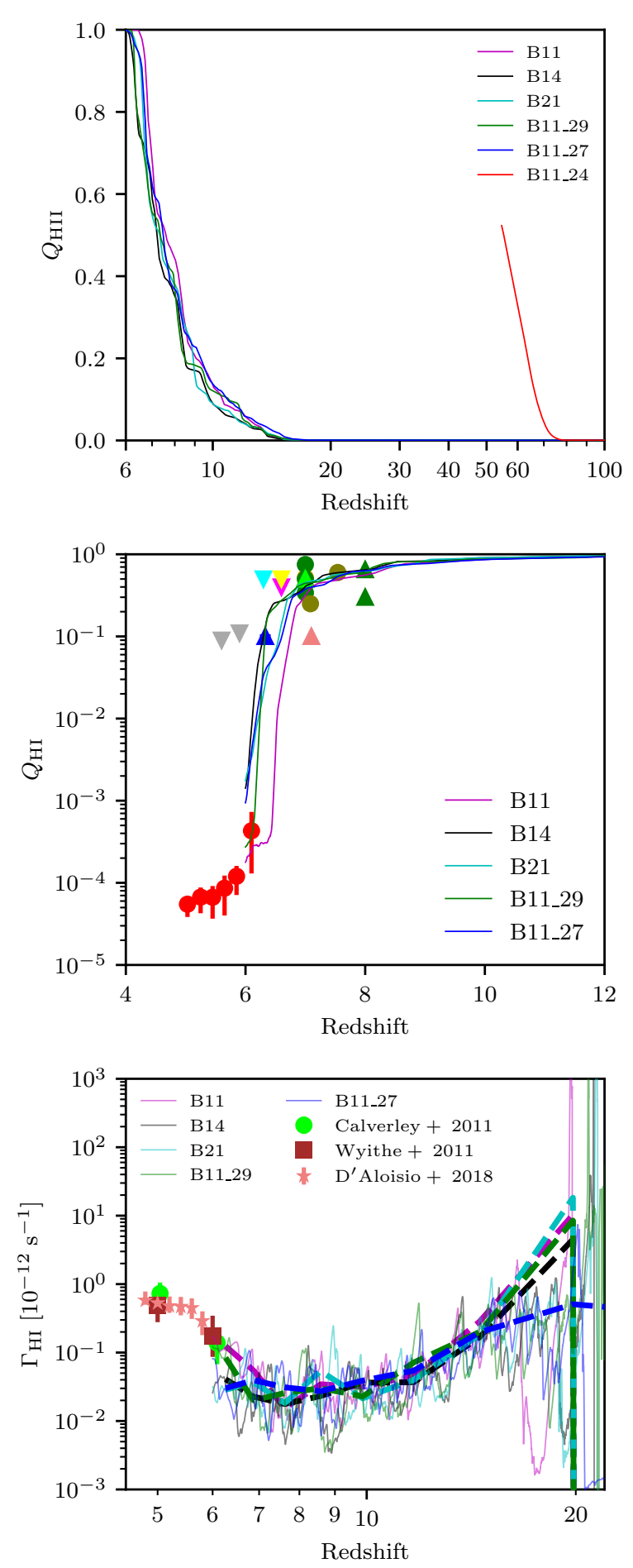

Figure 16. Evolution of the volume-weighted ionised fraction (top), volumeweighted neutral fraction (centre), and the volume-weighted HI photoionization rate (bottom) as a function of redshift for each of the simulations. The dashed lines in the bottom panel represent the 100Myr-averaged values of $\Gamma_{\mathrm{HI}}$. Neutral fraction data points are from the observations and modelling of Fan et al. (2006); McGreer et al. (2015); Schroeder et al. (2013); Schenker et al. (2014); Caruana et al. (2014); Ono et al. (2012); Pentericci et al. (2014); Robertson et al. (2013); Tilvi et al. (2014); Totani et al. (2006); McQuinn et al. (2008, 2007); Ouchi et al. (2010); Ota et al. (2008); Sobacchi \& Mesinger (2015); Mortlock et al. (2011); Bolton et al. (2011); Durovčíková et al. (2020). Photoionization rate data points and models have been compiled from Calverley et al. (2011); Wyithe \& Bolton (2011); D’Aloisio et al. (2018). 


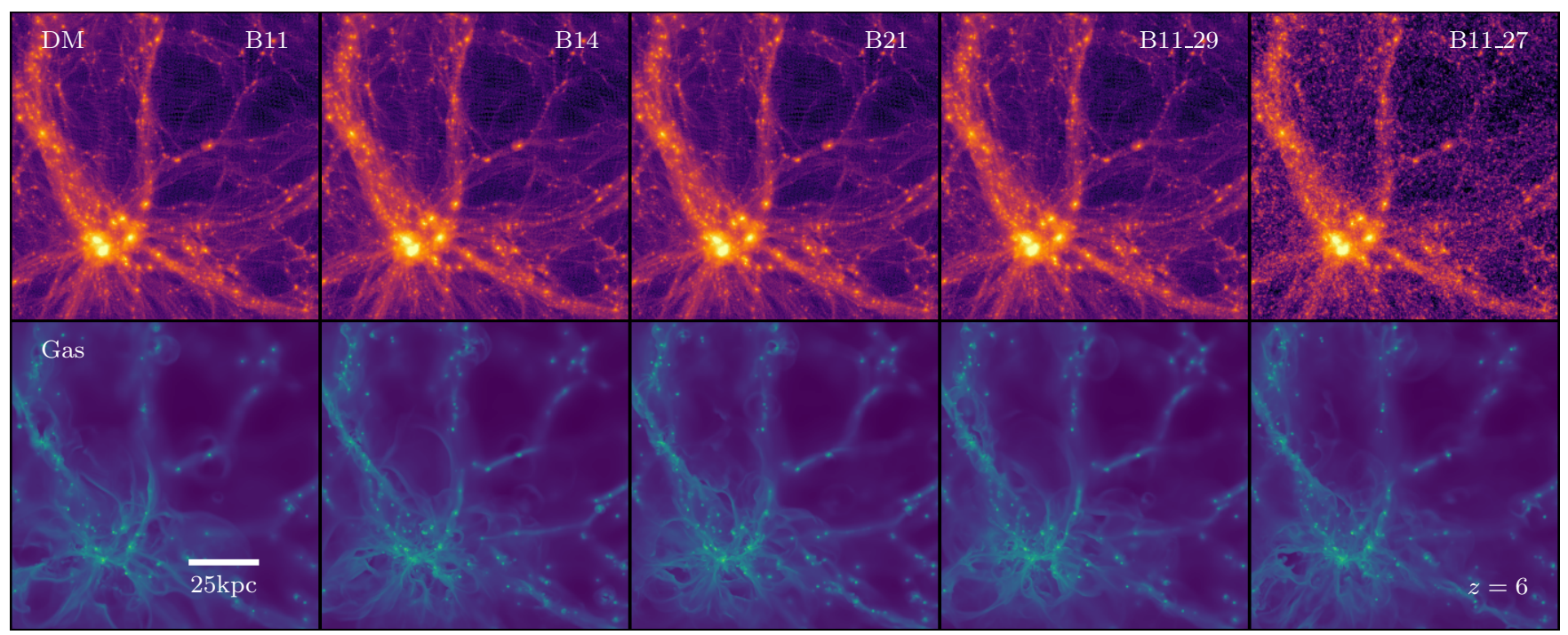

Figure 17. Surface density maps of dark matter (top) and gas (bottom) for the central $25 \%$ of the computation volume at $z=6$ for each of the simulations. The enhancement in number density of low mass dark matter haloes can be seen in the B11_29 and B11_27 simulations; however, such differences are not observed in the baryon distribution.

time while the scatter is due to variations in star formation history. It is clear that the galaxies that formed their stars earlier are much fainter. These galaxies that formed earlier will also be initially more compact; however, stellar feedback can quickly overwhelm the gravitational potential of these galaxies and cause expansion (e.g. Dekel \& Silk 1986; Pontzen \& Governato 2012), consistent with the increase in effective radius seen in the top panel of Figure 14. It is clear from Figure 14 that our simulations do not predict a monotonic trend between size and luminosity. Thus the relations in the literature (e.g. Shibuya et al. 2015) derived from brighter galaxies are not predicted to hold at faint luminosities.

\subsection{Evolution of the intergalactic medium}

Because PMFs can impact both the ISM of galaxies as well as the dark matter halo mass function, we expect that the history of reionization and the properties of the intergalactic medium can change depending on the characteristics of the PMF. Pandey et al. (2015) and Sanati et al. (2020) have already demonstrated that the history of reionization can be used as a constraint on the properties of the PMFs using analytical models and zoom-in simulations, respectively. Furthermore, for strong enough PMFs, we expect to be able to observe their signatures in the properties of the Ly $\alpha$ forest (Chongchitnan \& Meiksin 2014). Our simulations are among the first to study these effects in cosmological radiation magnetohydrodynamics simulations and in this section, we explore the impact of various PMFs on the history of reionization and the properties of the IGM.

In the top panel of Figure 16, we show the ionised fraction $\left(Q_{\mathrm{HII}}\right)$ as a function of redshift from $z=100$ to $z=6$ for all of the simulations. Most notably, the B11_24 simulation is already substantially reionized at $z>50$, inconsistent with nearly every observational probe of reionization. The strong enhancement of the power spectrum at high $k$-modes in the B11_24 simulation leads to the early collapse of a substantial number of dwarf galaxies and thus a very early reionization history. Due to computational limitations, we have only evolved this simulation until $Q_{\mathrm{HII}}=50 \%$. Our results demonstrate that we can rule out PMFs with $B_{0}=5 \times 10^{-11} G$ and spectral indices $n_{B}>-2.4$. Such findings are qualitatively consistent with Sanati et al. (2020) who used standard hydrodynamics simulations with a modified initial density field and also found that initial conditions produced with magnetic fields with $B_{0}=5 \times 10^{-11} G$ and $n_{B}>-2.4$ resulted in reionization histories that are inconsistent with observations. However, our simulations seem to reionize considerably earlier than their semi-analytic approach based on the SFHs from their cosmological hydrodynamic simulations. We provide more detailed constraints on the properties of the PMF in Section 3.6.

In contrast to the B11_24 simulation, all other models exhibit a reasonable reionization histories that complete at $z \sim 6$. In the centre panel of Figure 16 we plot the neutral fraction for each of the simulations as a function of redshift where we see that nearly all simulations reach $Q_{\mathrm{HI}} \lesssim 10^{-3}$ by $z=6$. This is consistent with the majority of models and observations in the literature, although this is perhaps slightly early compared to more recent models (Kulkarni et al. 2019; Keating et al. 2020) that seem to point towards a later reionization, completing at $z \lesssim 5.5$.

As was discussed earlier, there are few differences between the simulations in terms of their stellar content. Hence it is not surprising that the reionization histories are consistent between the simulations. The B11 simulation, which has the highest amount of energy stored in the magnetic field, reionizes very slightly early compared to the other models, possibly due to differences in ISM structure of the galaxies as shown in Section 3.3. We note that, due to the small volume of the simulation, re-running the simulation with a different random seed for the star formation subroutine would also result in small changes in the reionization history due to differences in the star formation history. More interestingly, despite the significant increase in the number density of dwarf galaxies with $\mathrm{M}_{\mathrm{vir}}<10^{7} \mathrm{M}_{\odot}$ in the B11_27 simulation, the reionization history does not deviate significantly from the other simulations, consistent with expectations from Sanati et al. (2020). Our stellar and dark matter particle mass resolution can suppress star formation in these low-mass haloes if they are underresolved. However, previous work using similar star formation and feedback models and a standard $\Lambda$ CDM primordial matter power spectrum shows that reionization is primarily driven by haloes with $\mathbf{M}_{\mathrm{vir}}>10^{8} \mathrm{M}_{\odot}$ (Kimm et al. 2017) and we therefore expect these results to hold even for higher resolution simulations. 


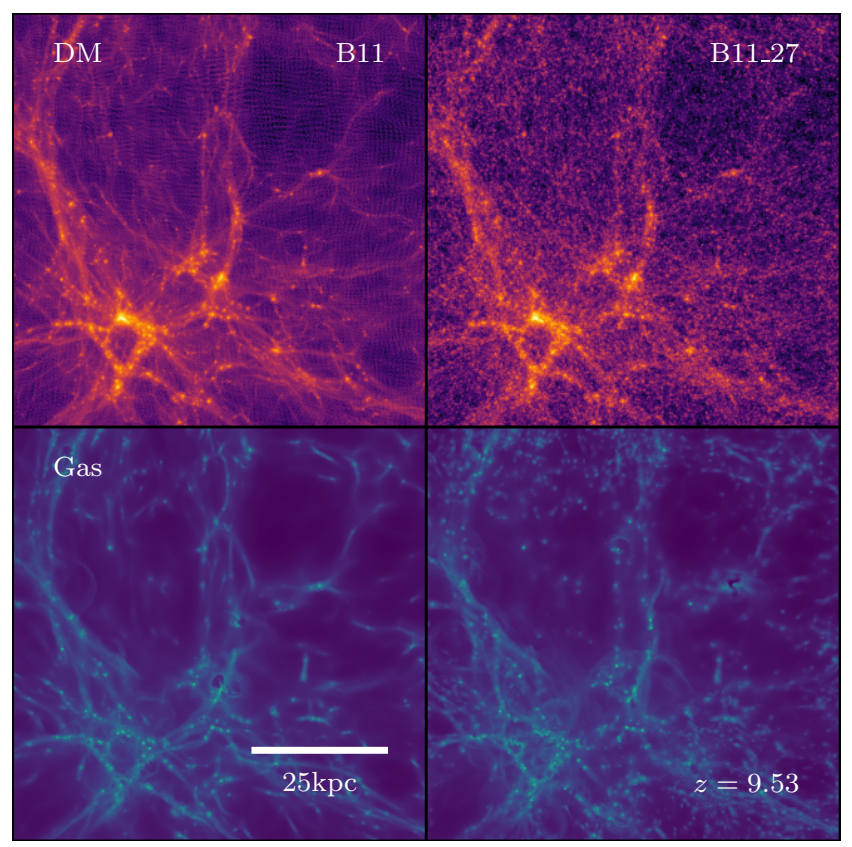

Figure 18. Surface density maps of dark matter (top) and gas (bottom) for the central $25 \%$ of the computation volume at $z=9.53$ for the B11 (left) and B11_27 (right) simulations. The enhancement in number density of low mass dark matter haloes can be seen in the B11_27 simulations and the baryon distribution is also clearly modified at this redshift.

In the bottom panel of Figure 16 we show the volume-weighted HI photoionisation rate $\left(\Gamma_{\mathrm{HI}}\right)$ in regions that are more than $50 \%$ ionised as a function of redshift. Once again, we find good agreement between the simulations, as expected given their consistent reionization histories. Many of the small fluctuations in $\Gamma_{\mathrm{HI}}$ correlate between the simulations. These are indicative of individual haloes collapsing or galaxy mergers resulting in large bursts of star formation that temporarily dominate $\Gamma_{\mathrm{HI}}$. For larger volume simulations, the evolution of $\Gamma_{\mathrm{HI}}$ would be much more smooth. Consistent with other work (e.g. Katz et al. 2018; Rosdahl et al. 2018), after the formation of the first stars, $\Gamma_{\mathrm{HI}}$ is high as the ionised regions only consist of the volume very close to galaxies. As the HII regions expand into the IGM, $\Gamma_{\mathrm{HI}}$ decreases. Once the HII bubbles begin to merge, percolation occurs and $\Gamma_{\mathrm{HI}}$ begins to increase again.

By $z=6$, the three simulations with the lowest neutral fractions (B11, B11_29, and B11_27) have photoionisation rates consistent with observations, while the B14 and B21 simulations exhibit a $\Gamma_{\mathrm{HI}}$ that is approximately five times weaker. $\Gamma_{\mathrm{HI}}$ is expected to rapidly increase right at the end of reionization, when there is significant overlap of ionised bubbles (see Figure 10 of Rosdahl et al. 2018), and thus such a difference is expected between simulations with mildly different reionization histories.

Due to the differences in initial matter power spectrum and the resulting modifications to the dark matter halo mass function, we might expect that the presence of PMFs may have a significant impact on the Ly $\alpha$ forest (e.g. Pandey \& Sethi 2013; Chongchitnan \& Meiksin 2014). In Figure 17 we show projected surface-density maps of the dark matter and gas distribution for the central $25 \%$ of the computational volume at $z=6$ for each of the simulations. Consistent with what was observed in the dark matter-only simulations, we can clearly see a strong enhancement in the number of dark matter clumps in the B11_27 simulation compared to any of the fiducial models that do not include any modification to the matter power spectrum. Most

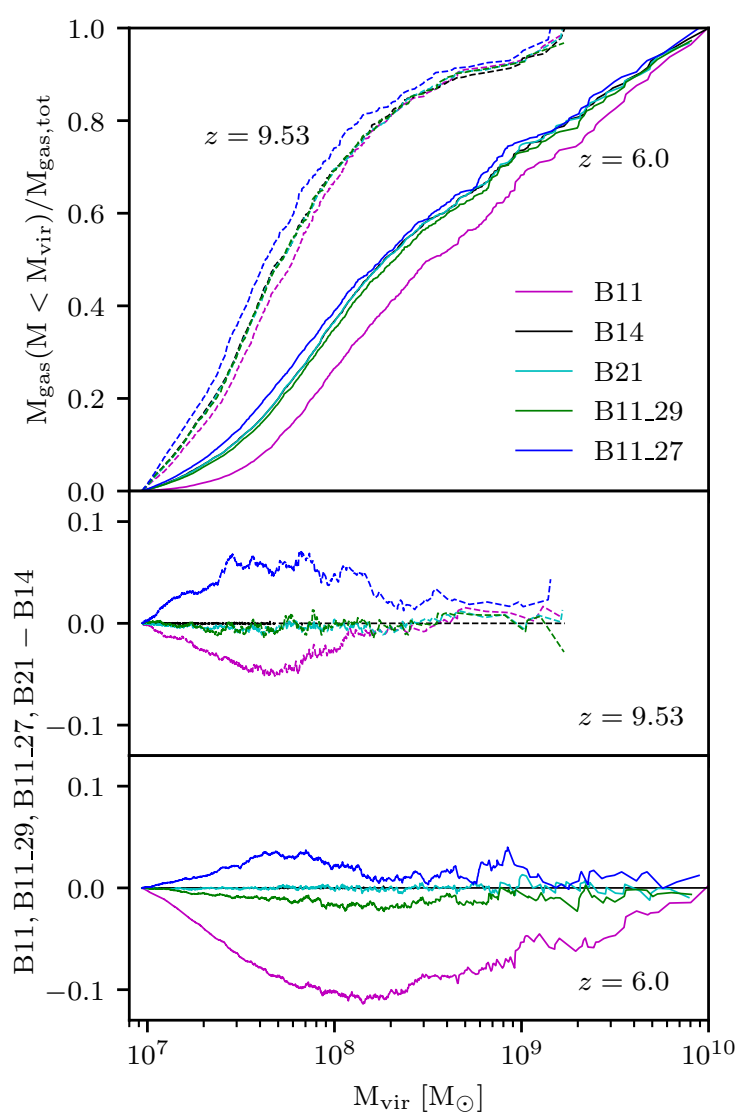

Figure 19. Fraction of the total gas mass in haloes that is contained in haloes with $\mathrm{M}<\mathrm{M}_{\mathrm{vir}}$ at $z=6$ (solid) and $z=9.53$ (dashed) for each of the simulations (top). We show the difference between each of the simulations and the B14 simulation for this quantity at $z=9.53$ (centre) and at $z=6$ (bottom). There is an excess in gas contained in low mass haloes in the B11_27 simulation compared to the $\mathrm{B} 14$ simulation while there is a reduction in the gas content of low mass galaxies in the B11 simulation.

noticeable is that the filamentary structure in the B11_27 simulation is significantly less smooth.

Despite the differences in the $z=6$ dark matter distribution, the gas distribution appears visually very similar in the bottom row of Figure 17, albeit small differences near galaxies where stellar feedback has clearly had an impact. These results are consistent with Pandey \& Sethi (2013) who require either stronger magnetic fields or larger spectral indices in order to obtain observable differences in the effective optical depth, some of which are inconsistent with the history of reionization (Sanati et al. 2020).

More quantitatively, we can compare the surface density distributions of gas and dark matter between the different simulations. For simulations without modified initial conditions, one would expect stronger differences in the gas compared to the dark matter due to feedback and other hydrodynamic processes. When compared to the B14 simulation the mean difference in the region shown in Figure 17 is $25 \%$ and $7.5 \%$ for the dark matter for the $\mathrm{B} 11$ and $\mathrm{B} 21$ simulations, respectively. It is not surprising that there is a larger deviation for the $\mathrm{B} 11$ simulation compared to the $\mathrm{B} 21$ simulation as the additional magnetic pressure from the strong PMF can impact the dark matter indirectly by modifying the baryon distribution. In contrast, we find mean deviations of 59\% and 54\% in the gas surface density for the B11 and B21 simulations, respectively. The situation is reversed for the B11_29 and B11_27 simulations. For the dark matter, we find a 


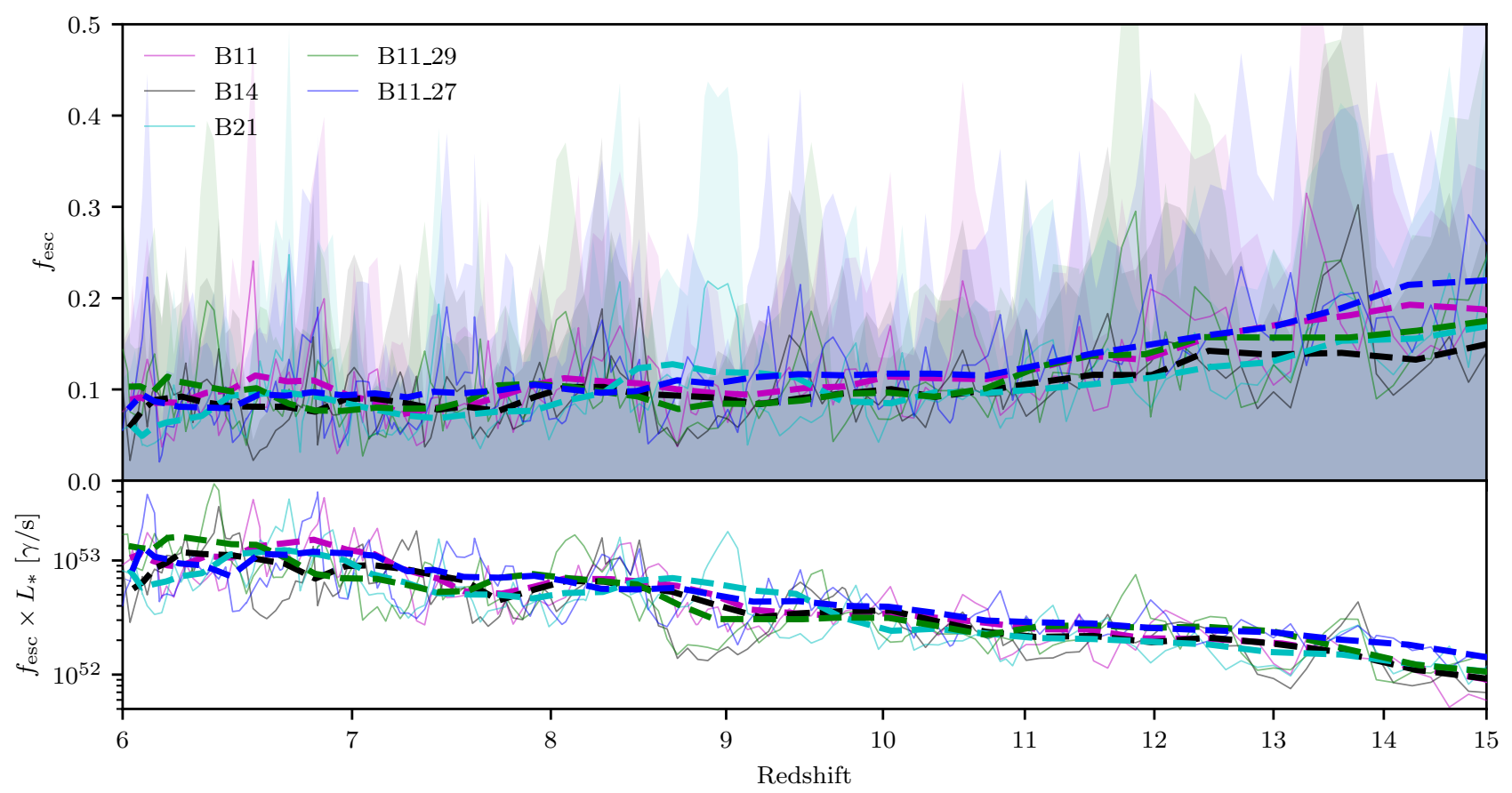

Figure 20. Luminosity-weighted mean escape fraction (top) and number of escaping ionising photons (bottom) as a function of redshift for each of the simulations. The solid lines and shaded region represent the mean and $1 \sigma$ standard deviation of $f_{\text {esc }}$ as measured at each individual snapshot. The dashed lines represent the luminosity-weighted average $f_{\text {esc }}$ over the previous $100 \mathrm{Myr}$.

difference of $880 \%$ and $1924 \%$ for the B11_29 and B11_27 simulations, respectively compared to the B14 simulation while for the gas, the difference is only $53 \%$ and $66 \%$, which is much more consistent with what we found for the B11 and B21 simulations.

For the B11_29 and B11_27 simulations, the modifications to the power spectrum only have a strong impact on haloes with $\mathrm{M}_{\mathrm{vir}} \lesssim 3 \times 10^{7} \mathrm{M}_{\odot}$ (see Figure 2 ). It is well established that photoionization and photoheating from the process of reionization can starve, photoevaporate, and prolong cooling times around low-mass dwarf galaxies (Rees 1986; Efstathiou 1992; Okamoto et al. 2008; Gnedin \& Kaurov 2014; Dawoodbhoy et al. 2018; Katz et al. 2020b), particularly those with virial temperatures below the atomic cooling threshold. Furthermore, reionization can reduce the gas masses of filaments by more than $80 \%$ (Katz et al. 2020b). Hence the structures that are most modified by the presence of the primordial magnetic fields sampled in this work are also those that are most sensitive to radiation feedback. If reionization is indeed the process that is smoothing the gas density field and erasing the small-scale structure, at higher redshifts, prior to reionization, we should observe differences in the gas density field. In Figure 18, we show the dark matter and gas density field in the same central region of the volume as Figure 17; however, here, we show the results at $z=9.53$ when the simulation volume is less than $20 \%$ ionised. Here we can see that there are significantly more small clumps of gas in the B11_27 simulation.

We illustrate this more quantitatively in Figure 19 where we show the fraction of the total gas mass in haloes that is contained in haloes with $\mathrm{M}<\mathrm{M}_{\text {vir }}$ at $z=6$ and $z=9.53$. At $z=9.53$, the B11_27 simulations exhibits a $\sim 7 \%$ excess in gas mass in haloes with $\mathrm{M}_{\mathrm{vir}}<3 \times 10^{7} \mathrm{M}_{\odot}$ compared to the B14, B21, and B11_29 sim- ulations. This excess decreases by more than a factor of two by $z=6$ where reionization has smoothed the density field. In contrast, the B11 simulation exhibits $\mathrm{a} \sim 5 \%$ and $\sim 10 \%$ reduction in the gas content of haloes with $\mathrm{M}_{\mathrm{vir}}<10^{8} \mathrm{M}_{\odot}$ at $z=9.53$ and $z=6$, respectively. The reduction at high redshift is likely due to the enhanced pressure support that may slow gas accretion. By $z=6$ the B11 simulation is more ionised than the other volumes and hence the difference grows. This effect can also be seen in the B11_29 simulation. At $z=9.53$, the cumulative distribution function of the B11_29 simulation is nearly identical to that of the B14 and B21 simulations. However, this simulation is more ionised at $z=6$ and hence there is a reduction in the gas content of low mass haloes compared to these other simulations.

\subsection{Impact of primordial magnetic fields on the LyC escape fraction}

Constraining the LyC escape fraction as a function of both redshift and galaxy properties has been the subject of numerous observational and theoretical studies as constraining this parameter will allow for the determination of both the reionization history and the primary sources responsible (e.g. Nakajima et al. 2020; Kimm et al. 2017; Barrow et al. 2020). While the global, luminosity-weighted escape fractions cannot be substantially different between the simulations with different PMFs because of the observed consistency in star formation and reionization histories, we may expect slight differences due to the variations in ISM properties.

In Figure 20 we show the luminosity-weighted $f_{\text {esc }}$ as a function of redshift for each of the simulations as well as the $1 \sigma$ scatter about the relation. The escape fractions are slightly higher at high redshifts 


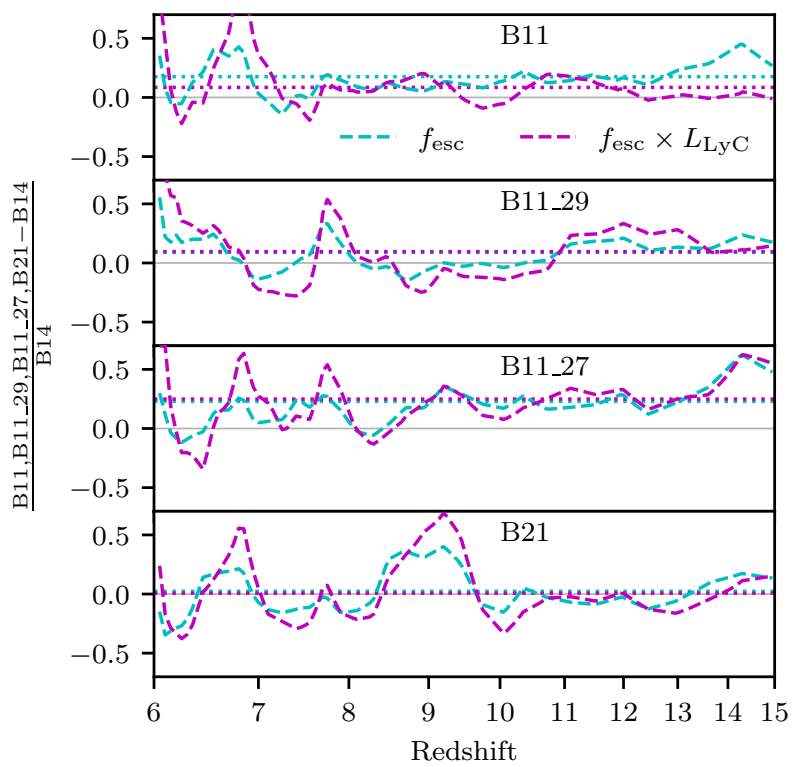

Figure 21. Fractional difference between the $100 \mathrm{Myr}$ averaged $f_{\text {esc }}$ (cyan) and the average of $f_{\mathrm{esc}} \times L_{\mathrm{LyC}}$ (magenta) in the B11 (top), B11_29 (second panel), B11_27 (third panel), and the B21 (bottom) simulations and the B14 simulation. The horizontal magenta and cyan lines represent the mean difference between $z=6$ and $z=15$. In general, the simulations with stronger PMFs exhibit higher LyC escape fractions as well as a greater number of leaking LyC photons but the effect is only 25\%, whereas the average escape fractions are nearly identical in the simulations with weaker PMFs.

where low-mass dwarf galaxies dominate the photon budget. We note that $f_{\text {esc }}$ is also a function of metallicity (Yoo et al. 2020) which evolves with redshift. $f_{\text {esc }}$ decreases as a function of redshift from $>15 \%$ at $z=15$ to $f_{\mathrm{esc}} \sim 5 \%-10 \%$ at $z=6$. The $z=6$ values for $f_{\text {esc }}$ in our simulations are in complete agreement with the larger volume non-MHD simulations of Rosdahl et al. (2018) that also employ a stellar SED that includes binary stars (Eldridge et al. 2008; Stanway et al. 2016).

As expected from the reionization histories, the simulations all exhibit luminosity-weighted escape fractions that are well within each others $1 \sigma$ standard deviation. Because the galaxies in the simulations with strong magnetic fields are more compact, we might expect small differences in $f_{\text {esc }}$ due to the changes to the ISM. In Figure 21 we show the fractional difference between the B11, B11_29, B11_27, and B21 simulations and the B14 simulation as the dashed cyan lines. The average values of $f_{\text {esc }}$ in the simulations with the stronger PMFs are $10 \%-25 \%$ higher in the B11 series simulations compared to $\mathrm{B} 14$ while the average value of $f_{\text {esc }}$ is nearly identical between B14 and B21. The enhancement is not particularly large (e.g. compared to the difference between single star and binary star SEDs which is upwards of $300 \%$ Rosdahl et al. 2018) and it also fluctuates. Because the density field is different in the B11_27 and B11_29 simulations, the cleanest comparison is between the B11 and B14/B21 simulations. There is a small difference in reionization history; however, the could also be due to small differences in the star formation history. LyC photon production is the other important variable in determining reionization history and thus in Figure 21, we also show a comparison of the $100 \mathrm{Myr}$ average $f_{\mathrm{esc}} \times L_{\mathrm{LyC}}$ where $L_{\mathrm{LyC}}$ is the LyC photon luminosity. We can see that the increase in escape fraction is not compensated by a decrease in LyC luminosity for the simulations
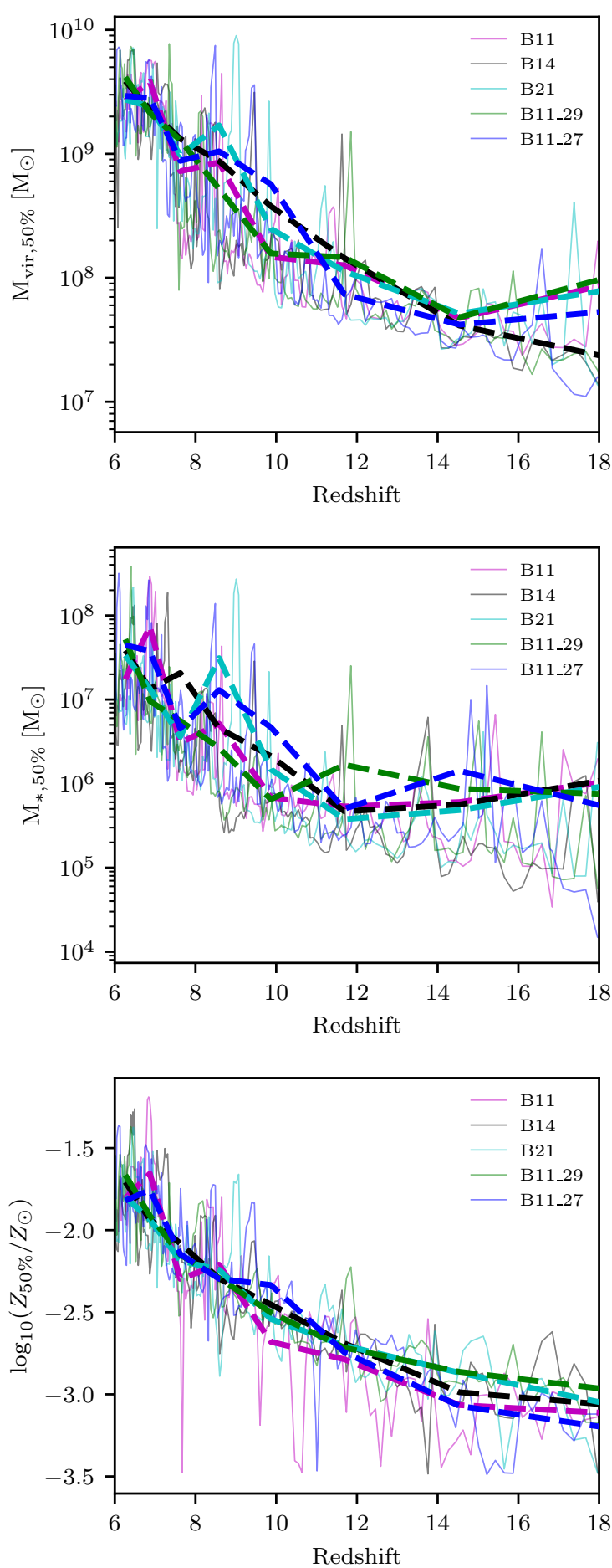

Figure 22. Halo mass (top), galaxy stellar mass (centre), and stellar metallicity (bottom) below which $50 \%$ of the leaking ionising photons originate as a function of redshift. For example, at $z=10,50 \%$ of the ionising photons that leak into the IGM are emitted by haloes with $\mathrm{M}_{\mathrm{vir}}<10^{8} \mathrm{M}_{\odot}$. Dashed lines represent the $100 \mathrm{Myr}$-averaged values of the individual quantities. As the universe evolves, most of the photons that reionize the Universe originate in more massive galaxies and more metal enriched stars. 


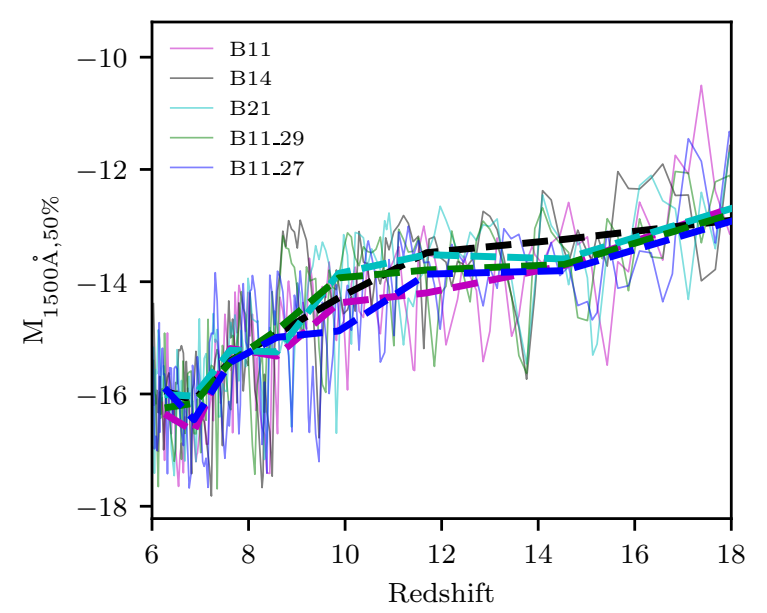

Figure 23. $1500 \AA$ absolute magnitude below which $50 \%$ of the leaking ionising photons originate as a function of redshift. For example, at $z=6$, $50 \%$ of the ionising photons that leak into the IGM are emitted by galaxies with UV magnitudes fainter than -16 . Dashed lines represent the 100Myraveraged values of $M_{1500 \AA, 50 \%}$.

with strong PMFs. This explains why the HI photoionization rates are higher in the B11, B11_29, and B11_27 simulations at $\mathrm{z}=6$ compared to the B14 and B21 simulations. We emphasise that this effect is small and therefore caution over-interpreting this result.

In Figure 22 we explore which sources are responsible for providing the photons that drive reionization as a function of redshift. For each simulation snapshot, we calculate the cumulative distribution function of the escaping ionising luminosity as a function of halo mass (top), galaxy stellar mass (centre), and stellar metallicity (bottom) and plot the 50th percentile as a function of redshift. In the top panel of Figure 22 we can see that at $z=18,50 \%$ of all of the escaping ionising photons are emitted by haloes with $\mathrm{M}_{\mathrm{vir}} \sim 3 \times 10^{7} \mathrm{M}_{\odot}$. This value increases to well above $10^{9} \mathrm{M}_{\odot}$ by $z=6$. Similarly, the stellar masses of the galaxies driving reionization increases from $\sim 10^{5} \mathrm{M}_{\odot}$ at $z=18$ to $>10^{7} \mathrm{M}_{\odot}$ at $z=6$. As the universe evolves and galaxies become more massive, the sources driving reionization also increase in mass.

The metallicity of the stars driving reionization also strongly increases with redshift. Even at $z=18$, the stars that are contributing most of the escaping photons to reionization have been enriched above our primordial metallicity floor of $10^{-3.5} Z_{\odot}$. This is consistent with $\mathrm{Xu}$ et al. (2016) who found that the contribution from Population III stars is expected to dominate at $z>16$.

We find no significant differences between the sources of reionization in any of the simulations with different PMFs at redshifts up to $z=18$. Note that out limited mass and spatial resolution prohibits us from self-consistently modelling the formation of Population III stars. Given the strong enhancement in the number of low mass galaxies in the B11_27 simulation, a higher resolution simulation may find some differences at $z>16$ where these haloes are expected to dominate.

A particularly interesting question is whether we can observe the sources that are driving reionization. In Figure 23, we plot the intrinsic $1500 \AA$ absolute UV magnitude below which $50 \%$ of the escaping ionising photons originate as a function of redshift. We find that this magnitude decreases from -12 at $z=18$ to $\sim-16$ at $z=6$, consistent with Wise et al. (2014). Using strong lensing from galaxy clusters, the Hubble Frontier Fields can already probe UV magni- tudes much fainter than -16 at $z=6$ (e.g. Livermore et al. 2017) and thus, current observations have already detected the galaxies that are primarily responsible for keeping the Universe reionized at $z=6$. Because the photoionization and photoheating from reionization are incredibly efficient at suppressing star formation in low mass dwarf galaxies at halo masses that we resolve (Okamoto et al. 2008; Xu et al. 2016; Katz et al. 2020b), this result is expected to hold even for higher resolution simulations. Furthermore, larger boxes will only probe brighter galaxies which could only bias our measurements in Figure 23 towards brighter magnitudes (see Lewis et al. 2020), hence strengthening our argument.

\subsection{Constraints on the properties of primordial magnetic fields}

The properties for the PMFs chosen in this work bracket the range of having no impact on the history of reionization to having an ionised fraction of $50 \%$ at $z>50$. Due to computational resource limitations, we cannot conduct radiation magnetohydrodynamics simulations for an entire grid of values of $B_{0}$ and $n_{B}$. In this section, we construct analytic models based on the results of our simulations to improve our constraints on the properties of the PMF.

\subsubsection{Ionisation history and electron optical depth constraints}

The evolution of the ionised hydrogen fraction $\left(Q_{\mathrm{HII}}\right)$ can be modelled using the following ordinary differential equation:

$\frac{d Q_{\mathrm{HII}}}{d t}=\frac{\dot{n}_{\text {ion }}}{\left\langle n_{\mathrm{H}}\right\rangle}-\frac{Q_{\mathrm{HII}}}{\bar{t}_{\mathrm{rec}}\left(C_{\mathrm{HII}}\right)}$,

(e.g. Madau et al. 1999), where $\dot{n}_{\text {ion }}$ is the ionising photon production rate $\left(\gamma / \mathrm{s} / \mathrm{cMpc}^{3}\right),\left\langle n_{\mathrm{H}}\right\rangle$ is the mean comoving hydrogen number density, $C_{\mathrm{HII}}$ is the clumping factor of ionised hydrogen, and $\bar{t}_{\mathrm{rec}}$ is the volume-averaged recombination timescale of HII. $\bar{t}_{\text {rec }}$ is a function of temperature and the clumping factor such that

$\bar{t}_{\mathrm{rec}}=\frac{1}{C_{\mathrm{HII}} \alpha_{\mathrm{B}}(T)\left(1+\frac{Y}{4 X}\right)\left\langle n_{\mathrm{H}}\right\rangle(1+z)^{3}}$

(e.g. Kuhlen \& Faucher-Giguère 2012), where $\alpha_{\mathrm{B}}(T)$ is the temperature-dependent case-B HII recombination rate (Hui \& Gnedin 1997), $Y$ is the primordial helium mass fraction, and $X$ is the primordial hydrogen mass fraction. We choose a fixed value of $2 \times 10^{4} \mathrm{~K}$ for the IGM temperature ${ }^{9}$ (e.g. Trac et al. 2008) and to be consistent with our simulations, we set $X=0.76$ and $Y=0.24$. For the clumping factor, we follow Kimm et al. (2017) and adopt a redshift-dependent value of $C_{\mathrm{HII}}=1+e^{-0.28 z+3.59}$ at $z \geq 10$ and $C_{\mathrm{HII}}=3.2$ at $z<10$ (Pawlik et al. 2009). We note that the values of $Q_{\text {HII }}$ at high redshifts are especially sensitive to this parameter.

$\dot{n}_{\text {ion }}$ as a function of redshift can be modelled as

$\dot{n}_{\text {ion }}=\xi_{\text {ion }}(z) f_{\text {esc }}(z) \rho_{\text {SFR }}(z)$,

where $\rho_{\mathrm{SFR}}(z)$ is the star formation rate density as a function of redshift, $f_{\text {esc }}(z)$ is the escape fraction of ionising photons as a function of redshift, and $\xi_{\text {ion }}(z)$ is the ionising photon production efficiency per stellar mass as a function of redshift. To obtain the star formation rate density, we first generate the matter power spectrum for each PMF for different values of $n_{B}$ and $B_{0}$ using the modified version of CAMB (Shaw \& Lewis 2012). We then use COLOSSUS (Diemer

9 Note that this temperature is consistent with the local reionization temperature of the SPHINX simulations. See Figures 9 and 10 of Katz et al. (2020b). 
2018) to calculate the redshift-dependent halo mass function (Press $\&$ Schechter 1974). Each halo is populated with stars using the stellar mass-halo mass relation from our B11 simulation as a function of redshift. Note that this function evolves only mildly with redshift up to $z \sim 20$. We extrapolate the function to both lower and higher mass haloes than what are resolved or sampled by our simulation and additional systematic uncertainty may result from this extrapolation. Not all low mass haloes host stellar populations (i.e. the occupation fraction, $f_{\text {occ }}$, is less than 1). To account for this, we use the fitting formula for the occupation fraction based on the SPHINX simulations (Katz et al. 2020b),

$f_{\mathrm{occ}}=\frac{1}{1+e^{-k\left[\log _{10}\left(\mathrm{M}_{\mathrm{vir}}\right)-\mathrm{M}_{\mathrm{c}}(z)\right]}}$,

where $k=8.20$ and $\mathrm{M}_{\mathrm{c}}(z)=-0.05 c+8.32$. The total star formation rate per unit volume is measured as the growth in total stellar mass as a function of redshift. $f_{\text {esc }}(z)$ is calculated using a linear fit to the redshift evolution of the luminosity-weighted $f_{\text {esc }}$ in the B11 simulation such that

$f_{\mathrm{esc}}(z)=\operatorname{Min}(0.0096 z+0.0246,50 \%)$.

We have capped $f_{\text {esc }}$ to prevent it from reaching unrealistic values at very high redshifts such that the value is consistent with the escape fractions measured in simulated mini-haloes (Wise et al. 2014; Xu et al. 2016; Kimm et al. 2017). Note that we apply the escape fraction to the total $\rho_{\mathrm{SFR}}(z)$ rather than that of each halo mass as the luminosity-weighted value of $f_{\text {esc }}$ should capture any mass dependence. Furthermore, the SPHINX simulations do not show a strong evolution of $f_{\mathrm{esc}}$ with halo mass (Rosdahl et al. in prep). Finally, the value of $\xi_{\text {ion }}$ is fixed to $10^{53.36} \mathrm{\gamma} / \mathrm{s} /\left(\mathrm{M}_{\odot} / \mathrm{yr}\right)$, consistent with the lowest metallicity bin of the BPASSv2.0 SED that was used in the simulations. From the bottom panel of Figure 22, we can see that most of the stars that provide ionising photons have metallicities lower than the lowest metallicity bin of the BPASSv2.0 SED. Therefore, we do not apply a redshift dependence to this value.

In the top panel of Figure 24, we plot $\dot{n}_{\text {ion }}$ as a function of redshift for PMFs with $B_{0}=0.05 \mathrm{n} G$ and $n_{B}$ in the range [-3.0,-2.3]. The grey line which represents $n_{B}=-3.0$ reproduces the results from the B11 simulation confirming that our analytic model is well calibrated with the simulation.

As $n_{B}$ increases, $\dot{n}_{\text {ion }}$ deviates more from the fiducial case of $n_{B}=-3.0$. Modifications to the matter power spectrum drive earlier collapse of more massive haloes. Extreme models (e.g. $n_{B}=-2.4$ ) have a decreasing $\dot{n}_{\text {ion }}$ as a function of redshift. The shapes of these lines are dictated by the competition between the collapse of new haloes and the decreasing occupation fraction of low mass haloes as a function of redshift. Flatter values of $n_{B}$ result in enhancements in the number densities of higher mass haloes as well (see Figure 2) which are only impacted by the evolving occupation fraction at lower redshifts.

In the centre panel of Figure 24, we show $Q_{\mathrm{HII}}$ as a function of redshift for $B_{0}=0.05 \mathrm{n} G$ and $n_{B}$ in the range [-3.0,-2.3]. For comparison, we also show $Q_{\mathrm{HII}}$ as a function of redshift for the B11 (dashed), B11_27 (solid), and B11_24 simulations (dotted). As expected, the model with $n_{B}=-3.0$ reionizes at $z \sim 6$, consistent with the B11 simulation. Similarly, the model with $n_{B}=-2.7$ closely follows the model with $n_{B}=-3.0$ and exhibits a reionization history consistent with the B11_27 simulation. The model with $n_{B}=-2.4$ exhibits an ionisation fraction of $40 \%$ at $z=50$, which is consistent with the early evolution of $Q_{\text {HII }}$ in the B11_24 simulation; however, the analytic model exhibits a more gradual evolution in $Q_{\mathrm{HII}}$ compared to the simulation. This may be due to the fact that the
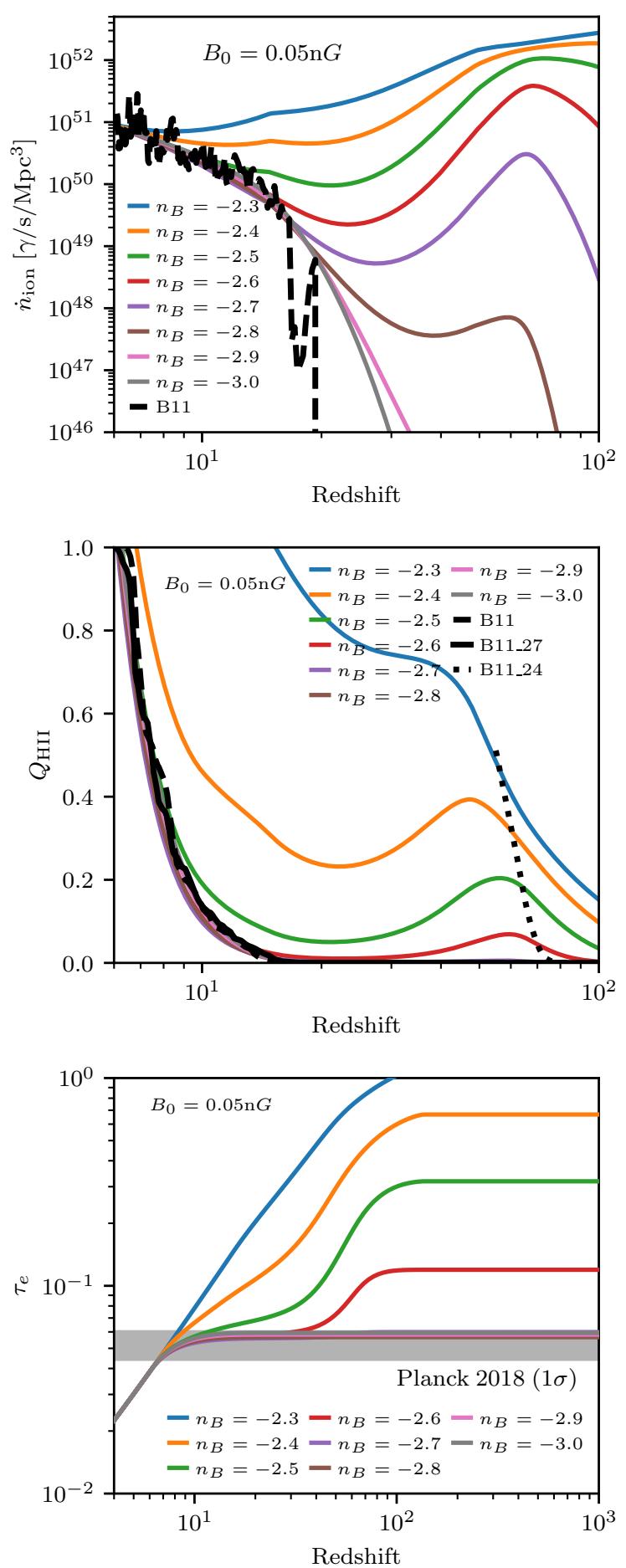

Figure 24. (top) Ionising photon production rate, $\dot{n}_{\text {ion }}$, as a function of redshift for PMF models with $B_{0}=0.05 \mathrm{n} G$ and $n_{B}$ in the range $[-3.0,-2.3]$. The dashed black line shows the results from the B11 simulation. (centre) Ionised fraction $Q_{\mathrm{HII}}$ as a function of redshift for each PMF model compared to the results from the B11 (dashed), B11_27 (solid), and B11_24 (dotted) simulations represented by black lines. (bottom) Electron optical depth for each PMF compared to the constraints from Planck Collaboration et al. (2018). 


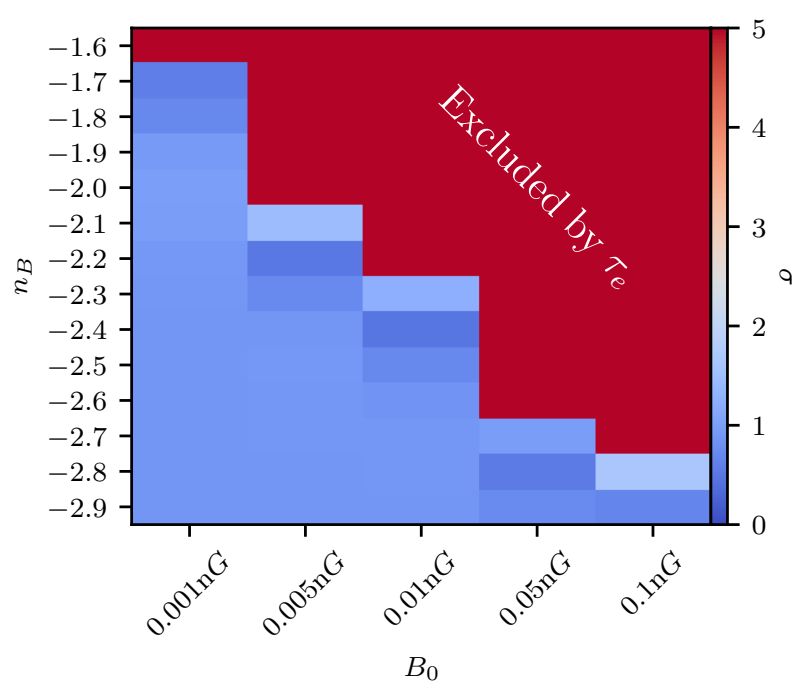

Figure 25. Constraints on $n_{B}$ as a function of $B_{0}$ based on the electron optical depth $\tau_{e}$. The colour at each grid point indicates by how many $\sigma=$ $\left|\tau_{e \text {, model }}-\tau_{e \text {,Planck }}\right| / \sigma_{\tau_{e} \text {,Planck }}$ the measured value of $\tau_{e}$ deviates from the Planck Collaboration et al. (2018) value.

simulation does not have high enough resolution to resolve some of the low mass haloes that may be contributing ionising photons at these redshifts. Furthermore, the recombination timescales at high redshift are very short and we note again that $Q_{\mathrm{HII}}$ at these high redshifts is very sensitive to the chosen clumping factor. The final reionization redshift for the model with $n_{B}=-2.4$ is only slightly higher than that of the other models because the short recombination timescale and the evolving occupation fraction result in a decreasing $\dot{n}_{\text {ion }}$. Unfortunately, computational limitations prevent us from evolving the B11_24 simulation to the redshifts where the analytic models predict a turnover in $Q_{\mathrm{HII}}$. For $n_{B}=-2.3$, the reionization redshift is completely incompatible with observations as it occurs at $z \sim 15$. Our results are once again qualitatively consistent with the analytic models of (Sanati et al. 2020) who demonstrated that strong differences in the reionization history only appear for models with $n_{B} \gtrsim-2.4$.

For models with $B_{0}=0.05 \mathrm{n} G$ and $n_{B} \geq-2.6, Q_{\mathrm{HII}}$ deviates noticeably from zero at very high redshift due to early onset star formation. Although $Q_{\mathrm{HII}}$ decreases again due to the short recombination timescale and the evolution of $f_{\text {occ }}$, the free electrons at high redshift can impact the electron optical depth, $\tau_{e}$. In the bottom panel of Figure 24, we plot $\tau_{e}$ for each of the PMF models. While the models with $n_{B}<-2.6$ have a $\tau_{e}$ that is consistent with observations of the CMB (Planck Collaboration et al. 2018), flatter spectral indices result in electron optical depths that are inconsistent with observational constraints. Hence $\tau_{e}$ places a stronger constraint on the properties of PMFs compared to observations of the reionization history.

Using our calibrated model, we run a grid of calculations varying both $B_{0}$ and $n_{B}$ and use $\tau_{e}$ to put constraints on the allowable values of $n_{B}$ for a given $B_{0}$. In Figure 25 we plot $\sigma=$ $\left|\tau_{e \text {, model }}-\tau_{e \text {,Planck }}\right| / \sigma_{\tau_{e \text {,Planck }}}$ for each of the models in the grid. As the value of $B_{0}$ decreases, the allowable values of $n_{B}$ can deviate more from the scale-free case. At $B_{0}<10^{-12} G$, we have exhausted our grid and all $n_{B}<-1.6$ are in the allowed region. The boundary for allowable values of $n_{B}$ as a function of $B_{0}$ is nicely fit by the

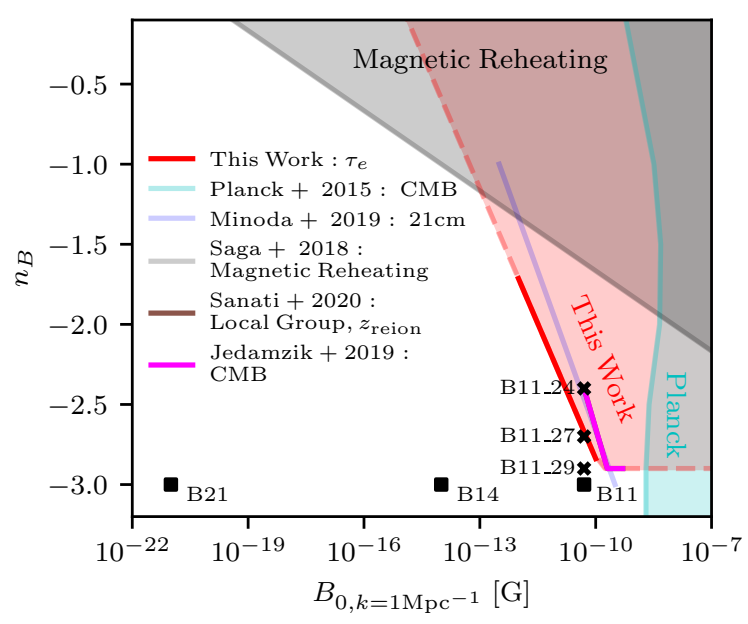

Figure 26. Constraints on $n_{B}$ as a function of $B_{0}$ from $\tau_{e}$ derived in this work (red) compared to other constraints from the literature. The shaded regions represents $B_{0}, n_{B}$ combinations that are excluded. The dashed red line shows where our constraints have been extrapolated. For comparison, we show constraints from Planck Collaboration et al. (2016) from observations of the CMB (cyan), from Saga et al. (2018) based on changes to the baryonphoton number ratio resulting from heating caused by the dissipation of PMFs on small scales (magnetic reheating, grey), from Jedamzik \& Saveliev (2019) from CMB anisotropies, and from Sanati et al. (2020) due to the impact of PMFs on the reionization history and the number of luminous local group dwarf galaxies (brown). We also show constraints from Minoda et al. (2019) based on the impact of heating from ambipolar diffusion and decaying magnetic turbulence (blue) on the $21 \mathrm{~cm}$ signal. Note that these constraints only apply if the signal observed by EDGES Bowman et al. (2018) is confirmed. Data points represent the parameters of our numerical simulations. "X's" show the locations of the B11_29, B11_24, and B11_24 and squares represent the B21, B14, and B11 simulations. We have placed the latter three simulations at $n_{B}=-3.0$ for visualisation purposes. because there is no impact to the matter power spectrum, but we stress that these simulations have been initialised with a uniform magnetic field.

relation:

$n_{B, \text { allowed }} \leq-0.562 \log _{10}\left(\frac{B_{0}}{\ln G}\right)-3.35$.

In Figure 26 we compare our constraints on the properties of the PMFs based on $\tau_{e}$ to others in the literature. In general, our constraints are tighter than those based on spectral distortions and other effects that PMFs have on the CMB (Planck Collaboration et al. 2016). Similarly, our results constrain a different part of $B_{0}-n_{B}$ space compared with Saga et al. (2018) who studied the impact of PMFs on the baryon-photon ratio. As stated previously, our constraints are consistent with those of Sanati et al. (2020) due to the impact of PMFs on the reionization history and the number of luminous local group dwarf galaxies. Finally, our constraint runs nearly parallel to but slightly stronger than that of Minoda et al. (2019) who used the EDGES signal (Bowman et al. 2018) to constrain the properties of PMFs based on the heating from ambipolar diffusion and decaying magnetic turbulence (see Section 3.6.2).

We emphasise that these analytic models are subject to limitations. We have had to extrapolate certain relations (e.g. the occupation fraction and the stellar mass-halo mass relation) to redshifts that are not probed by our suite of simulations. Furthermore, the ionisation fraction at high redshift is sensitive to the assumptions that go into calculating the recombination timescale. This may differ in simulations with modified density fields. For strong enough PMFs, 
gas accretion onto low mass dwarf galaxies may be affected, which will impact our estimate of the star formation rate density. Similarly, the star formation histories of galaxies in our simulation tend to be bursty whereas our model implicitly assumes that they are smooth. Given that the analytic model is able to reproduce both $\dot{n}_{\text {ion }}$ and $Q_{\mathrm{HII}}$ for the B11, B11_29, and B11_27 simulation, we are confident that the model produces reasonable results.

\subsubsection{Constraints from the high-redshift global $21 \mathrm{~cm}$ signal}

Observations of the $21 \mathrm{~cm}$ line at high redshift are one of the most promising probes of the thermal history of the early Universe (e.g. Pritchard \& Loeb 2012). The brightness temperature of the signal relative to the $\mathrm{CMB}$ is

$\Delta T_{b}=27 Q_{\mathrm{HI}}(1+\delta)\left(\frac{\Omega_{b} h^{2}}{0.023}\right)\left(\frac{0.016}{\Omega_{m} h^{2}}\right)\left(\frac{1+z}{10}\right)^{0.5}\left(1-\frac{T_{\gamma}}{T_{s}}\right) \mathrm{mK}$,

where $Q_{\mathrm{HI}}$ is the neutral fraction, $\delta$ is the fractional overdensity, $T_{S}$ is the spin temperature of the gas, and $T_{\gamma}$ is the CMB radiation temperature (Furlanetto 2006). The standard picture for the signal usually follows that at $200 \lesssim z \lesssim 1100$, there is no signal as the gas kinetic temperature and spin temperature are coupled to the radiation temperature due to Compton scattering. As the Universe expands, the gas cools adiabatically and $T_{S}$ drops below $T_{\gamma}$ due to collisional coupling, hence the signal is seen in absorption. When the density drops below a certain value, collisional coupling becomes inefficient and radiative coupling sets $T_{s} \sim T_{\gamma}$, diminishing the signal. After the formation of the first stars (and possibly black holes), Ly $\alpha$ and X-ray photons once again couple $T_{s}$ to the gas temperature which remains below $T_{\gamma}$, resulting in a second absorption signal. Eventually heating from reionization raises the gas above $T_{\gamma}$ and the $21 \mathrm{~cm}$ signal can be seen in emission until reionization completes (Pritchard \& Loeb 2012).

The presence of primordial magnetic fields can disrupt this standard model in numerous ways. As discussed in Section 2.2.3, ambipolar diffusion an decaying magnetic turbulence can increase the ionisation fraction and temperature of the IGM early on in the evolution of the Universe. These two effects are well studied in the literature (e.g. Tashiro \& Sugiyama 2006; Sethi \& Subramanian 2009; Schleicher et al. 2009). Because of these effects, for strong enough PMFs, $T_{S}$ never drops below $T_{\gamma}$ and the $21 \mathrm{~cm}$ signal can only be seen in emission (Sethi \& Subramanian 2009). This is in possible conflict with the recent observations from EDGES (Bowman et al. 2018) that exhibit a strong absorption profile in the range $14 \lesssim z \lesssim 22$. Such results indeed place strong constraints on the properties of PMFs (e.g. Minoda et al. 2019; Bera et al. 2020; Natwariya 2020).

While the heating from ambipolar diffusion and decaying magnetic turbulence is well studied, the impact on the global $21 \mathrm{~cm}$ signal from the modification to structure formation due to PMFs has been less explored. In this section, we employ an analytic model for the global $21 \mathrm{~cm}$ signal to study how the modification to the matter power spectrum from PMFs impact the global $21 \mathrm{~cm}$ signal.

To compute the global $21 \mathrm{~cm}$ signal, we use the ARES code ${ }^{10}$ (Mirocha 2014, 2020) and calibrate the parameters of the models to the results from our simulations. The key components of the model are determining the evolution of the radiation temperature $\left(T_{\gamma}\right)$, spin temperature $\left(T_{S}\right)$, and gas kinetic temperature $\left(T_{K}\right) . T_{\gamma}$ is set to follow the CMB (i.e. $\left.T_{\gamma}=2.725(1+z)\right) . T_{K}$ is calculated via

$\frac{3}{2} \frac{d}{d t}\left(\frac{k_{B} T_{k} n}{\mu}\right)=\epsilon_{X}+\epsilon_{B}+\epsilon_{\text {compton }}+\Lambda_{C}$,

where $n$ is the gas density, $\epsilon_{X}$ is the photoionisation heating rate, $\epsilon_{\mathrm{B}}$ is the heating rate due to ambipolar diffusion and decaying magnetic turbulence, $\epsilon_{\text {compton }}$ is the compton heating rate, and $\Lambda_{C}$ is the cooling rate that encapsulates adiabatic cooling, collisional ionisation cooling, recombination cooling, and collisional excitation cooling (Fukugita \& Kawasaki 1994). In order to calculate $T_{K}$ (as well as $T_{s}$ and $\Delta T_{b}$ ), we need to know the emissivity and heating rates from astrophysical sources and derive the ionisation fraction as a function of redshift. For simplicity, we only consider the impact of Ly $\alpha$, HI-ionising, and X-ray photons in our calculation. We have modified ARES to include the heating rates for ambipolar diffusion and decaying magnetic turbulence following the equations presented in the Appendix of Chluba et al. (2015).

For each value of $B_{0}$ and $n_{B}$, we provide ARES the values of $\dot{n}_{\text {ion }}(z)$ computed in the previous section. Similarly, we also provide ARES with the number of photons between the Lyman limit and Ly $\alpha$ wavelengths as a function of redshift which are used to compute the Ly $\alpha$ flux. These values were found by integrating the lowest metallicity bin of the BPASSv2.0 SED and assuming an escape fraction of 1.0 at these wavelengths. The X-ray luminosity density is parametrised by the star formation rate density $\dot{\rho}_{*}(z)$ such that $L_{X}=f_{X} c_{X} \dot{\rho}_{*}(z)$, where $c_{X}=2.6 \times 10^{39} \mathrm{erg} \mathrm{s}^{-1}$ (Mineo et al. 2012) and $f_{X}=1$ is a scale factor for the relation ${ }^{11}$. We assume that $20 \%$ of the X-ray luminosity is deposited as heat into the IGM (Shull \& van Steenberg 1985; Mirocha et al. 2013) and that X-rays also contribute to the ionisation fraction of the gas via secondary ionisations (Shull $\&$ van Steenberg 1985). For consistency with the previous section, we have applied the same clumping factor evolution, assumed that each ionisation heats the gas to $2 \times 10^{4} \mathrm{~K}$, ignored the contribution from helium, and have edited the recombination rates in ARES to be consistent with the values in RAMSES-RT. With these assumptions, we have confirmed that the reionization histories calculated by ARES are consistent with those in our simulations and analytic models.

The spin temperature of neutral hydrogen is set such that

$T_{s}^{-1}=\frac{T_{\gamma}^{-1}+x_{\alpha} T_{\alpha}^{-1}+x_{c} T_{K}^{-1}}{1+x_{a}+x_{c}}$,

where $T_{\alpha} \approx T_{K}$ is the colour temperature of the gas and $x_{c}$ and $x_{\alpha}$ represent the collisional and radiative coupling coefficients, respectively. $x_{c}$ is computed by interpolating tabulated values from Zygelman (2005) and coupling due to the Wouthuysen-Field effect (Wouthuysen 1952; Field 1958) is computed following Hirata (2006) as

$x_{\alpha}=\frac{8 \pi \lambda_{\mathrm{Ly} \alpha}^{2} \gamma T_{*}}{9 A_{10} T_{\gamma}} S_{\alpha} J_{\alpha}$,

where $\lambda_{\mathrm{Ly} \alpha}$ is the $\operatorname{Ly} \alpha$ wavelength, $T_{*}=0.068 \mathrm{~K}$ is the temperature difference between the HI hyperfine states, $A_{10}=2.85 \times 10^{-15}$ is the $21 \mathrm{~cm}$ spontaneous emission coefficient, $\gamma=50 \mathrm{MHz}$ is the halfwidth at half maximum of the Ly $\alpha$ resonance, $J_{\alpha}$ is the flux of Ly $\alpha$ photons as computed earlier, and $S_{\alpha}$ is a suppression factor that accounts for radiative transfer effects near line centre of the Ly $\alpha$ line 

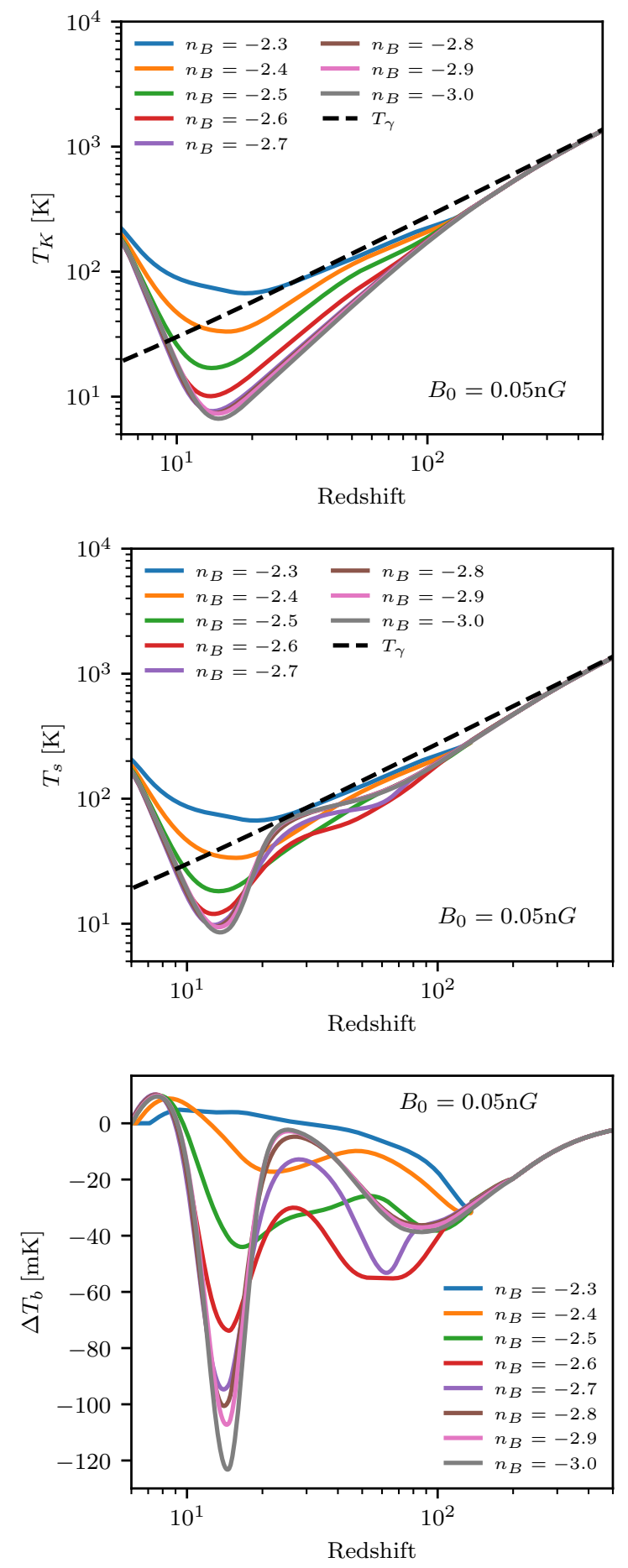

Figure 27. Gas kinetic temperature (top), spin temperature (centre) and global $21 \mathrm{~cm}$ signal (bottom) as a function of redshift for $B_{0}=0.05 \mathrm{n} G$ and various values of $n_{B}$. The dashed black line in the top and centre panels represent the evolution of the radiation temperature, $T_{\gamma}$.

(e.g. Furlanetto et al. 2006). We use the approximation for $S_{\alpha}$ given in Hirata (2006).

In Figure 27, we plot $T_{K}$ (top), $T_{S}$ (centre), and the global $21 \mathrm{~cm}$ signal (bottom) as a function of redshift for $B_{0}=0.05 \mathrm{n} G$ and various values of $n_{B}$. At $200 \lesssim z \lesssim 300$, no stars have formed in any of the models and thus $T_{S}$ and $T_{K}$ drop below $T_{\gamma}$, regardless of $n_{B}$ due to adiabatic expansion and collisional coupling. By $z \sim 150$, the first sources have formed in our most extreme models. This affects the signal in two ways. For models where the ionisation fraction becomes substantial (e.g. $n_{B} \geq-2.4$ ), the gas becomes hotter and quickly approaches $T_{\gamma}$. This depletes the strength of the first absorption feature expected at high redshifts. In contrast, for $n_{B}=-2.6$ or -2.7 , the heating is not so efficient that the absorption feature is erased, rather, radiative coupling sets in earlier and the spin temperature is coupled to the kinetic temperature for a longer redshift interval with leads to a larger and deeper absorption feature at high redshift (compare the red line with the grey line in the bottom panel of Figure 27). In both of these models, the spin temperature never fully couples back to the radiation temperature and the global $21 \mathrm{~cm}$ signal can always be seen in absorption until reionization fully sets in. However, excess heating in the models with $n_{B} \geq-2.6$ weakens the depth of the second absorption trough to the point where, when $n_{B}=-2.3$, the second absorption feature is absent and the signal can only be seen in emission.

We reiterate that none of the models with $n_{B} \geq-2.6$ are consistent with $\tau_{e}$ and thus the effects from these models on the global $21 \mathrm{~cm}$ are unlikely to hold. In contrast, the B11_27 simulation exhibits a reionization history and $\tau_{e}$ that are consistent with observational constraints while the $21 \mathrm{~cm}$ signal, in particular the first absorption feature, is strongly affected. For values of $-3.0<n_{B} \leq-2.7$, depth of the second absorption feature is only mildly reduced as $n_{B}$ increases. However, this behaviour is opposite to what is needed to be compatible with the EDGES signal.

The deep absorption feature seen by EDGES is already in conflict with predictions from standard astrophysical scenarios (Bowman et al. 2018). Numerous solutions have been proposed in order to explain the depth of the signal such as more exotic physics, for example, baryon-dark matter interactions or deviations from CDM (e.g. Barkana 2018; Muñoz \& Loeb 2018), an excess radio background (Fialkov \& Barkana 2019), or an enhanced Ly $\alpha$ background (e.g. Meiksin \& Madau 2020; Mittal \& Kulkarni 2020). It is also possible that the EDGES signal is entirely spurious (Hills et al. 2018). Unless the PMF is strong enough to prevent accretion onto low mass dwarf galaxies and inhibit star formation in the first collapsing haloes, the presence of strong PMFs with flatter spectral indices will reduce the depth of the global $21 \mathrm{~cm}$ signal and perhaps result in emission across the redshift range of the EDGES result. Should the EDGES result be validated, this would be indicative of either PMFs that have weak amplitudes or close to scale-free spectral indices, or other physics must counterbalance the impact of the early structure formation.

We note that the global $21 \mathrm{~cm}$ signal is sensitive to the chosen cosmological parameters in addition to the astrophysical modelling at high redshift. Our models have neglected effects such as baryon streaming velocities (Tseliakhovich \& Hirata 2010) which can reduce both the number density and gas content of early mini-haloes (e.g. $\mathrm{Naoz}$ et al. 2012, 2013). For large enough streaming velocities, the reduction in gas content and halo number density of low mass haloes may oppose the impact of the modification to the power spectrum, allowing for flatter a $n_{B}$ at a given $B_{0}$.

\section{DISCUSSION \& CONCLUSIONS}

We have presented the first results from the SPHINX-MHD simulations, a suite of cosmological radiation-magnetohydrodynamics simulations designed to study the impact of primordial magnetic fields on reionization and the formation of the first galaxies. We employ two different models for the properties of the PMFs: the first assumes 
a uniform magnetic field of a given strength as is common technique in cosmological MHD simulations (e.g. Pillepich et al. 2018) and the second assumes Gaussian random magnetic fields with a given strength and spectral index. The former is useful for understanding the impact of magnetic fields on the ISM while the latter has been used to study how the modifications to the matter power spectrum induced by the PMFs (Shaw \& Lewis 2012) impacts the history of reionization.

All of our simulations apart from the B11_24 simulation result in a $\tau_{e}$ that is consistent with Planck Collaboration et al. (2018). This parameter provides the tightest constraint on the properties of PMFs in the space of $B_{0}-n_{B}$ that we have sampled. For PMFs that satisfy the $\tau_{e}$ constraint, differences between the simulations, while often systematic, become small and comparable to those due to differences in feedback/astrophysics/numerical limitations as described below.

Like all numerical simulations, certain limitations must be considered when interpreting the results. Limited mass and spatial resolution prohibits us from resolving the full spectrum of galaxy formation down to the lowest mass haloes that form Population III stars and thus their effects on the early Universe. Similarly, finite resolution and numerical diffusivity and viscosity suppress the amplification of magnetic fields in our simulations and reduces their strength in high-redshift galaxies where they are expected to efficiently amplify (Schober et al. 2012). Our simulation volume is far smaller than the cosmological homogeneity scale and therefore we rely on extrapolation to predict the properties of higher mass galaxies where physics might behave differently. We attempted to mitigate the effect of a small volume on the reionization history by choosing an "average" region (see Rosdahl et al. 2018); however, we must keep in mind that there may be effects that are driven by physics on scales larger than the computational volume.

Furthermore, we assumed a set of feedback models aimed at reproducing the effects of energetic processes that are not explicitly resolved by our simulations. Different SN feedback models are well known to impact galaxy properties (e.g. Rosdahl et al. 2017) as is the SED that we choose for star particles (Rosdahl et al. 2018), and radiative cooling routines applied to the gas (e.g. Gnedin \& Hollon 2012). How we model this physics can impact star formation and the escape of ionising radiation and we therefore note that certain conclusions are subject to systematic errors. Nevertheless, we have highlighted in many cases where our work is consistent with other numerical simulations presented in the literature that employ different subgrid models providing additional confidence in our results.

With these caveats in mind our main results are as follows:

- PMFs with $n_{B}>-0.562 \log _{10}\left(\frac{B_{0}}{\ln G}\right)-3.35$ as well as physical models that generate such fields pre-recombination can be ruled out due to the fact that such models result in an electron optical depth that is inconsistent with constraints from the CMB.

- Early structure formation due to PMFs can strengthen the depth of the first absorption feature in the global $21 \mathrm{~cm}$ signal at $z \sim 60-100$ due to the excess number of X-ray and $\operatorname{Ly} \alpha$ photons. This allows the spin temperature to be coupled to the kinetic temperature for a longer period of time. Hence, this regime is particularly sensitive to PMF models that cannot be ruled out by the electron optical depth.

- The studied PMF scenarios do not significantly impact the stellar mass-halo mass relation nor the intrinsic luminosity function of galaxies in the epoch of reionization, despite the modifications to the matter power spectrum and the additional pressure support from magnetic fields in the ISM.

- Galaxies in the simulations with weak PMFs (B21) have effective radii that are on average $44 \%$ larger than galaxies of similar magnitude in the simulations with strong PMFs (B11). Although this value is well within the scatter in the relation, the result is systematic. This implies that completeness fractions of galaxy surveys at faint magnitudes are lower for a Universe with a weak PMF, which will impact the determination of the high-redshift UV luminosity function.

- At UV magnitudes fainter than -12, we do not expect extrapolations of monotonic relations between galaxy size and luminosity to hold due to galaxy ageing and expansion from SN feedback. These effects should be considered in future surveys that can probe such faint magnitudes when estimating the UV luminosity function.

- The primordial magnetic fields sampled in this work do not have a strong impact on the gas density field in the post-reionization era due to photoevaporation and photoheating smoothing the density field. Thus it will prove challenging to find any impact on the postreionization Ly $\alpha$ forest. In contrast, the dark matter density field maintains memory of the modifications to the matter power spectrum and such features could impact other observables such as weak lensing.

- Strong PMFs with shallow spectral indices may impact Population III star formation and the subsequent metal enrichment that is not explicitly resolved by our simulation. However, this will only impact the reionization history at $z \gtrsim 18$ where the majority of ionising photons originate from primordial metallicity haloes.

- LyC escape fractions are $10 \%-25 \%$ higher in the simulations with strong PMFs likely due to differences in ISM properties.

- By $z=6$, the sources that are leaking half of the ionising photons have $1500 \AA$ UV magnitudes brighter than -16 and are thus well within reach of our deepest surveys. Future surveys that can reliably detect sources with $\mathrm{M}_{\mathrm{UV}}<-13$ will be able to probe the sources of reionization up to $z=12$.

In this work we have only considered the impact of primordial magnetic fields on reionization and the formation of the first galaxies. However, there are numerous other mechanisms for seeding cosmic magnetic fields based on astrophysical origins, such as the Biermann battery or in supernova remnants as discussed earlier. In future work (e.g. Attia et al. 2021), we will look to model these alternative scenarios to determine their impact on the history of reionization and the first galaxies. Our simulations are among the first to simulate the epoch of reionization with fully coupled radiationmagnetohydrodynamics and thus represent an exciting milestone for cosmological numerical simulations.

\section{ACKNOWLEDGEMENTS}

We thank the referee for their detailed revision of the manuscript. HK thanks Richard Shaw for providing us his customised version of CAMB which allowed us to create the matter initial conditions. This work was supported by the Programme National Cosmology et Galaxies (PNCG) of CNRS/INSU with INP and IN2P3, cofunded by CEA and CNES. MGH acknowledges support from the UKRI Science and Technology Facilities Council (grant numbers ST/N000927/1 and ST/S000623/1). Support by ERC Advanced Grant 320596 "The Emergence of Structure during the Epoch of reionization" is gratefully acknowledged. SMA acknowledges support by ERC Starting Grant 638707 "Black holes and their host galaxies: coevolution across cosmic time". TK was supported by the National Research Foundation of Korea (NRF-2019K2A9A1A0609137711 and NRF-2020R1C1C100707911). TG acknowledges support from the European Research Council under grant agreement ERC-stg757258 (TRIPLE). The research of AS and JD is supported by Adrian 
Beecroft and STFC. The authors gratefully acknowledge the Gauss Centre for Supercomputing e.V. (www.gauss-centre.eu) for funding this project by providing computing time on the GCS Supercomputer JUWELS (Jülich Supercomputing Centre 2019) at Jülich Supercomputing Centre (JSC). Computing time for this work was provided by the Partnership for Advanced Computing in Europe (PRACE) as part of the "First luminous objects and reionization with SPHINX (cont.)" (2019215124) project. We additionally acknowledges support and computational resources from the Common Computing Facility (CCF) of the LABEX Lyon Institute of Origins (ANR-10LABX-66).

\section{DATA AVAILABILITY}

The data underlying this article will be shared on reasonable request to the corresponding author.

\section{REFERENCES}

Attia O., Teyssier R., Katz H., Kimm T., Martin-Alvarez S., Ocvirk P., Rosdahl J., 2021, MNRAS, 504, 2346

Aubert D., Pichon C., Colombi S., 2004, MNRAS, 352, 376

Balsara D. S., 2001, Journal of Computational Physics, 174, 614

Barkana R., 2018, Nature, 555, 71

Barrow K. S. S., Robertson B. E., Ellis R. S., Nakajima K., Saxena A., Stark D. P., Tang M., 2020, ApJ, 902, L39

Basu A., Roy S., 2013, MNRAS, 433, 1675

Beck R., 2007, A\&A, 470, 539

Beck R., 2015, A\&A, 578, A93

Beck A. M., Dolag K., Lesch H., Kronberg P. P., 2013, MNRAS, 435, 3575

Behroozi P., et al., 2020, MNRAS, 499, 5702

Bera A., Datta K. K., Samui S., 2020, MNRAS, 498, 918

Bernet M. L., Miniati F., Lilly S. J., Kronberg P. P., Dessauges-Zavadsky M., 2008, Nature, 454, 302

Biermann L., 1950, Zeitschrift Naturforschung Teil A, 5, 65

Birnboim Y., 2009, ApJ, 702, L101

Blasi P., Burles S., Olinto A. V., 1999, ApJ, 514, L79

Bolton J. S., Haehnelt M. G., Warren S. J., Hewett P. C., Mortlock D. J., Venemans B. P., McMahon R. G., Simpson C., 2011, MNRAS, 416, L70

Bonvin C., Caprini C., Durrer R., 2013, Phys. Rev. D, 88, 083515

Bouwens R. J., Illingworth G. D., Oesch P. A., Maseda M., Ribeiro B., Stefanon M., Lam D., 2017a, arXiv e-prints, p. arXiv:1711.02090

Bouwens R. J., Oesch P. A., Illingworth G. D., Ellis R. S., Stefanon M., 2017b, ApJ, 843, 129

Bouwens R., et al., 2020, arXiv e-prints, p. arXiv:2009.10727

Bowman J. D., Rogers A. E. E., Monsalve R. A., Mozdzen T. J., Mahesh N., 2018, Nature, 555, 67

Brandenburg A., Subramanian K., 2005, Phys. Rep., 417, 1

Broderick A. E., Chang P., Pfrommer C., 2012, ApJ, 752, 22

Broderick A. E., Tiede P., Chang P., Lamberts A., Pfrommer C., Puchwein E., Shalaby M., Werhahn M., 2018, ApJ, 868, 87

Brooks A. M., Governato F., Booth C. M., Willman B., Gardner J. P., Wadsley J., Stinson G., Quinn T., 2007, ApJ, 655, L17

Butsky I., Zrake J., Kim J.-h., Yang H.-I., Abel T., 2017, ApJ, 843, 113

Calverley A. P., Becker G. D., Haehnelt M. G., Bolton J. S., 2011, MNRAS, 412,2543

Caprini C., Durrer R., 2002, Phys. Rev. D, 65, 023517

Caruana J., Bunker A. J., Wilkins S. M., Stanway E. R., Lorenzoni S., Jarvis M. J., Ebert H., 2014, MNRAS, 443, 2831

Chirakkara R. A., Federrath C., Trivedi P., Banerjee R., 2021, Efficient highlysubsonic turbulent dynamo and growth of primordial magnetic fields (arXiv:2101.08256)

Chluba J., Paoletti D., Finelli F., Rubiño-Martín J. A., 2015, MNRAS, 451, 2244
Chongchitnan S., Meiksin A., 2014, MNRAS, 437, 3639

Chyży K. T., Weżgowiec M., Beck R., Bomans D. J., 2011, A\&A, 529, A94

Commerçon B., Debout V., Teyssier R., 2014, A\&A, 563, A11

Crain R. A., et al., 2015, MNRAS, 450, 1937

Crutcher R. M., 2012, ARA\&A, 50, 29

D’Aloisio A., McQuinn M., Davies F. B., Furlanetto S. R., 2018, MNRAS, 473,560

Davis Leverett J., Greenstein J. L., 1951, ApJ, 114, 206

Dawoodbhoy T., et al., 2018, MNRAS, 480, 1740

Dedner A., Kemm F., Kröner D., Munz C. D., Schnitzer T., Wesenberg M., 2002, Journal of Computational Physics, 175, 645

Dekel A., Silk J., 1986, ApJ, 303, 39

Diemer B., 2018, ApJS, 239, 35

Dolag K., Stasyszyn F., 2009, MNRAS, 398, 1678

Dolag K., Kachelriess M., Ostapchenko S., Tomàs R., 2011, ApJ, 727, L4

Donnert J., Vazza F., Brüggen M., ZuHone J., 2018, Space Sci. Rev., 214, 122

Doumler T., Knebe A., 2010, MNRAS, 403, 453

Dubois Y., Teyssier R., 2008, A\&A, 482, L13

Duffin D. F., Pudritz R. E., 2009, ApJ, 706, L46

Durrive J. B., Langer M., 2015, MNRAS, 453, 345

Durrive J.-B., Tashiro H., Langer M., Sugiyama N., 2017, MNRAS, 472, 1649

Efstathiou G., 1992, MNRAS, 256, 43P

Eisenstein D. J., Hu W., 1998, ApJ, 496, 605

Eldridge J. J., Izzard R. G., Tout C. A., 2008, MNRAS, 384, 1109

Evans C. R., Hawley J. F., 1988, ApJ, 332, 659

Fan X., et al., 2006, AJ, 132, 117

Federrath C., Klessen R. S., 2012, ApJ, 761, 156

Federrath C., Schober J., Bovino S., Schleicher D. R. G., 2014, ApJ, 797, L19

Feretti L., Giovannini G., Govoni F., Murgia M., 2012, A\&ARv, 20, 54

Ferland G. J., Korista K. T., Verner D. A., Ferguson J. W., Kingdon J. B., Verner E. M., 1998, PASP, 110, 761

Fialkov A., Barkana R., 2019, MNRAS, 486, 1763

Field G. B., 1958, Proceedings of the IRE, 46, 240

Finkelstein S. L., et al., 2015, ApJ, 810, 71

Finkelstein S. L., et al., 2019, ApJ, 879, 36

Friedmann A., 1922, Zeitschrift fur Physik, 10, 377

Fromang S., Hennebelle P., Teyssier R., 2006, A\&A, 457, 371

Fukugita M., Kawasaki M., 1994, MNRAS, 269, 563

Furlanetto S. R., 2006, MNRAS, 371, 867

Furlanetto S. R., Oh S. P., Briggs F. H., 2006, Phys. Rep., 433, 181

Garaldi E., Pakmor R., Springel V., 2020, arXiv e-prints, p. arXiv:2010.09729

Gardner J. P., et al., 2006, Space Sci. Rev., 123, 485

Garel T., Blaizot J., Rosdahl J., Michel-Dansac L., Haehnelt M. G., Katz H., Kimm T., Verhamme A., 2021, MNRAS, 504, 1902

Gnedin N. Y., Abel T., 2001, New Astron., 6, 437

Gnedin N. Y., Hollon N., 2012, ApJS, 202, 13

Gnedin N. Y., Kaurov A. A., 2014, ApJ, 793, 30

Gnedin N. Y., Ferrara A., Zweibel E. G., 2000, ApJ, 539, 505

Gopal R., Sethi S. K., 2003, Journal of Astrophysics and Astronomy, 24, 51

Grasso D., Rubinstein H. R., 1995, Astroparticle Physics, 3, 95

Guillet T., Teyssier R., 2011, Journal of Computational Physics, 230, 4756

Haardt F., Madau P., 1996, ApJ, 461, 20

Hahn O., Abel T., 2011, MNRAS, 415, 2101

Harikane Y., et al., 2018, PASJ, 70, S11

Haugen N. E., Brandenburg A., Dobler W., 2004a, Phys. Rev. E, 70, 016308

Haugen N. E. L., Brandenburg A., Mee A. J., 2004b, MNRAS, 353, 947

Hennebelle P., Chabrier G., 2011, ApJ, 743, L29

Hills R., Kulkarni G., Meerburg P. D., Puchwein E., 2018, Nature, 564, E32

Hinshaw G., et al., 2013, ApJS, 208, 19

Hirata C. M., 2006, MNRAS, 367, 259

Hopkins P. F., Raives M. J., 2016, MNRAS, 455, 51

Hopkins P. F., Grudić M. Y., Wetzel A., Kereš D., Faucher-Giguère C.-A., Ma X., Murray N., Butcher N., 2020, MNRAS, 491, 3702

Hui L., Gnedin N. Y., 1997, MNRAS, 292, 27

Iliev I. T., Mellema G., Pen U. L., Merz H., Shapiro P. R., Alvarez M. A., 2006, MNRAS, 369, 1625 
Iliev I. T., Mellema G., Ahn K., Shapiro P. R., Mao Y., Pen U.-L., 2014, MNRAS, 439, 725

Ishigaki M., Kawamata R., Ouchi M., Oguri M., Shimasaku K., Ono Y., 2018, ApJ, 854, 73

Jedamzik K., Saveliev A., 2019, Phys. Rev. Lett., 123, 021301

Jedamzik K., Katalinić V., Olinto A. V., 1998, Phys. Rev. D, 57, 3264

Jülich Supercomputing Centre 2019, Journal of large-scale research facilities, 5

Kannan R., Vogelsberger M., Marinacci F., McKinnon R., Pakmor R., Springel V., 2019, MNRAS, 485, 117

Katz H., Kimm T., Sijacki D., Haehnelt M. G., 2017, MNRAS, 468, 4831

Katz H., Kimm T., Haehnelt M., Sijacki D., Rosdahl J., Blaizot J., 2018, MNRAS, 478, 4986

Katz H., Martin-Alvarez S., Devriendt J., Slyz A., Kimm T., 2019, MNRAS, 484,2620

Katz H., et al., 2020a, arXiv e-prints, p. arXiv:2005.01734

Katz H., et al., 2020b, MNRAS, 494, 2200

Kawamata R., Ishigaki M., Shimasaku K., Oguri M., Ouchi M., Tanigawa S., 2018, ApJ, 855, 4

Keating L. C., Weinberger L. H., Kulkarni G., Haehnelt M. G., Chardin J., Aubert D., 2020, MNRAS, 491, 1736

Kim E.-J., Olinto A. V., Rosner R., 1996, ApJ, 468, 28

Kimm T., Cen R., Devriendt J., Dubois Y., Slyz A., 2015, MNRAS, 451, 2900

Kimm T., Katz H., Haehnelt M., Rosdahl J., Devriendt J., Slyz A., 2017, MNRAS, 466, 4826

Körtgen B., Banerjee R., Pudritz R. E., Schmidt W., 2019, MNRAS, 489, 5004

Kroupa P., 2001, MNRAS, 322, 231

Krumholz M. R., Federrath C., 2019, Frontiers in Astronomy and Space Sciences, 6, 7

Kuhlen M., Faucher-Giguère C.-A., 2012, MNRAS, 423, 862

Kulkarni G., Keating L. C., Haehnelt M. G., Bosman S. E. I., Puchwein E., Chardin J., Aubert D., 2019, MNRAS, 485, L24

Lee K.-G., Cen R., Gott J. Richard I., Trac H., 2008, ApJ, 675, 8

Levermore C. D., 1984, J. Quant. Spectrosc. Radiative Transfer, 31, 149

Lewis A., Challinor A., Lasenby A., 2000, ApJ, 538, 473

Lewis J. S. W., et al., 2020, MNRAS, 496, 4342

Livermore R. C., Finkelstein S. L., Lotz J. M., 2017, ApJ, 835, 113

Lotz J. M., et al., 2017, ApJ, 837, 97

Ma X., Hopkins P. F., Kasen D., Quataert E., Faucher-Giguère C.-A., Kereš D., Murray N., Strom A., 2016, MNRAS, 459, 3614

Ma X., et al., 2018, MNRAS, 477, 219

Ma X., Quataert E., Wetzel A., Hopkins P. F., Faucher-Giguère C.-A., Kereš D., 2020, MNRAS, 498, 2001

Machida M. N., Tomisaka K., Matsumoto T., Inutsuka S.-i., 2008, ApJ, 677, 327

Madau P., Dickinson M., 2014, ARA\&A, 52, 415

Madau P., Haardt F., Rees M. J., 1999, ApJ, 514, 648

Marchand P., Commerçon B., Chabrier G., 2018, A\&A, 619, A37

Marinacci F., Vogelsberger M., 2016, MNRAS, 456, L69

Marinacci F., et al., 2018, MNRAS, 480, 5113

Martin-Alvarez S., Devriendt J., Slyz A., Teyssier R., 2018, MNRAS, 479, 3343

Martin-Alvarez S., Katz H., Sijacki D., Devriendt J., Slyz A., 2020a, arXiv e-prints, p. arXiv:2011.11648

Martin-Alvarez S., Slyz A., Devriendt J., Gómez-Guijarro C., 2020b, MNRAS, 495, 4475

McGreer I. D., Mesinger A., D’Odorico V., 2015, MNRAS, 447, 499

McQuinn M., Hernquist L., Zaldarriaga M., Dutta S., 2007, MNRAS, 381, 75

McQuinn M., Lidz A., Zaldarriaga M., Hernquist L., Dutta S., 2008, MNRAS, 388,1101

Meiksin A., Madau P., 2020, arXiv e-prints, p. arXiv:2006.15108

Michel-Dansac L., Blaizot J., Garel T., Verhamme A., Kimm T., Trebitsch M., 2020, A\&A, 635, A154

Mineo S., Gilfanov M., Sunyaev R., 2012, MNRAS, 419, 2095

Minoda T., Tashiro H., Takahashi T., 2019, MNRAS, 488, 2001

Mirocha J., 2014, MNRAS, 443, 1211
Mirocha J., 2020, ARES: Accelerated Reionization Era Simulations (ascl:2011.010)

Mirocha J., Harker G. J. A., Burns J. O., 2013, ApJ, 777, 118

Mittal S., Kulkarni G., 2020, MNRAS,

Miyoshi T., Kusano K., 2005, Journal of Computational Physics, 208, 315

Mo H. J., Mao S., White S. D. M., 1998, MNRAS, 295, 319

Mortlock D. J., et al., 2011, Nature, 474, 616

Moster B. P., Naab T., White S. D. M., 2018, MNRAS, 477, 1822

Muñoz J. B., Loeb A., 2018, Nature, 557, 684

Nakajima K., Ellis R. S., Robertson B. E., Tang M., Stark D. P., 2020, ApJ, 889,161

Naoz S., Yoshida N., Gnedin N. Y., 2012, ApJ, 747, 128

Naoz S., Yoshida N., Gnedin N. Y., 2013, ApJ, 763, 27

Natwariya P. K., 2020, arXiv e-prints, p. arXiv:2007.09938

Neronov A., Vovk I., 2010, Science, 328, 73

O'Shea B. W., Wise J. H., Xu H., Norman M. L., 2015, ApJ, 807, L12

Okamoto T., Gao L., Theuns T., 2008, MNRAS, 390, 920

Ono Y., et al., 2012, ApJ, 744, 83

Ota K., et al., 2008, ApJ, 677, 12

Ouchi M., et al., 2010, ApJ, 723, 869

Padoan P., Nordlund Å., 2011, ApJ, 730, 40

Pandey K. L., Sethi S. K., 2013, ApJ, 762, 15

Pandey K. L., Choudhury T. R., Sethi S. K., Ferrara A., 2015, MNRAS, 451, 1692

Pawlik A. H., Schaye J., 2008, MNRAS, 389, 651

Pawlik A. H., Schaye J., van Scherpenzeel E., 2009, MNRAS, 394, 1812

Pentericci L., et al., 2014, ApJ, 793, 113

Pillepich A., et al., 2018, MNRAS, 473, 4077

Planck Collaboration et al., 2014, A\&A, 571, A1

Planck Collaboration et al., 2016, A\&A, 594, A19

Planck Collaboration et al., 2018, arXiv e-prints, p. arXiv:1807.06209

Pontzen A., Governato F., 2012, MNRAS, 421, 3464

Powell K. G., Roe P. L., Linde T. J., Gombosi T. I., De Zeeuw D. L., 1999, Journal of Computational Physics, 154, 284

Press W. H., Schechter P., 1974, ApJ, 187, 425

Pritchard J. R., Loeb A., 2012, Reports on Progress in Physics, 75, 086901

Ratra B., 1992, ApJ, 391, L1

Read J. I., Iorio G., Agertz O., Fraternali F., 2017, MNRAS, 467, 2019

Rees M. J., 1986, MNRAS, 218, 25P

Rieder M., Teyssier R., 2017, MNRAS, 471, 2674

Robertson B. E., et al., 2013, ApJ, 768, 71

Roe P. L., 1986, Annual Review of Fluid Mechanics, 18, 337

Rosdahl J., Teyssier R., 2015, MNRAS, 449, 4380

Rosdahl J., Blaizot J., Aubert D., Stranex T., Teyssier R., 2013, MNRAS, 436, 2188

Rosdahl J., Schaye J., Dubois Y., Kimm T., Teyssier R., 2017, MNRAS, 466, 11

Rosdahl J., et al., 2018, MNRAS, 479, 994

Rosen A., Bregman J. N., 1995, ApJ, 440, 634

Saga S., Tashiro H., Yokoyama S., 2018, MNRAS, 474, L52

Sanati M., Revaz Y., Schober J., Kunze K. E., Jablonka P., 2020, arXiv e-prints, p. arXiv:2005.05401

Schenker M. A., Ellis R. S., Konidaris N. P., Stark D. P., 2014, ApJ, 795, 20

Schleicher D. R. G., Banerjee R., Klessen R. S., 2009, ApJ, 692, 236

Schmidt M., 1959, ApJ, 129, 243

Schober J., Schleicher D., Federrath C., Glover S., Klessen R. S., Banerjee R., 2012, ApJ, 754, 99

Schober J., Schleicher D. R. G., Federrath C., Bovino S., Klessen R. S., 2015, Phys. Rev. E, 92, 023010

Schroeder J., Mesinger A., Haiman Z., 2013, MNRAS, 428, 3058

Sethi S. K., Subramanian K., 2005, MNRAS, 356, 778

Sethi S. K., Subramanian K., 2009, J. Cosmology Astropart. Phys., 2009, 021

Shaw J. R., Lewis A., 2012, Phys. Rev. D, 86, 043510

Shibuya T., Ouchi M., Harikane Y., 2015, ApJS, 219, 15

Shull J. M., van Steenberg M. E., 1985, ApJ, 298, 268

Sobacchi E., Mesinger A., 2015, MNRAS, 453, 1843

Sparke L. S., 1982, ApJ, 260, 104

Stanway E. R., Eldridge J. J., Becker G. D., 2016, MNRAS, 456, 485 
Subramanian K., 2010, Astronomische Nachrichten, 331, 110

Subramanian K., 2016, Reports on Progress in Physics, 79, 076901

Subramanian K., Barrow J. D., 1998, Phys. Rev. D, 58, 083502

Subramanian K., Narasimha D., Chitre S. M., 1994, MNRAS, 271, L15

Tabatabaei F. S., Krause M., Fletcher A., Beck R., 2008, A\&A, 490, 1005

Tashiro H., Sugiyama N., 2006, MNRAS, 372, 1060

Tasinato G., 2015, J. Cosmology Astropart. Phys., 2015, 040

Tavecchio F., Ghisellini G., Bonnoli G., Foschini L., 2011, MNRAS, 414, 3566

Teyssier R., 2002, A\&A, 385, 337

Teyssier R., Fromang S., Dormy E., 2006, Journal of Computational Physics, 218, 44

Tilvi V., et al., 2014, ApJ, 794, 5

Toro E. F., 2009, Riemann Solvers and Numerical Methods for Fluid Dynamics, 3 edn. Springer

Totani T., Kawai N., Kosugi G., Aoki K., Yamada T., Iye M., Ohta K., Hattori T., 2006, PASJ, 58, 485

Tóth G., 2000, Journal of Computational Physics, 161, 605

Trac H., Cen R., Loeb A., 2008, ApJ, 689, L81

Trebitsch M., Blaizot J., Rosdahl J., Devriendt J., Slyz A., 2017, MNRAS, 470,224

Tricco T. S., Price D. J., Federrath C., 2016, MNRAS, 461, 1260

Tseliakhovich D., Hirata C., 2010, Phys. Rev. D, 82, 083520

Turner M. S., Widrow L. M., 1988, Phys. Rev. D, 37, 2743

Tweed D., Devriendt J., Blaizot J., Colombi S., Slyz A., 2009, A\&A, 506, 647

Tóth G., Roe P., 2002, Journal of Computational Physics, 180, 736

Vazza F., Brüggen M., Gheller C., Hackstein S., Wittor D., Hinz P. M., 2017, Classical and Quantum Gravity, 34, 234001

Vazza F., Brunetti G., Brüggen M., Bonafede A., 2018, MNRAS, 474, 1672

Vogelsberger M., Genel S., Sijacki D., Torrey P., Springel V., Hernquist L., 2013, MNRAS, 436, 3031

Wasserman I., 1978, ApJ, 224, 337

Wise J. H., Abel T., 2011, MNRAS, 414, 3458

Wise J. H., Turk M. J., Norman M. L., Abel T., 2012, ApJ, 745, 50

Wise J. H., Demchenko V. G., Halicek M. T., Norman M. L., Turk M. J., Abel T., Smith B. D., 2014, MNRAS, 442, 2560

Wouthuysen S. A., 1952, AJ, 57, 31

Wyithe J. S. B., Bolton J. S., 2011, MNRAS, 412, 1926

Xu H., Wise J. H., Norman M. L., Ahn K., O'Shea B. W., 2016, ApJ, 833, 84

Yoo T., Kimm T., Rosdahl J., 2020, MNRAS, 499, 5175

Zygelman B., 2005, ApJ, 622, 1356

Durovčíková D., Katz H., Bosman S. E. I., Davies F. B., Devriendt J., Slyz A., 2020, MNRAS, 493, 4256

This paper has been typeset from a $\mathrm{T}_{\mathrm{E}} \mathrm{X} / \mathrm{L} \mathrm{T}_{\mathrm{E}} \mathrm{X}$ file prepared by the author. 